\title{
Quality induced secure multiclassifier fingerprint verification using extended feature set
}

\author{
Mayank Vatsa \\ West Virginia University
}

Follow this and additional works at: https://researchrepository.wvu.edu/etd

\section{Recommended Citation}

Vatsa, Mayank, "Quality induced secure multiclassifier fingerprint verification using extended feature set" (2008). Graduate Theses, Dissertations, and Problem Reports. 4428.

https://researchrepository.wvu.edu/etd/4428

This Dissertation is protected by copyright and/or related rights. It has been brought to you by the The Research Repository @ WVU with permission from the rights-holder(s). You are free to use this Dissertation in any way that is permitted by the copyright and related rights legislation that applies to your use. For other uses you must obtain permission from the rights-holder(s) directly, unless additional rights are indicated by a Creative Commons license in the record and/ or on the work itself. This Dissertation has been accepted for inclusion in WVU Graduate Theses, Dissertations, and Problem Reports collection by an authorized administrator of The Research Repository @ WVU.

For more information, please contact researchrepository@mail.wvu.edu. 


\title{
Quality Induced Secure Multiclassifier Fingerprint Verification using Extended Feature Set
}

\author{
By \\ Mayank Vatsa \\ Dissertation submitted to the \\ College of Engineering and Mineral and Resources \\ at West Virginia University \\ in partial fulfillment of the requirements \\ for the degree of \\ Doctor of Philosophy \\ in \\ Computer Science \\ Approved by \\ Afzel Noore, $\mathrm{PhD}$, Chair \\ Larry A. Hornak, PhD \\ Arun A. Ross, PhD \\ George E. Trapp, PhD \\ Keith B. Morris, PhD \\ Morgantown, West Virginia \\ 2008
}

Keywords: Fingerprint Recognition, Level-3 Features, Information Fusion, Biometric Watermarking

Copyright 2008 Mayank Vatsa 


\begin{abstract}
Quality Induced Secure Multiclassifier Fingerprint Verification using Extended Feature Set

By

Mayank Vatsa
\end{abstract}

Automatic fingerprint verification systems use ridge flow patterns and general morphological information for broad classification, and minutiae information for verification. With the availability of high resolution fingerprint sensors, it is now feasible to capture more intricate features such as ridges, pores, permanent scars, and incipient ridges. These fine details are characterized as level-3 features and play an important role in matching and improving the verification accuracy. The main objective of this research is to develop a fast and accurate quality induced multiclassifier fingerprint verification algorithm that incorporates both level-2 and level-3 features. A quality assessment algorithm is developed that uses Redundant Discrete Wavelet Transform to extract edge, noise and smoothness information and encodes into a quality vector. The feature extraction algorithm first registers the two fingerprint images using a two-stage registration process. In the first stage, Taylor series based image transformation is used to perform coarse registration; while in the second stage, thin plate spline transformation is used for fine registration. Then, a fast Mumford-Shah curve evolution algorithm is used to extract four level-3 features namely, pores, ridge contours, dots, and incipient ridges. Gallery and probe features are matched using Mahalanobis distance measure.

Correlation analysis suggests that level- 2 and level- 3 features can be combined to improve the verification performance. Therefore, we propose five different match score fusion algorithms to combine the match scores obtained from level-2 and level-3 features. The first algorithm uses Delaunay triangulation to obtain invariant features related to level-2 and level-3 information and then combines them to generate a fused match score. The next three match score fusion algorithms utilize different techniques in information fusion namely, density based approach, classifier learning, and belief models. Experimental results show that the proposed evidence theoretic sum rule algorithm yields good performance under ideal conditions. However, if the match scores provide conflicting decisions, more sophisticated techniques are required. Belief models based fusion algorithms are ad-hoc in nature and learning algorithms require representative training dataset for correct classification. To address the limitations of these three techniques, we propose a sequential fusion algorithm which combines the learning theory and belief model with 
the statistical approach. The sequential fusion algorithm yields good verification performance at the cost of computational complexity. To optimize both verification accuracy and computational complexity, we introduce the concept of unification framework that takes into account the variability in image quality, and the characteristics of level-2 and level-3 features to select the most appropriate fusion algorithm. Experimental results on a high resolution fingerprint database show the effectiveness of the proposed algorithms.

We further propose a novel biometric watermarking algorithm to embed the level-2 and level-3 features in the face image of the same individual for increased robustness, security, and accuracy. The proposed watermarking algorithm first computes the embedding capacity in the face image using edge and corner phase congruency method. Embedding and extraction of fingerprint features is based on redundant discrete wavelet transformation. Moreover, the proposed watermarking algorithm uses adaptive user-specific watermarking parameters for improved performance. Experiments on the face-fingerprint database show that the proposed watermarking algorithm is robust to different frequency and geometric attacks, thereby securing the biometric data against tampering. 
Dedicated To My Family. 


\section{Acknowledgments}

Over the years a number of people have provided me their invaluable comments, suggestions and inspiration. As I stand at the threshold of earning my doctoral degree, I am overwhelmed when I recall all the people who have helped me get this far.

I am very grateful for the advice and support of my advisor, Dr. Afzel Noore. I have been fortunate in finding an advisor who always had an ear open for the little problems and roadblocks that unavoidably crop up in the course of performing research. His guidance was indispensable to the completion of this research and he has taught me immeasurable lessons and insights on the workings of academic research.

I acknowledge Dr. Arun Ross for invaluable suggestions, directions and comments. I feel privileged for his collaboration and I appreciate his constant encouragement. I am also thankful to my committee members Dr. Larry Hornak, Dr. Keith Morris, and Dr. George Trapp for their support and helpful suggestions. My heartfelt thanks to Dr. Brian Woerner, Dr. John Atkins, Dr. Raymond Morehead, department secretaries and systems group for their excellent support. Acknowledgment is also due to Dr. Sanjay Singh for his help and collaboration in fingerprint database preparation. National Institute of Justice is acknowledged for providing funds to carry out my research.

Furthermore, I would like to thank my friends and family members. Also, from the bottom of my heart, I thank my grandparents and parents for their sacrifices and all they have done for me. I have been blessed with their love and unwavering support throughout my life. Finally, I would like to thank my wife, Richa, for her unconditional

love and care. I appreciate her for all the innovative ideas, long discussions, and night outs. As a small token of my appreciation, I am dedicating this dissertation to my family. 


\section{Table of Contents}

List of Tables $\quad$ ix

List of Figures $\quad$ x

List of Abbreviations xiv

1 Introduction 1

1.1 Research Objectives . . . . . . . . . . . . . . . . . 8

1.2 Research contributions . . . . . . . . . . . . . . . . . . 9

2 Quality Induced Fingerprint Verification using Extended Feature Set 13

2.1 Proposed Quality Induced Fingerprint Feature Extraction and Matching Algorithm . . . . . . . . . . . . . . . . . 14

2.1.1 Local Fingerprint Image Quality Assessment . . . . . . . . . . . . 15

2.1.2 Two-Stage Non-Linear Registration Algorithm . . . . . . . . . . . 17

2.1.3 Level-3 Pore and Ridge Feature Extraction and Matching . . . . . 18

2.2 Database and Algorithms used for Evaluation . . . . . . . . . . . . 25

2.2.1 Fingerprint Database . . . . . . . . . . . . . . . 25

2.2.2 Existing Algorithms . . . . . . . . . . . . . . . . . 26

2.3 Experimental Evaluation . . . . . . . . . . . . . . . . . . 26

2.4 Summary . . . . . . . . . . . . . . . . . . . . 33

3 Integrating Image Quality in Level-2 and Level-3 Fingerprint Match Score Fusion 34

3.1 Fusion of Level-2 and Level-3 Features using Delaunay Triangulation and

Quality Incorporated Likelihood Ratio . . . . . . . . . . . . . . . . . . . 36

3.2 Evidence Theoretic Sum Rule Fusion . . . . . . . . . . . . . . . . . . . 41

3.2.1 Formulation of Evidence Theoretic Sum Rule . . . . . . . . . . . 41

3.2.2 Match Score Fusion using Evidence-Theoretic Sum Rule . . . . . 43

3.3 SVM Learning based Match Score Fusion Algorithm . . . . . . . . . . . . 44

3.3.1 Overview of $2 \nu$-SVM . . . . . . . . . . . . . . . . . . . . 44

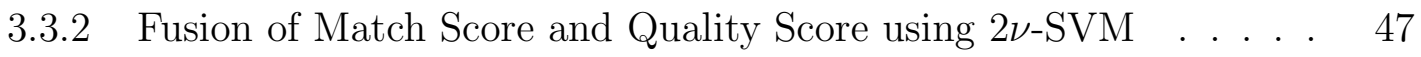

3.4 Proposed Proportional Conflict Redistribution Rule based Match Score Fusion Algorithm . . . . . . . . . . . . . . . . . . 49 
3.4.1 Overview of Belief Function Theory based Fusion Algorithms . . . 50

3.4.2 PCR5 based Biometric Match Score Fusion Algorithm . . . . . . 53

3.5 Proposed Sequential Match Score Fusion Algorithm . . . . . . . . . . . . 56

3.5.1 Sequential Match Score Fusion . . . . . . . . . . . . . . 57

3.5.2 Classification . . . . . . . . . . . . . . . . 59

3.6 Experimental Results . . . . . . . . . . . . . . . . . . . 60

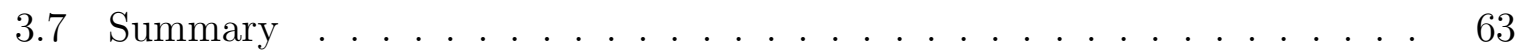

4 Unification of Fusion algorithms $\quad 69$

4.1 Framework for Unification of Fusion Algorithms . . . . . . . . . . . . . . 72

4.1.1 Rule-based Unification .................. . . 73

4.1.2 Adaptive Unification using $2 \nu$-GSVM Learning $\quad . \quad . \quad . . .73$

4.2 Experimental Results . . . . . . . . . . . . . . . . . . 76

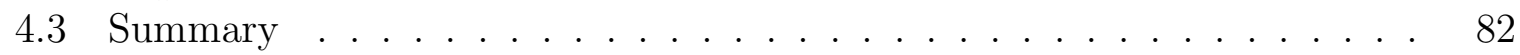

$5 \quad$ Feature based Watermarking for Secure Multimodal Biometric System 83

5.1 Proposed Biometric Watermarking Algorithm . . . . . . . . . . . . 85

5.1.1 Generating Watermark from Level-2 and Level-3 Fingerprint Features 86

5.1.2 Computing the Capacity and Locations in Face Image for Watermark Embedding . . . . . . . . . . . . . . . . . . 88

5.1.3 Embedding Fingerprint Features in Face Image . . . . . . . . . . 91

5.1.4 Extraction of Fingerprint Features from Color Face Image . . . . 92

5.1.5 Algorithmic Complexity . . . . . . . . . . . . . . 94

5.2 Verifying the Integrity of the Extracted Biometric Data . . . . . . . . . . 95

5.2.1 Performance Evaluation using Face Verification . . . . . . . . . . 96

5.2.2 Performance Evaluation using Fingerprint Verification . . . . . . . 96

5.2.3 Performance Evaluation using Multimodal Biometric . . . . . . . 97

5.2.4 Description of Databases . . . . . . . . . . . . . . . 97

5.3 Computing the Biometric Watermarking Parameters for Optimal Performance ......................... . . . . 98

5.4 Experimental Evaluation . . . . . . . . . . . . . . . . 100

5.4.1 Advantage of RDWT over DWT based Biometric Watermarking . 101

5.4.2 Performance Evaluation of Proposed Biometric Watermarking Algorithm on Attacks . . . . . . . . . . . . . . . . . . . . . . . 104

5.4.3 Effect of Embedding Watermark Data in High and Low Frequency Regions . . . . . . . . . . . . . . 110

5.5 Summary . . . . . . . . . . . . . . . . . . . . . . 110

6 Conclusions and Future Work 113

6.1 Conclusions . . . . . . . . . . . . . . . . . . 113

6.2 Future Research Directions . . . . . . . . . . . . . . . 115

$\begin{array}{ll}\text { A Dissemination of Research Results } & 117\end{array}$ 
Bibliography 


\section{List of Tables}

2.1 Comparing verification performance of the proposed quality induced level3 feature extraction and matching algorithm with existing feature extraction algorithms using fingerprints with varying number of features. Verification accuracy is computed with $\mathrm{FAR}=0.01 \%$. . . . . . . . .

3.1 Verification performance of the proposed fusion algorithms using level-2 and level-3 fingerprint features and comparing with existing match score fusion algorithms. Verification accuracy is computed at 0.01\% False Accept Rate. . . . . . . . . . . . . . . . . . . . . . . .

3.2 Verification accuracy of different SVMs and kernels at 0.01\% FAR. . . .

4.1 Comparing the verification performance of the proposed unification framework with match score fusion algorithms using level-2 and level-3 fingerprint features. Verification accuracy is computed at 0.01\% False Accept Rate. . . . . . . . . . . . . . . . . . .

5.1 Computational complexity of the proposed watermarking algorithm. . . .

5.2 Verification performance of the proposed biometric watermarking algorithm. Verification accuracy is computed at 0.01\% False Accept Rate. . .

5.3 Performance comparison of embedding level-2 and level-3 fingerprint features in high frequency regions and in low frequency regions. Face verification accuracy is computed at $0.01 \%$ FAR 


\section{List of Figures}

1.1 Example of (a) rolled, (b) slap, and (c) partial fingerprint images. . . . . 2

1.2 Fingerprint images with level-1 features: (a) arch, (b) tented arch, (c) right loop, (d) left loop, (e) double loop, and (f) whorl. . . . . . . . . . 3

1.3 Examples of (a) level-2 minutiae features and (b) level-3 features namely pores, ridge contours, dots, and incipient ridge. . . . . . . . . . . 4

1.4 Fingerprint images of an individual captured at different instances. . . . 5

1.5 Difference in image quality can cause reduced performance. . . . . . . . . 5

1.6 Gallery and probe fingerprint images can have orientation and deformation variations. . . . . . . . . . . . . . . . . .

1.7 A reduced amount of overlap between two images of the same individual can affect the verification performance. . . . . . . . . . . .

1.8 An example illustrating a case when it is difficult to capture good quality fingerprint images. . . . . . . . . . . . . . . . . .

1.9 An example illustrating a case when fingerprint is fully developed but features are not distinct. . . . . . . . . . . . . . . . . . . .

1.10 There are some features such as scars and warts that are difficult to analyze and use in fingerprint verification. . . . . . . . . . . . . . .

2.1 Illustrating the steps involved in the proposed feature extraction and matching algorithm. . . . . . . . . . . . . . . . .

2.2 Fingerprint image is partitioned into small windows for estimating the quality score. . . . . . . . . . . . . . . . . . . . 
2.3 Representative results of the two stage registration algorithm. (a) Fingerprint ridge curves (level-2) and pores (level-3) captured from two different images of the same individual, (b) registration using Taylor series [65], and (c) result of the proposed two stage registration algorithm. . . . . . . . .

2.4 Illustrating the intermediate images in the proposed feature extraction algorithm: (a) input fingerprint image, (b) edge detected image, (c) gradient image (stopping term), (d) fingerprint contour, and (e) detected pore features. . . . . . . . . . . . . . . . . . . .

2.5 Additional examples of (a) input fingerprint image, (b) stopping term $\phi$, and $(\mathrm{c})$ extracted fingerprint contour. . . . . . . . . . . . . .

2.6 Tracing of fingerprint contour and categorization of level-3 pore and ridge features. . . . . . . . . . . . . . . . . . 22

2.7 Ridge contours extracted using the proposed algorithm. . . . . . . . . . 23

$2.8 x, y$ coordinates of the dots (red) are stored as dot features. . . . . . . . 23

2.9 End points of incipient ridges (red) are marked after tracing the contour.

2.10 Matching rolled fingerprints: ROC plot for the proposed level-3 feature extraction and comparison with existing level-3 feature based algorithms $[45,58,59]$ and minutiae based algorithm. . . . . . . . . . . . .

2.11 Matching rolled to slap fingerprints: ROC plot for the proposed level3 feature extraction and comparison with existing level-3 feature based algorithms $[45,58,59]$ and minutiae based algorithm. . . . . . . .

2.12 Matching slap fingerprints: ROC plot for the proposed level-3 feature extraction and comparison with existing level-3 feature based algorithms $[45,58,59]$ and minutiae based algorithm. . . . . . . . . . . . . . .

2.13 Three sample cases: (a) when both level-2 and level-3 features based algorithms provide correct result, (b) when level-2 feature based algorithm fails to provide correct result whereas level-3 features based algorithm provides accurate decision, and (c) when both the algorithms fail to perform. . . .

3.1 Scatter plot of match scores corresponding to the level-2 and level-3 fin-

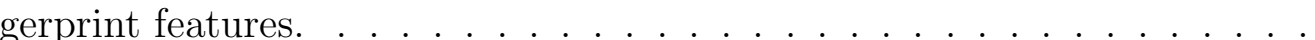

3.2 Example of (a) Voronoi diagram of fingerprint minutiae, (b) Delaunay triangulation of fingerprint minutiae, (c) minutiae triplet. . . . . . . . . 
3.3 Illustrating the steps involved in the proposed evidence theoretic sum rule. 42

3.4 Illustrating the steps involved in the $2 \nu$-support vector machine based match score fusion algorithm. . . . . . . . . . . . . . . 47

3.5 PCR rule based fusion of quality-augmented match scores obtained from level-2 and level-3 fingerprint verification algorithms. . . . . . . . . . . 54

3.6 Proposed sequential match score fusion algorithm. . . . . . . . . . . . . 57

3.7 ROC plot to evaluate the performance of the proposed evidence theoretic sum rule and comparison with the classical sum rule. . . . . . . . . . . 64

3.8 ROC plot to compare the performance of the proposed $2 \nu$-SVM match score fusion algorithm with classical SVM fusion algorithm [1]. . . . . . .

3.9 ROC plot to compare the performance of the proposed PCR5 match score fusion algorithm with other belief models. . . . . . . . . . . . . 66

3.10 ROC plot to compare the performance of the proposed sequential match score fusion algorithm, Delaunay triangulation fusion algorithm, PCR5 rule, and product of likelihood ratio fusion [67]. . . . . . . . . . . .

3.11 Sample gallery-probe pair when both level-2 and level-3 matchers accept the genuine user. . . . . . . . . . . . . . . . . . .

3.12 Sample gallery-probe pair when level-2 and level-3 matchers are in conflict but all the fusion algorithms accept the genuine user. . . . . . . . . . . . 68

3.13 Sample gallery-probe pair when level-2 matcher rejects the genuine user but level-3 matcher accepts. The proposed sequential fusion algorithm accepts the user whereas other fusion algorithm rejects. . . . . . . . . .

3.14 Sample gallery-probe pair when both level-2 and level-3 matchers rejects the genuine user. In such cases, fusion algorithms can not provide correct results. . . . . . . . . . . . . . . . . . .

4.1 General concept of the proposed unification framework. . . . . . . . . . . 71

4.2 Proposed rule-based unification framework. . . . . . . . . . . . . . 72

4.3 Proposed adaptive unification framework using $2 \nu$-GSVM. . . . . . . . 75 
4.4 ROC plot for evaluating the verification performance of the proposed rulebased and adaptive unification framework using level-2 and level-3 fingerprint features. . . . . . . . . . . . . . . . .

5.1 RDWT decomposition of a face image. Note that size of all the subbands at every level are same as the original image. . . . . . . . . . . .

5.2 Decomposition of color face image into red, green and blue channels, and corresponding phase congruency maps. . . . . . . . . . . . . . . .

5.3 Illustrating the steps involved in the proposed biometric watermarking algorithm (a) embedding process (b) extraction process. . . . . . . . .

5.4 Optimal values of $\alpha_{1}, \alpha_{2}$, and a corresponding to every face image for different individuals.

5.5 ROC for face, fingerprint, and multimodal biometrics (a) no watermarking (b) DWT watermarking (c) RDWT watermarking. . . . . . . . . . . 102

5.6 Face image with second level DWT subband and phase congruency edge map. Original face image is of size $320 \times 240$, expansive symmetric DWT subband is of size $88 \times 68$, and phase congruency edge map is subsampled to size $88 \times 68 \ldots \ldots \ldots \ldots$. . . . . . . . . . . . . . . . 103

5.7 Face images showing the effect of watermarking. . . . . . . . . . . 105

5.8 Examples of watermarked face image with different attacks (a) original image, (b) blurring, (c) filtering, (d) gamma correction, (e) JPEG compression, (f) rotation, (g) scaling, and (h) feature tampering. . . . . . 106

5.9 Examples of watermarked face image with facial feature tampering. Top row shows an example of feature removal and bottom row shows an example of feature addition. . . . . . . . . . . . . . . . 


\section{List of Abbreviations}

AFIS Automatic Fingerprint Identification System

CDEFFS Committee to Define Extended Fingerprint Feature Set

C-SVM Classical Support Vector Machine

DST Dempster Shafer Theory

DSmT Dezert Smarandache Theory

DWT Discrete Wavelet Transform

EER Equal Error Rate

FAR False Accept Rate

FBI-IAFIS Federal Bureau of Investigation - Integrated Automatic

Fingerprint Identification System

FLDA Fisher Linear Discriminant Analysis

FRR False Reject Rate

GAR Genuine Accept Rate

IDWT Inverse Discrete Wavelet Transform

IRDWT Inverse Redundant Discrete Wavelet Transform

LBP Local Binary Patterns

LFA Local Feature Analysis

PCA Principle Component Analysis

PCR Proportional Conflict Redistribution

RDWT Redundant Discrete Wavelet Transform

ROC Receiver Operating Characteristics

RDWT Redundant Discrete Wavelet Transform

SVM Support Vector Machine

TBM Theory of Belief Models

TPS Thin Plate Spline

$\nu$-SVM $\quad \nu$-Support Vector Machine

$2 \nu$-SVM Dual $\nu$-Support Vector Machine

$2 \nu$-GSVM Dual $\nu$-Granular Support Vector Machine 


\section{Chapter 1}

\section{Introduction}

Fingerprints are considered reliable to identify individuals and are used in both biometric and forensic applications. In biometrics applications, it is used for physical access control, border security, watch list, background check, and National ID System whereas in forensics applications, it is used for latent fingerprint matching for crime scene investigation and to apprehend criminals and terrorists. The use of fingerprints for establishing the identity was started in the 16th century and thereafter several studies were performed for anatomical formation, classification, recognition, categorizing features, individuality of fingerprints, and many others $[6,18,23,35,37,62,64,66,68,69,79,80,95,96]$. Over the last two decades, research in fingerprint recognition has seen tremendous growth. Several automated systems have been developed and used for civil, military and forensic applications. FBI-AFIS, US border security, and EU passport/ID system are few examples of large scale applications of fingerprint biometrics.

In fingerprint recognition, there are two fundamental steps: (i) acquisition and (ii) matching. Fingerprint images are acquired either using ink-paper based offline acquisition approach or using automated sensors such as optical and solid state sensors based livescan acquisition approach. The acquired fingerprint images provide the impression of ridges and furrows that are used in matching. Further, the acquired image may be rolled, 


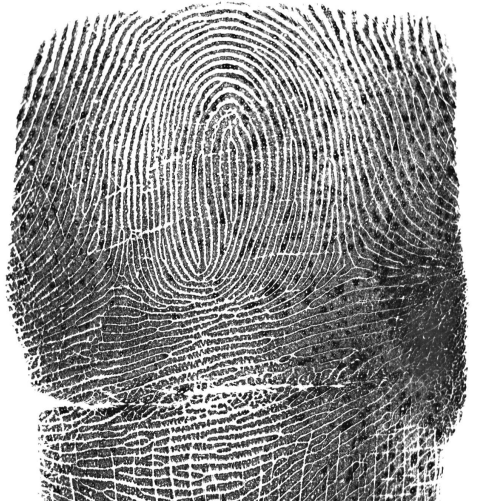

(a)

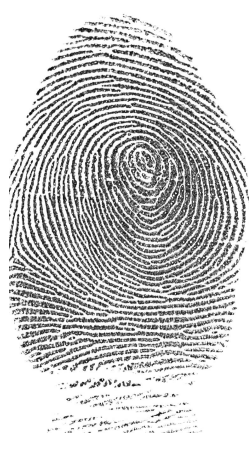

(b)

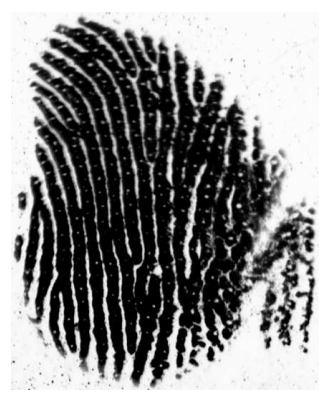

(c)

Figure 1.1: Example of (a) rolled, (b) slap, and (c) partial fingerprint images.

slap or partial. Figure 1.1 shows examples of rolled, slap, and partial images. Fingerprint matching is the process in which a probe image is matched with the gallery image(s) for classification, verification, or identification depending on the application. In classification, a fingerprint is classified into a global category such as arch, loop, and whorl using the global patterns and morphological information. In verification (1: 1 matching), the identity of a probe image is verified using details such as minutiae and pores. Finally, fingerprint identification establishes the identity of a probe image against a gallery of fingerprint images, i.e. $1: N$ matching.

In general, fingerprint features are characterized into three levels:

- Level-1 Features: Macro details such as pattern type, ridge flow and morphological features are termed as level-1 features. Figure 1.2 shows examples of level-1 features such as arch, tented arch, right loop, left loop, double loop, and whorl.

- Level-2 Features: Galton features are referred to as level-2 features. These features are ridge ending and ridge bifurcation as shown in Figure 1.3a. 


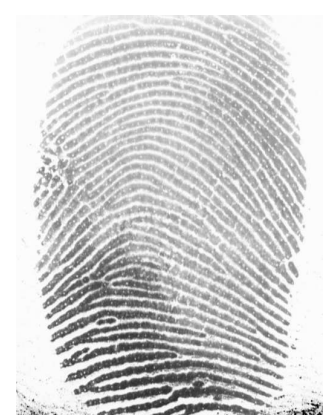

(a)

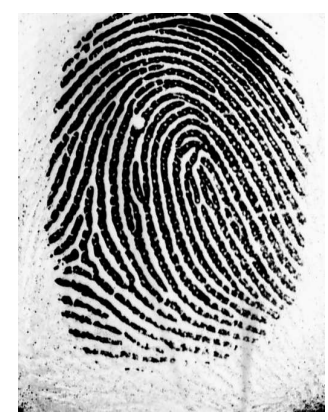

(d)

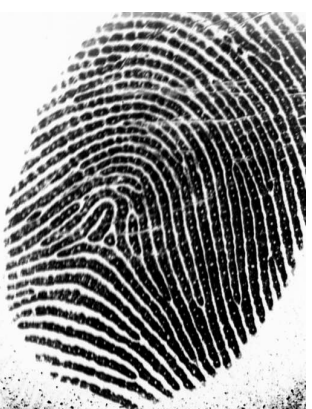

(b)

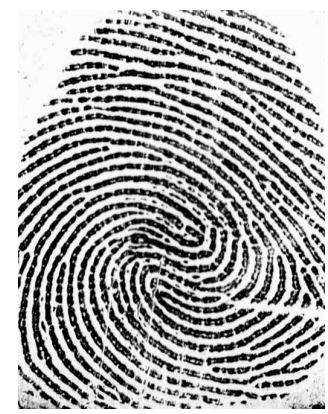

(e)

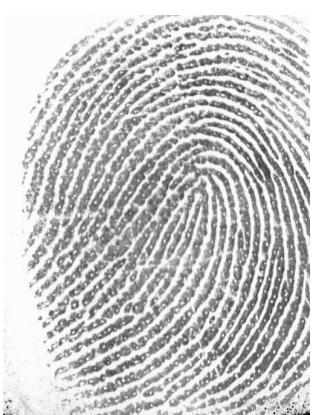

(c)

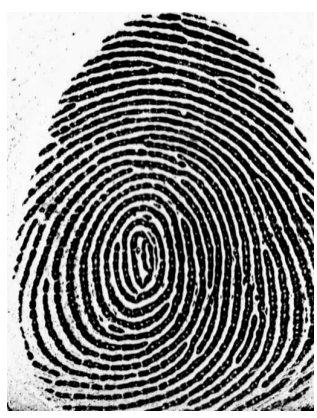

(f)

Figure 1.2: Fingerprint images with level-1 features: (a) arch, (b) tented arch, (c) right loop, (d) left loop, (e) double loop, and (f) whorl.

- Level-3 Features: ANSI/NIST Committee to Define an Extended Fingerprint Feature Set (CDEFFS) [113] has defined micro features such as pores, ridge contours, dots, and incipient ridges as level-3 features. Figure 1.3b shows an example of level-3 features in a fingerprint image.

In general, automatic fingerprint verification algorithms use level-1 features for broad classification and level-2 minutia features for verification [64]. Forensic experts, on the other hand, use manually marked level-3 features along with level-1 and level2 features for matching latent and partial fingerprints. Unlike level-2 features, level-3 features have not been extensively investigated by the research community. Very few researchers have proposed algorithms for level-3 based fingerprint verification [19, 45, $58,59,65,74]$. These algorithms perform well with good quality images but have high 


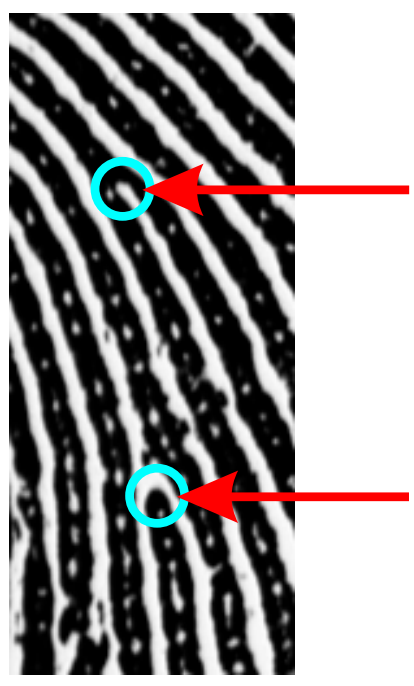

(a)

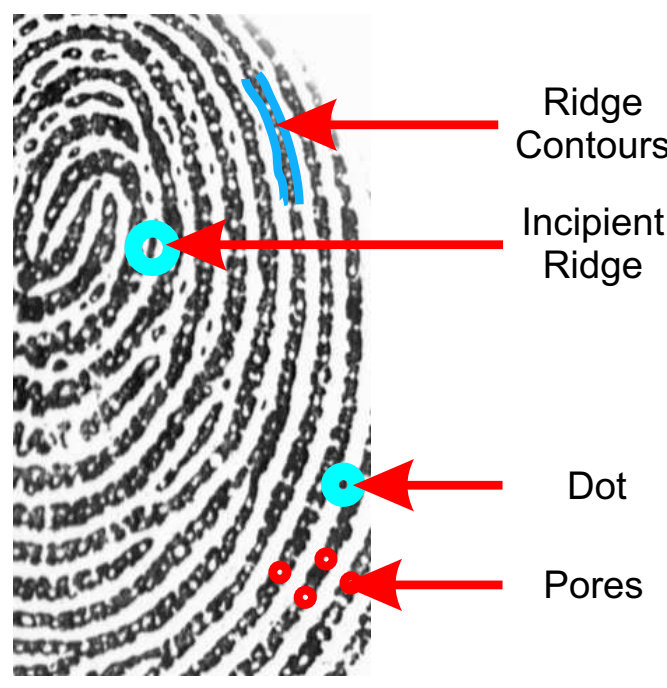

(b)

Figure 1.3: Examples of (a) level-2 minutiae features and (b) level-3 features namely pores, ridge contours, dots, and incipient ridge.

computational complexity.

Sensor noise, poor quality, and partial capture may affect the performance of fingerprint verification algorithms. For example, Figure 1.4 shows an example in which fingerprint images of an individual are captured at five different instances. This example illustrates that the interaction between an individual and the sensor can cause variations in image quality and biometric features. Specifically, gallery and probe images may have differences due to image quality (Figure 1.5), orientation and deformation (Figure 1.6), and reduced amount of overlap (Figure 1.7). Further, there are cases when it is very difficult to capture good quality images. Figure 1.8 shows images of an individual that are captured at different sessions and the image quality does not improve even with applying more pressure. Also, Figure 1.9 shows a fully developed fingerprint pattern where accurate feature extraction is not viable. Finally, scars and warts also affect the feature extraction process as they are difficult to analyze (Figure 1.10). 


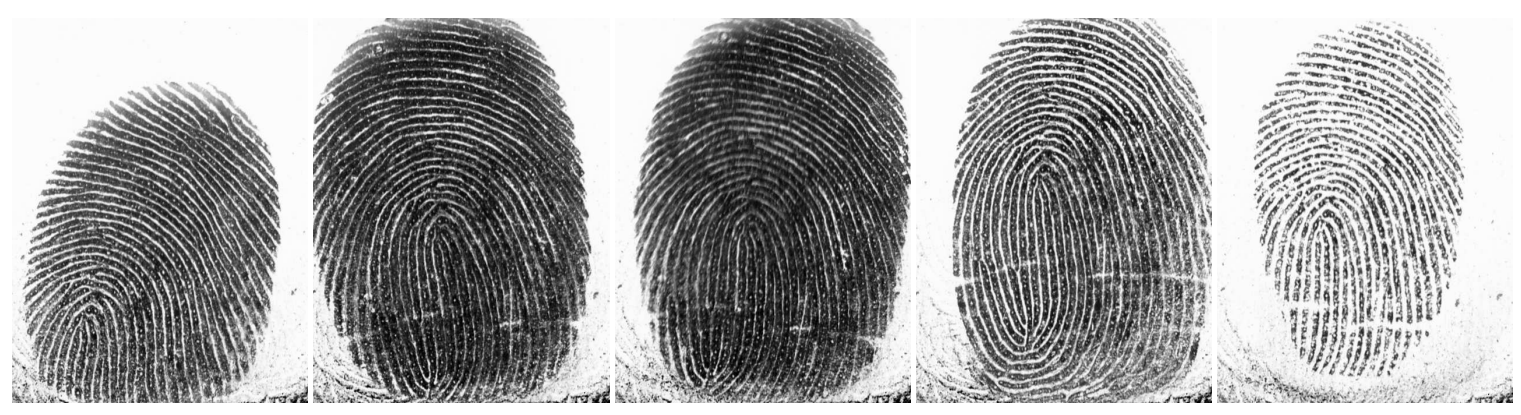

Figure 1.4: Fingerprint images of an individual captured at different instances.

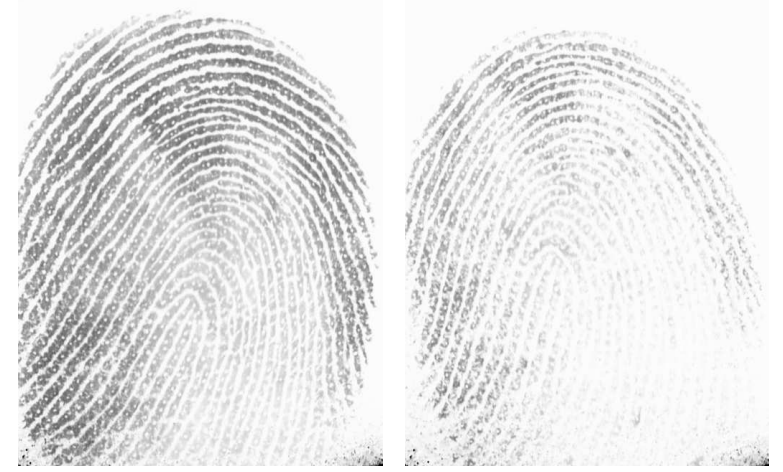

Figure 1.5: Difference in image quality can cause reduced performance.

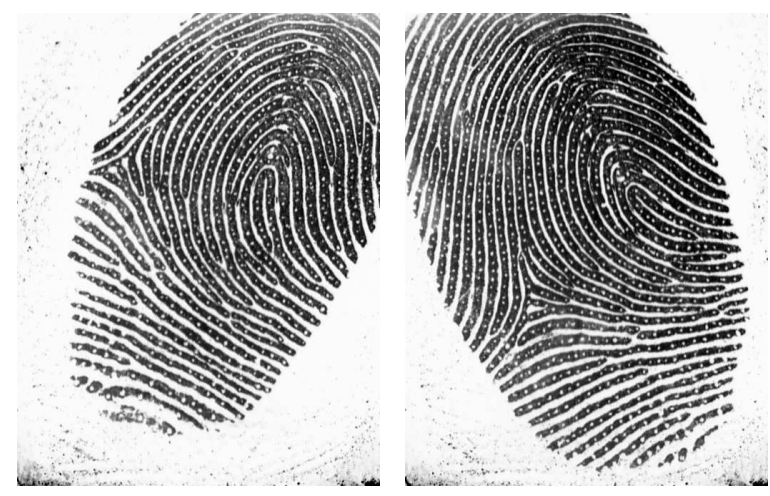

Figure 1.6: Gallery and probe fingerprint images can have orientation and deformation variations. 


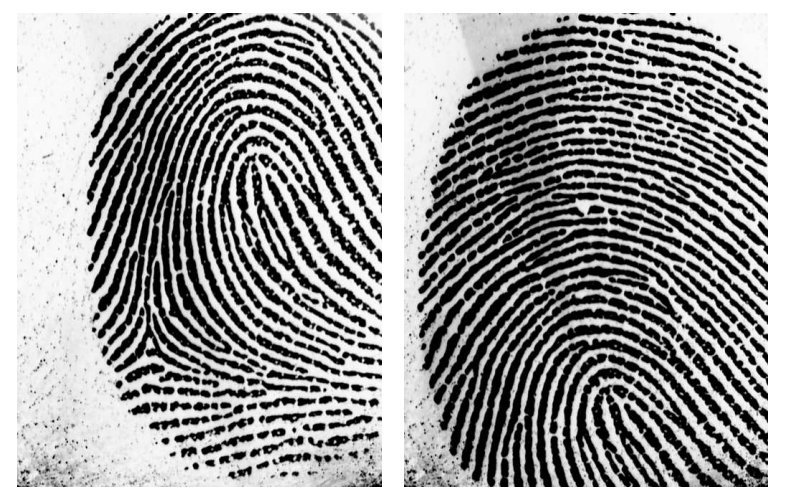

Figure 1.7: A reduced amount of overlap between two images of the same individual can affect the verification performance.

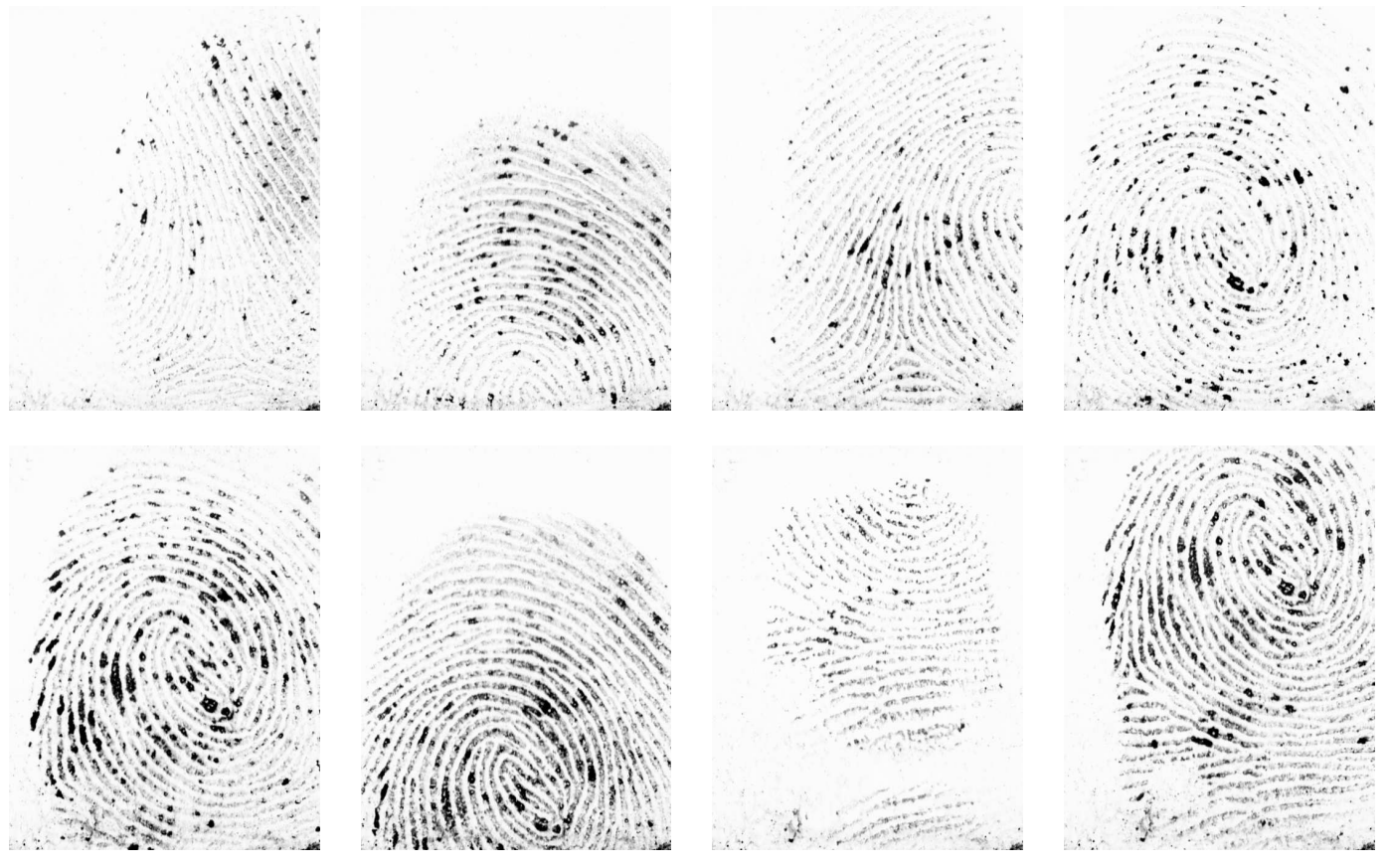

Figure 1.8: An example illustrating a case when it is difficult to capture good quality fingerprint images. 

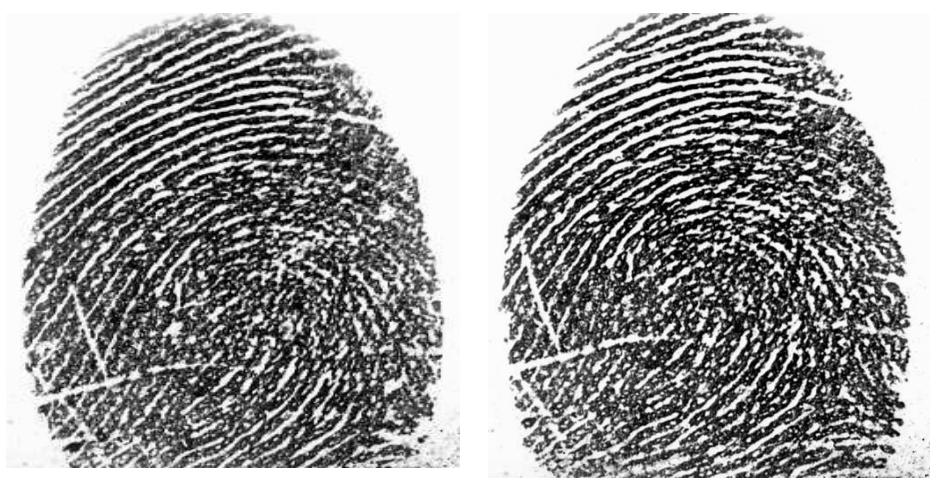

Figure 1.9: An example illustrating a case when fingerprint is fully developed but features are not distinct.
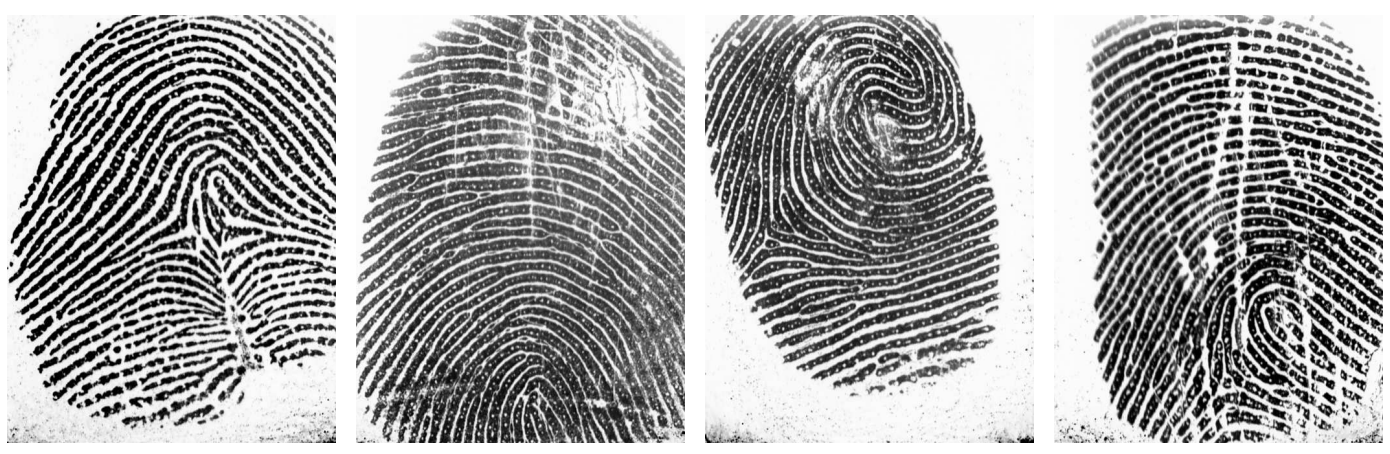

Figure 1.10: There are some features such as scars and warts that are difficult to analyze and use in fingerprint verification. 
Researchers have proposed algorithms to address challenges due to image quality, orientation and deformation [64]. However, image quality enhancement and deformation correction algorithms do not necessarily preserve both level-2 and level-3 features. It is also suggested that fusion of level-2 and level-3 information can improve the verification performance [45], nonetheless a thorough investigation is required. Therefore, currently the main challenge in level-3 fingerprint verification is to design a fast feature extraction and matching algorithm, incorporate ancillary information such as image quality and combine level-3 features with level-2 features using fusion techniques for improved performance.

In addition, the security of biometric data is of paramount importance and must be protected from external attacks and tampering [53]. Ratha et al. [72] characterized common attacks in biometric systems as coercive attack, impersonation attack, replay attack, and attacks on feature extractor, template database, matcher, and matching results. Attacks can alter the contents of biometric images or templates and can degrade the performance of a biometric system. It is therefore required to protect the biometric templates of individuals at all times. On the same note, fingerprint features, both level-2 and level-3, need to be secured from external tampering. One approach for securing fingerprint features is to embed these features into the individual's face image using watermarking.

\section{$1.1 \quad$ Research Objectives}

This research focuses on designing quality induced algorithms for level-3 fingerprint feature extraction, fusion techniques for efficiently combining level-2 and level-3 features, and securing the fingerprint features using watermarking techniques. The research ob- 
jectives are:

- Design a quality induced algorithm for level-3 feature extraction from fingerprint images.

- Design fusion algorithms using learning and belief model based techniques to efficiently combine level-2 and level-3 features at the match score level.

- Design a unification framework that includes multiple fusion algorithms to optimize verification accuracy and computational time required for information fusion.

- Design a watermarking technique with fingerprint features as the watermark and face as the cover image.

\subsection{Research contributions}

This section provides an overview of the algorithms designed to accomplish the above mentioned research objectives.

\section{Quality Induced Fingerprint Verification using Extended Fingerprint Feature Set}

To address the challenges in level-3 feature extraction and matching, we developed a novel algorithm for quality induced fast fingerprint verification using level-2 and level-3 features. The algorithm first applies Redundant Discrete Wavelet Transform to encode the degree of irregularity present in the local regions. The gallery and probe fingerprint images are registered using a two-stage registration process. Next, the curve evolution technique [83] is used to design a fast level-3 feature extraction algorithm for extracting pores, ridge contours, dots, and incipient ridges. Experiments are conducted using a high 
resolution fingerprint database of more than 710 classes and the performance is compared with existing algorithms.

\section{Integrating Image Quality in Level-2 and Level-3 Fingerprint Match Score Fusion}

To improve the verification performance of fingerprint recognition, we have designed five match score fusion algorithms, (1) Delaunay triangulation based fusion algorithm, (2) Evidence theoretic sum rule, (3) Support Vector Machine fusion algorithm, (4) Proportional Conflict Redistribution rule based fusion algorithm, and (5) Sequential match score fusion algorithm. Evidence theoretic sum rule is based on basic probability assignments computed from the match scores. Compared to traditional sum rule [78], it also incorporates prior evidences such as image quality and verification accuracy computed using the training dataset. Support Vector Machine fusion algorithm is a classification based fusion algorithm that incorporates $2 \nu$-support vector machine $[22,107]$ to fuse the quality induced match scores of level-2 and level-3 fingerprint features. On the other hand, PCR based fusion algorithm efficiently combines the imprecise and incomplete information by applying the Proportional Conflict Redistribution rule $[26,89]$. The experimental analysis of these fusion algorithms show that the evidence theoretic sum rule suffers due to errors in density estimation, PCR fusion algorithm is computationally expensive and ad-hoc in nature, whereas classifier-based fusion schemes are too rigid and often lead to a transductive type of learning that cannot handle conflicting match scores effectively. To address these limitations, we propose a sequential fusion algorithm that bridges the gap between these three types of fusion schemes. The density estimation and likelihood ratio aspect of the algorithm makes it robust to uncertainties in the component matchers; the use of belief model makes it robust to conflicting cases, and the use of 
Support Vector Machine ensures that the algorithm is less prone to over-fitting thereby permitting it to handle non-linear biometric information.

\section{Unification of Fusion Algorithms}

We have proposed an unification framework that reconciles multiple fusion algorithms to efficiently address both accuracy and time complexity of multiclassifier fingerprint recognition. It is suggested that unification or reconciliation of multiple fusion algorithms should satisfy most of the application requirements and yield better recognition performance. The proposed unification framework includes a collection of fusion algorithms and uses the evidences obtained from the input biometric probe data to dynamically select the optimal fusion algorithm. The selected fusion algorithm is then used for fusion and decision making. The evidences which serve as input to the unification framework are image quality, match scores, and verification prior (precision of true verification score computed from the matcher). Specifically, we have introduced two novel unification frameworks to combine match score fusion algorithms: (1) Rule-based unification, (2) Learning-based unification. The first unification framework uses a fixed rule-based strategy to unify fusion algorithms. We further extend the fixed rule-based unification strategy by incorporating an intelligent learning technique for adaptive unification. Experimental results on fingerprint database show that the unification framework improves the performance both in terms of accuracy and time.

\section{Feature based Watermarking for Secure Multimodal Biometric System}

In this research, we propose a novel biometric watermarking algorithm using RDWT in which color face image is used as the host image and level-2 and level-3 fingerprint features extracted from the fingerprint image are used as the watermark. Face and finger- 
print are chosen for watermarking because of the widespread use of face and fingerprint verification in applications such as the US-VISIT. There are several applications where either face or fingerprint or both are used to authenticate an individual. The proposed watermarking algorithm first computes the embedding capacity in the face image using edge and corner phase congruency method [57]. Embedding and extraction of fingerprint features is based on redundant discrete wavelet transformation [25]. The performance of the proposed watermarking algorithm is validated using face, fingerprint and multimodal verification algorithms. We observe that the proposed watermark embedding and extraction algorithm does not affect the quality of the original face image or the recognition performance. In addition, the proposed algorithm is robust and resilient to common attacks. 


\section{Chapter 2}

\section{Quality Induced Fingerprint}

\section{Verification using Extended Feature}

\section{Set}

With the advancement in sensor technology, high resolution fingerprint images $(\geq$ 1000 dpi) provide multiple levels of features that can be used for identification. Existing automatic fingerprint identification systems use level-1 features such as core, delta, and loops for classification and level-2 minutia features for recognition [64]. Forensic experts, on the other hand, use level-3 features such as pores, ridges, dots, and scars along with level-1 and level-2 features for matching latent fingerprints. It is an important research problem to identify unique and discriminating level-3 features and combine them with level-2 features for automatic recognition. ANSI/NIST CDEFFS [113] and federal agencies are also investigating fingerprint features that can be used for recognition.

Researchers have proposed several algorithms for level-2 fingerprint recognition [64]. However, limited research has been performed for level-3 feature extraction and matching $[45,58,59,65,74]$. The recognition performance of these algorithms are good but 
have certain limitations. The algorithm proposed in [74] requires manual intervention for fingerprint alignment whereas the algorithm proposed in $[58,59]$ requires very high resolution ( $\geq 2000$ ppi) images. Meenen et al. [65] proposed a pore extraction algorithm that suffers due to elastic distortion and misclassification of pore features. Jain et al. [45] proposed an automated algorithm that utilizes minutiae information for alignment. Level-3 features are then extracted and matching is performed using iterative closest point algorithm. However, level-3 feature extraction and matching algorithm is computationally expensive.

To address these challenges, we develop a quality induced fast fingerprint verification algorithm that uses level-3 features. The algorithm first computes an image quality vector using Redundant Discrete Wavelet Transform that encodes the degree of irregularity present in the local regions. Next, the gallery and probe fingerprint images are registered using a two-stage registration process that utilizes Taylor series transformation and thin plate splines. A fast level-3 feature extraction algorithm using the MumfordShah curve evolution technique is proposed for extracting four level-3 features namely pores, ridge contours, dots, and incipient ridges. Finally, a composite feature vector is generated for matching by incorporating local image quality score. Experimental results on a high resolution fingerprint database that contains both rolled and slap fingerprint images show the effectiveness of the proposed algorithms.

\subsection{Proposed Quality Induced Fingerprint Feature Extraction and Matching Algorithm}

Figure 2.1 illustrates the steps involved in the proposed feature extraction and matching algorithm. The proposed algorithm starts with computing the fingerprint image 


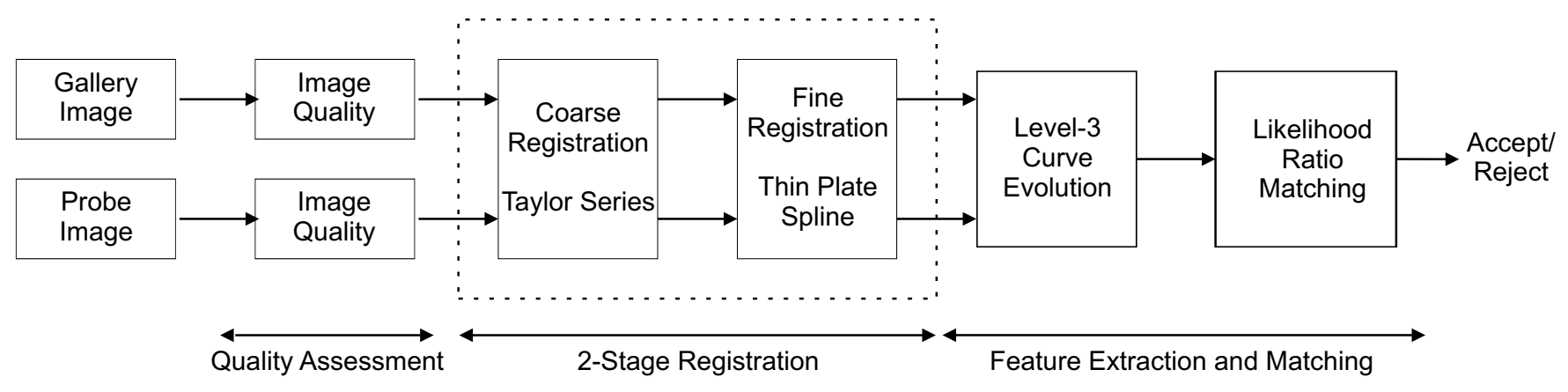

Figure 2.1: Illustrating the steps involved in the proposed feature extraction and matching algorithm.

quality score followed by a two-stage registration process using level-2 features. Level-2 minutiae are extracted from a fingerprint image using the ridge tracing algorithm [55] and matched using a dynamic bounding box based matching algorithm [48]. The details of the minutiae extraction and matching algorithms can be found in $[48,55]$. Next, we extract the level-3 features using curve evolution approach and match them using likelihood ratio based measure. This section presents each of these steps in detail.

\subsubsection{Local Fingerprint Image Quality Assessment}

In general, the performance of a fingerprint recognition algorithm depends on the quality of the probe image. Image quality can vary locally in a fingerprint image and factors such as sensor noise, pressure, and wetness can affect the quality. Therefore, it is important to compute the image quality locally and encode the edge information, smoothness and noise present in the fingerprint image.

Let $F$ be a high resolution fingerprint image. As shown in Figure 2.2, $F$ is divided into windows of size $n \times m$. For the $k^{\text {th }}$ block, $F^{k}$, RDWT decomposition is computed for $j=1, \ldots, l$ levels. 


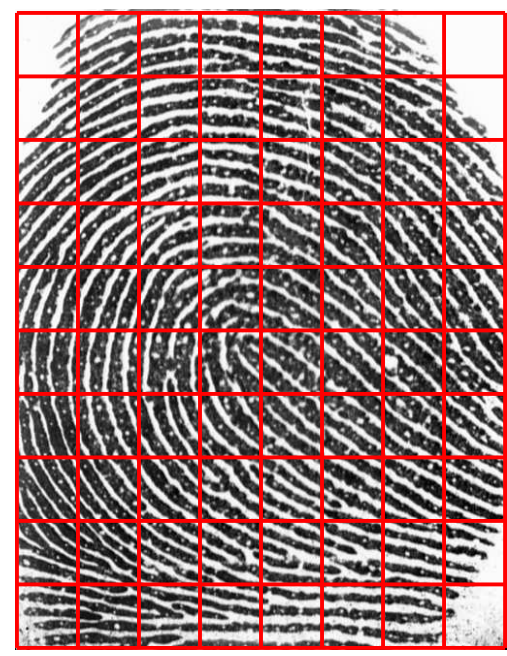

Figure 2.2: Fingerprint image is partitioned into small windows for estimating the quality score.

$$
\left[F_{A j}^{k}, F_{H j}^{k}, F_{V j}^{k}, F_{D j}^{k}\right]=R D W T\left(F^{k}\right)
$$

where, $i=A, H, V, D$ represents the approximation, horizontal, vertical, and diagonal subbands. For each block, the quality score is computed using Equation 2.2.

$$
q^{k}=\frac{a_{A}^{k} b_{A}^{k}+a_{H}^{k} b_{H}^{k}+a_{V}^{k} b_{V}^{k}+a_{D}^{k} b_{D}^{k}}{b_{A}^{k}+b_{H}^{k}+b_{V}^{k}+b_{D}^{k}}
$$

where

$$
a_{i}^{k}=\sum_{j=1}^{l} \ln \sqrt{\left(\frac{\mu_{i j}^{k}-\sum_{j=1}^{l} \sum_{x, y=1}^{n, m} F_{i j}^{k}(x, y)}{\sigma_{i j}^{k}}\right)^{2} / n m}
$$

and

$$
b_{i}^{k}=\sum_{j=1}^{l} \ln \sqrt{\left(\frac{1}{1+\sum_{x, y=1}^{n, m} \nabla F_{i j}^{k}(x, y)}\right)^{2} / n m}
$$

Here, $\mu_{i j}^{k}$ and $\sigma_{i j}^{k}$ are the mean and standard deviation of the RDWT coefficients of the $i^{\text {th }}$ subband and the $j^{\text {th }}$ level respectively, and $\nabla$ denotes the gradient operator. Finally, 
the quality score, $q^{k}$, is normalized in the range of $[0,1]$ using min-max normalization.

Multilevel RDWT decomposition provides the per-subband noise relationship and the spatial and frequency information which are useful in computing the edge and noise information [33]. The algorithm also performs error normalization by incorporating the weight factor, $b_{i}$, and encoding the degree of irregularity in the local regions. The proposed local quality assessment algorithm yields a quality score for every local region. Thus, a quality score vector, $\mathbf{q}$, is computed for the complete fingerprint image and is used during recognition.

\subsubsection{Two-Stage Non-Linear Registration Algorithm}

Fingerprint images are subjected to non-linear deformation due to varying amount of pressure applied by a user at different instances of image capture. These deformations affect the unique spatial distribution and characteristics of fingerprint features. To address these non-linearities and accurately register the probe fingerprint image with respect to the gallery fingerprint image, a two-stage non-linear registration algorithm is proposed. The registration process uses two existing non-linear registration algorithms: Taylor series transformation [65] and thin plate spline based ridge curve correspondence [75]. Taylor series based algorithm performs an image transformation that takes fingerprint images along with their minutiae coordinates as input and computes the registration parameter using Taylor series. We use this algorithm to coarsely register the gallery and probe images in the first stage. In the next stage, we use the more detailed and accurate

thin plate spline based ridge curve correspondence [75] algorithm for fine registration of fingerprint images. Thin plate spline algorithm is used at the second stage because it requires coarsely registered features as input. Fingerprint images contain a large number of level-3 features that increase the complexity of the thin plate spline algorithm. We 


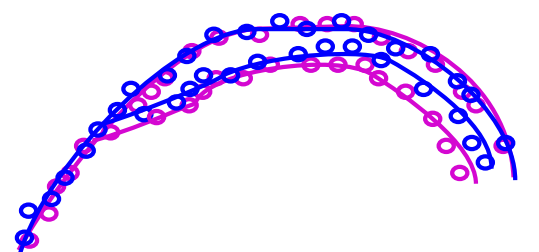

(a)

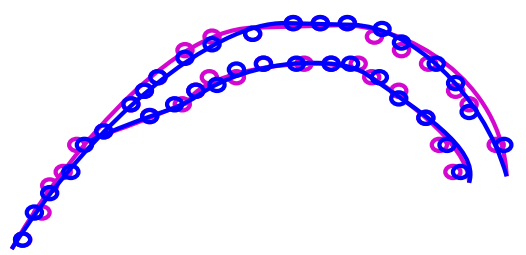

(b)

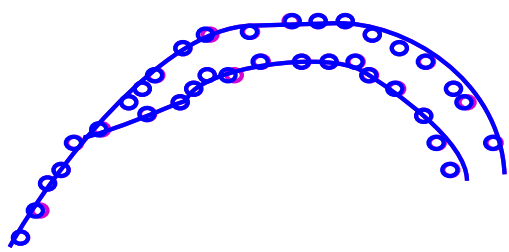

(c)

Figure 2.3: Representative results of the two stage registration algorithm. (a) Fingerprint ridge curves (level-2) and pores (level-3) captured from two different images of the same individual, (b) registration using Taylor series [65], and (c) result of the proposed two stage registration algorithm.

therefore use only level-2 features to accurately register the gallery and probe images. The non-linear two stage approach thus registers the gallery and probe fingerprint images with respect to rotation, scaling, translation, feature positions, and local deformation. Figure 2.3 illustrates the results obtained with the proposed two stage registration algorithm. Note that Figure 2.3(c) shows that a segment of ridge curves and pores obtained from both the gallery and probe images are perfectly registered and appear superimposed.

\subsubsection{Level-3 Pore and Ridge Feature Extraction and Matching}

The level-3 feature extraction algorithm employs level-set based curve evolution [83] which begins with the energy functional [17, 102]

$$
E(C)=\int_{0}^{1}\left(E_{\text {in }} C(q)+E_{\text {out }} C(q)\right) d q,
$$

where, $C(q):[0,1] \rightarrow R^{2}$ is a planar curve that can be applied on the image $F:[0, x] \times$ $[0, y] \rightarrow R^{+}$to detect feature boundaries. $E_{\text {in }}$ and $E_{\text {out }}$ are the energy functionals inside and outside the curve respectively. The energy functional can be further decomposed into Equation 2.6 using the input image $F$. 


$$
\begin{gathered}
E\left(C, C_{1}, C_{2}\right)=\alpha \iint_{\Omega} \phi|| \bar{C}^{\prime}|| d x d y+\beta \iint_{\text {in }(C)}\left|F-C_{1}\right|^{2} \\
d x d y+\lambda \iint_{\text {out }(C)}\left|F-C_{2}\right|^{2} d x d y
\end{gathered}
$$

where, $\alpha, \beta$ and $\lambda$ are positive constants such that $\alpha+\beta+\lambda=1$ and $\alpha<\beta \leq \lambda$, $\Omega$ represents the image domain, $\phi$ is the stopping term, $\bar{C}$ is the evolution curve, i.e., $\bar{C}=\{(x, y): \bar{\psi}(x, y)=0\}$, and $C_{1}$ and $C_{2}$ are the average pixel values inside and outside the curve respectively. Further, parameterizing the energy equation by an artificial time $t \geq 0$ and deducing the associated Euler-Lagrange equation leads to the following active contour model,

$$
\bar{\psi}_{t}^{\prime}=\alpha \phi\left(\bar{\nu}+\epsilon_{k}\right)|\nabla \bar{\psi}|+\nabla \phi \nabla \bar{\psi}+\beta \delta\left(F-C_{1}\right)^{2}+\lambda \delta \bar{\psi}\left(F-C_{2}\right)^{2}
$$

where $\bar{\nu}$ is the advection term and $\epsilon_{k}$ is the curvature based smoothing term. $\nabla$ is the gradient and $\delta=0.5 /\left(\pi\left(x^{2}+0.25\right)\right)$. The stopping term $\phi$ is set to

$$
\phi=\frac{1}{1+(|\nabla F|)^{2}}
$$

The basic concept of feature boundary extraction using level-set curve evolution is to initialize the contour $\bar{\psi}$ over the image $F$ and the contour $\bar{\psi}$ converges to the boundaries based on the stopping term $\phi$. The curve evolution efficiently segments the contours present in the image irrespective of the quality of the image. However, this procedure is time consuming and depends on the initial $\bar{\psi}$. On a 1000 dpi fingerprint image, if the initial contour is initialized as a grid, level-set curve evolution algorithms require around 4-30 seconds to find the feature boundaries. For real world biometric systems, such high computational complexity is not pragmatic. To address this issue, we 


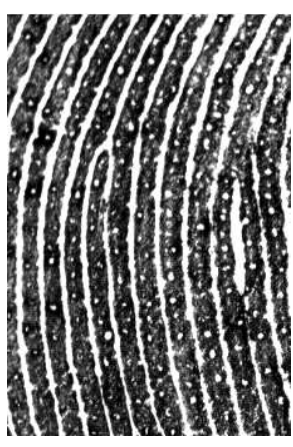

(a)

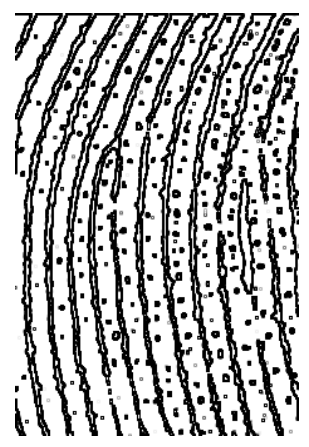

(b)

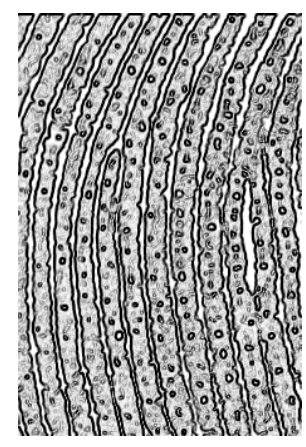

(c)

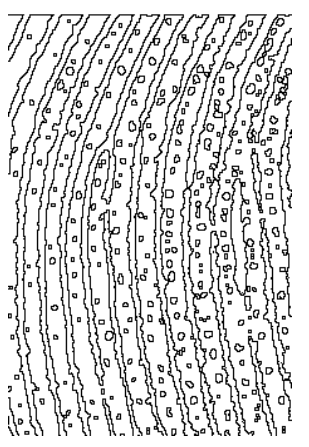

(d)

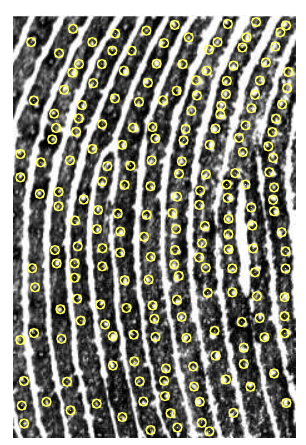

(e)

Figure 2.4: Illustrating the intermediate images in the proposed feature extraction algorithm: (a) input fingerprint image, (b) edge detected image, (c) gradient image (stopping term), (d) fingerprint contour, and (e) detected pore features.

propose a scheme that utilizes the scale multiplication based Canny edge detection [8] for finding the initial $\bar{\psi}$ and then applies a two-cycle fast curve evolution algorithm with smoothness regularization [83]. The contour extraction algorithm is as follows:

Step 1: Scale multiplication based edge detection algorithm (that multiples filter response at adjacent scales to enhance the edge structure and detect the edges as the local maxima) [8] is applied on the input fingerprint image. The output of edge detection is a binary image in which the edges are black and non-edge regions are white. The detected regions are very close to the exact feature boundaries but may also have spurious edges due to noise. To remove these spurious edges, every non-connecting edge of 1-2 pixels are removed (morphological operation).

Step 2: In the next step, the detected edges are used as the initial contour $\bar{\psi}$ and the twocycle curve evolution algorithm [83] is applied in which the first cycle is data dependent and the second cycle is Gaussian filter band smoothing. Since the initial contour is very close to the exact feature boundaries, using the stopping term $\phi$ the curve evolution algorithm converges to the feature boundaries after only few iterations. Figures 2.4(a)- 

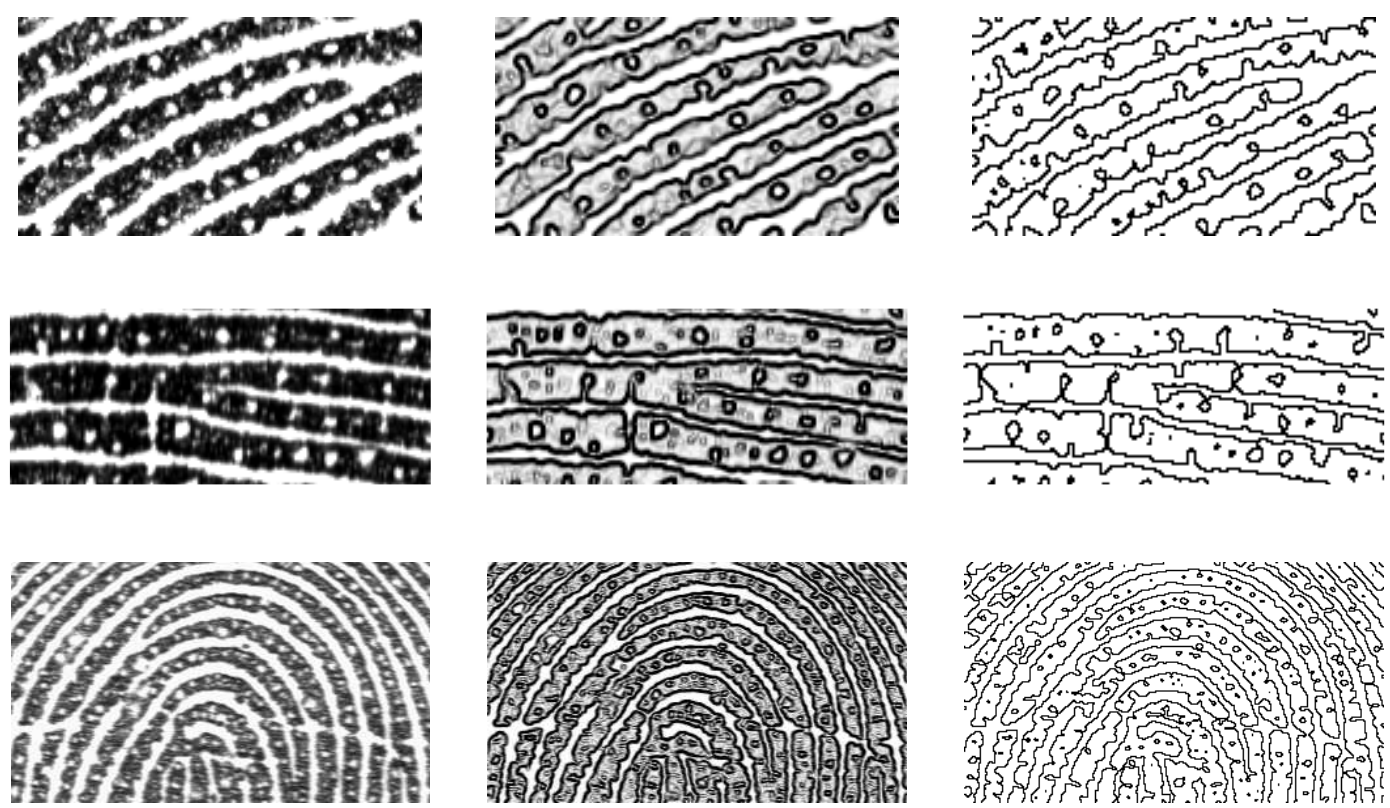

(a)

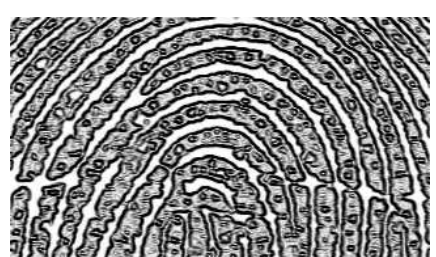

(b)

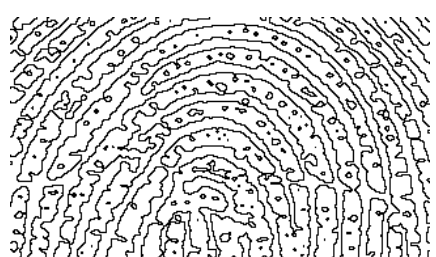

(c)

Figure 2.5: Additional examples of (a) input fingerprint image, (b) stopping term $\phi$, and (c) extracted fingerprint contour.

(d) show an example of the edge detection and contour extraction procedure. Additional examples of contour extraction are shown in Figure 2.5. These examples show that due to the stopping term, the noise present in the fingerprint image has very little effect on contour extraction.

Once the contour extraction algorithm provides final fingerprint contour $\bar{\psi}$, it is scanned using the standard contour tracing technique [70] from top to bottom and left to right consecutively to classify the fingerprint features as: pores, ridges, and dots. The tracing technique uses the following model-based approach:

1. A blob of size greater than 2 pixels and less than 40 pixels is classified as a pore. Therefore, noisy contours, which are sometimes wrongly extracted, are not included in the feature set. A pore is approximated with a circle and the center is used as the pore feature. 

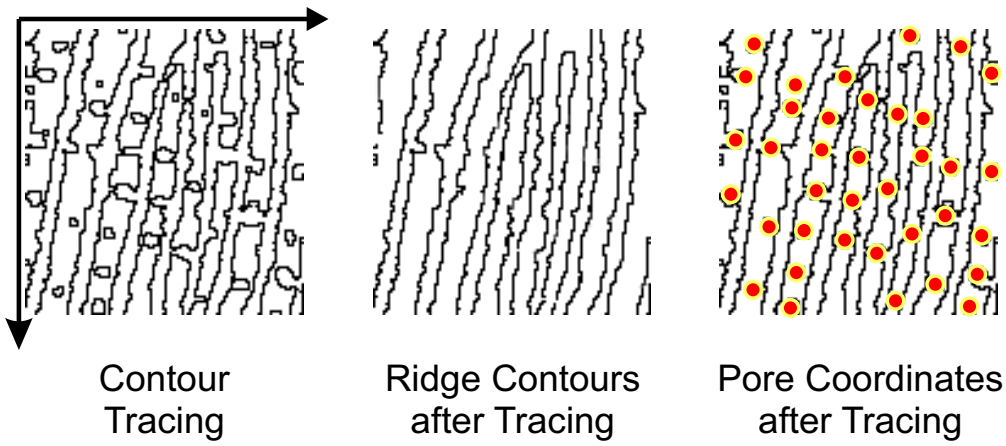

Figure 2.6: Tracing of fingerprint contour and categorization of level-3 pore and ridge features.

2. The ridge contour (edge of a ridge) features are the $x, y$ coordinates of the pixel and direction of the contour at that pixel.

3. Any blob of size less than 0.02 " that does not lie on a ridge is marked as a dot and the corresponding $x, y$ coordinates are stored.

4. If a blob or a ridge structure whose width is substantially thinner (less than $40 \%$ of average local ridge width) and size is greater than $0.02 "$, then it is marked as an incipient ridge. Further, if the incipient ridge is a series of clearly separated dots, then these are marked as separate incipient ridges. The $x, y$ coordinate of the endpoints and the distance are stored as the incipient ridge features.

These features and tracing procedure follow the standards defined by the ANSI/NIST CDEFFS [113]. As shown in Figure 2.6, fine details and structures present in the fingerprint contour are traced and categorized into level-3 features depending on the shape and attributes. Additional examples of tracing algorithms are shown in Figures 2.4(d), $2.7,2.8$ and 2.9 .

Each of the level-3 features are separately stored in a matrix. The size of each feature matrix is dependent on the size of the fingerprint image and features present in 

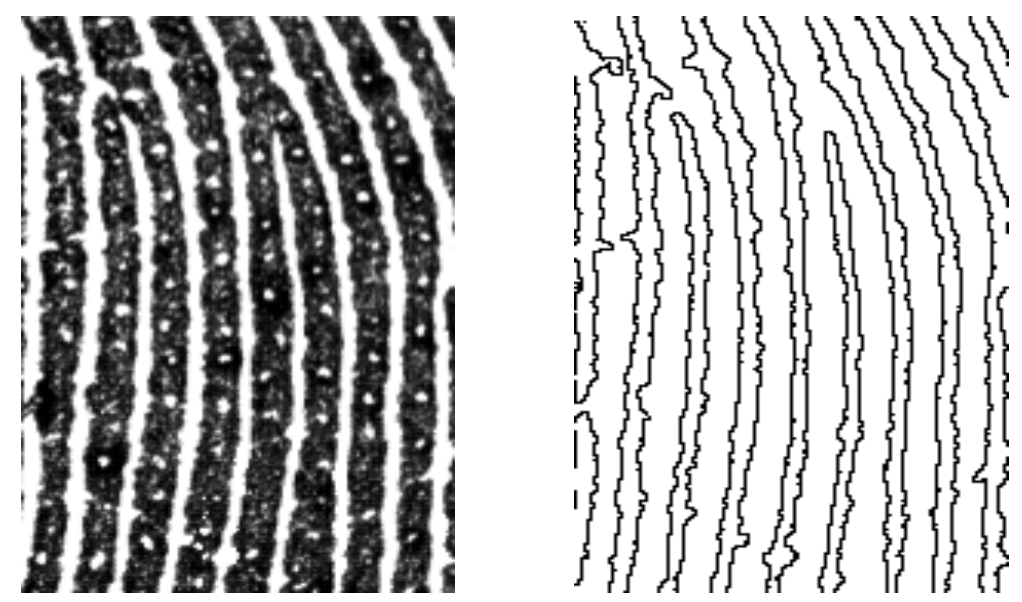

Figure 2.7: Ridge contours extracted using the proposed algorithm.
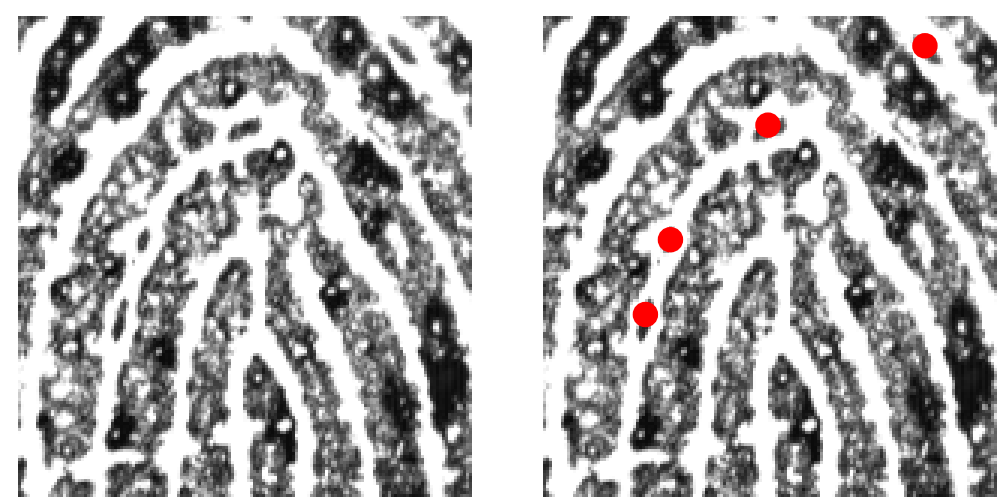

Figure 2.8: $x, y$ coordinates of the dots (red) are stored as dot features.
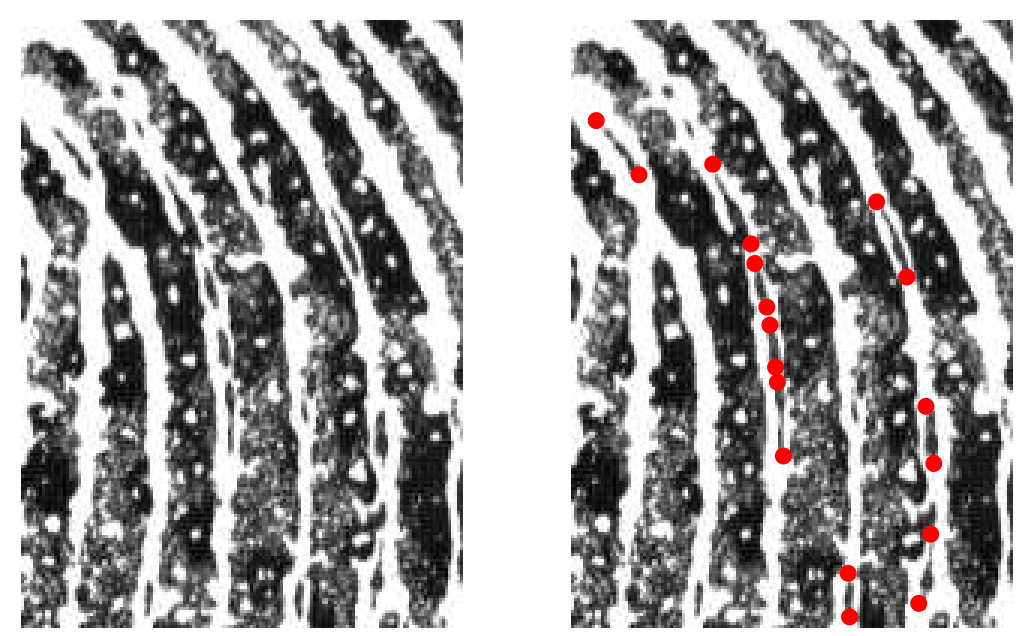

Figure 2.9: End points of incipient ridges (red) are marked after tracing the contour. 
it. For matching, Mahalanobis distance between each level-3 feature matrices pertaining to the gallery and probe images is computed. Mahalanobis distance between two vectors $\mathbf{f}_{\mathbf{G}}$ and $\mathbf{f}_{\mathbf{P}}$ is defined as,

$$
M D\left(\overline{\mathbf{f}_{\mathrm{G}}}, \overline{\mathbf{f}_{\mathbf{P}}}\right)=\sqrt{\left(\overline{\mathbf{f}_{\mathrm{G}}^{-}}-\overline{\mathbf{f}_{\mathbf{P}}}\right)^{t} S^{-1}\left(\overline{\mathbf{f}_{\mathrm{G}}}-\overline{\mathbf{f}_{\mathbf{P}}}\right)}
$$

where, $\overline{\mathbf{f}_{\mathbf{G}}}$ and $\overline{\mathbf{f}_{\mathbf{P}}}$ are the two features vectors to be matched, and $S$ is the positive definite covariance matrix of $\overline{\mathbf{f}_{\mathbf{G}}}$ and $\overline{\mathbf{f}_{\mathbf{P}}}$. Mahalanobis distance $M D\left(\overline{\mathbf{f}_{\mathbf{G}}}, \overline{\mathbf{f}_{\mathbf{P}}}\right)$ is used as the match score of level-3 features. It ensures that the features having high variance do not contribute to the distance and hence decrease the false reject rate for a fixed false accept rate. Using this measure, four Mahalanobis distance scores, $S D(i)(i=1, \cdots, 4)$, associated with the individual level-3 features are computed.

To compute a level-3 feature based match score, we next propose the use of qualitybased likelihood ratio [67] approach. Quality score vector, q, for the fingerprint image and distance score, $S D(i)$, are given as input to the Gaussian Mixture Model based density estimation algorithm [119]. For a gallery-probe pair, let $g_{g e n}(S D(i), \mathbf{q})$ and $g_{i m p}(S D(i), \mathbf{q})$ be the genuine and impostor joint marginal densities respectively. The quality-based likelihood ratio for a gallery-probe pair is computed using Equation 2.10,

$$
S=\sum_{i=1}^{4} \frac{g_{g e n}(S D(i), \mathbf{q})}{g_{i m p}(S D(i), \mathbf{q})}
$$

Finally, the decision of accept or reject is made using the threshold $t$.

$$
\text { Decision }= \begin{cases}\text { accept } & \text { if } \quad S \geq t \\ \text { reject } & \text { otherwise }\end{cases}
$$




\subsection{Database and Algorithms used for Evaluation}

The performance of the proposed level-3 feature extraction and matching algorithm is evaluated and compared with existing level-3 feature based fingerprint recognition algorithms on 1000 ppi fingerprint databases. In this section, we briefly describe the database and the algorithms used for validation.

\subsubsection{Fingerprint Database}

To evaluate the proposed quality induced level-3 feature extraction and matching algorithm, two sets of fingerprint databases are used. The first fingerprint database, obtained from law enforcement agencies, contains images from 550 different classes. For each class, there are five rolled and five slap fingerprints. The resolution of fingerprint images is 1000 ppi to facilitate the extraction of both level- 2 and level-3 features. The second fingerprint database is a non-ideal database prepared by the authors that contains 1000 ppi images pertaining to 150 classes and for each class there are 10 slap fingerprint images. Further, from each class of the first database, one good and one bad quality rolled fingerprints, and one good and one bad quality slap fingerprints are selected for training and the remaining images are used for testing. Therefore, we have 1100 rolled and 1100 slap images for training and 1650 rolled and 1650 slap images for testing. Similarly, from the second database, 300 good quality and 300 bad quality slap images $(150 \times 4)$ are selected for training and rest of the 900 images $(150 \times 6)$ are used as test data. Using the testing database, we perform three sets of experiments,

1. Matching rolled fingerprints with rolled fingerprints from 550 classes with three images for each class.

2. Matching rolled fingerprints with slap fingerprints from 550 classes with three im- 
ages for each class.

3. Matching slap fingerprints with slap fingerprints from 150 classes with six images for each class.

\subsubsection{Existing Algorithms}

To compare the performance of the proposed level-3 algorithm with existing algorithms, we have implemented two algorithms. The algorithm by Kryszczuk et al. [58, 59] extracts pore information from high resolution fingerprint images by applying different techniques such as correlation based alignment, Gabor filtering, binarization, morphological filtering, and tracing. The match score obtained from this algorithm is a normalized similarity score in the range of $[0,1]$. The algorithm proposed by Jain et al. [45] uses Gabor filtering and wavelet transform for pore and ridge feature extraction and iterative closest point for matching.

\subsection{Experimental Evaluation}

The performance of the proposed algorithms is evaluated using the Receiver Operating Characteristics curves that are generated by computing the genuine accept rates at different false accept rates. Training datasets are used to learn the Gaussian Mixture Model for density estimation algorithm. As mentioned in Section 2.2.1, three sets of experiments are performed using the testing datasets.

We compute the verification performance of the proposed level-3 feature extraction algorithm and compare it with existing level-2 [48, 55] and level-3 feature based verification algorithms $[45,58,59]$. The ROC plots in Figures $2.10-2.12$ and verification

accuracies in Table 2.1 summarize the results of the three experiments: matching rolled 
fingerprint, matching rolled images with slap images, and matching slap fingerprints. The key results and analysis of our experiments are summarized below.

1. For matching rolled fingerprints (Experiment 1), the proposed level-3 feature extraction algorithm yields a verification accuracy of $93.9 \%$ which is around $2-7 \%$ better than existing algorithms. Existing level-2 minutiae based verification algorithm is more optimized for 500 ppi fingerprint images and the performance does not improve when 1000 ppi images are used. On the other hand, the level-3 pore matching algorithm $[58,59]$ requires very high quality fingerprint images $(\geq 2000$ ppi) for feature extraction and matching, and the performance suffers when the fingerprint quality is poor or the amount of pressure applied during scanning affects the pore information. The algorithm proposed by Jain et al. [45] is efficient with good quality rolled fingerprint images. However, the verification accuracy decreases with poor quality fingerprints when pore features are not clearly visible. The proposed level set based feature extraction algorithm is robust to irregularities due to noise and efficiently compensates the variations in pressure during fingerprint capture by incorporating ridge, pore, and dot features. Mahalanobis distance measure also reduces the false reject cases thus improving the verification accuracy.

2. The experiments with rolled to slap fingerprint matching (Experiment 2) show a decrease of around $1.1-6.3 \%$ in the verification accuracy of the proposed and existing algorithms. The proposed approach exhibits best verification performance of $92.4 \%$. The experimental results show that the proposed level-3 feature extraction algorithm provides good recognition performance even with limited number of minutiae.

3. Experiments with slap fingerprints (Experiment 3) show the main advantage of 
the proposed algorithm. Since the database contains several non-ideal poor quality fingerprint images, verification accuracy of existing level-3 feature based algorithms reduce significantly. The decrease in verification performance is mainly because of the poor quality images which cause either missing pore features or spurious pores. The proposed algorithm, conversely, manages to yield $85.2 \%$ accuracy which is at least $5 \%$ better than existing algorithms. The improved performance is because the feature extraction algorithm, most of the times, provides correct feature boundary, and the quality factor and likelihood ratio for decision making further improve the verification accuracy. Moreover, the quality-based likelihood ratio achieves optimal performance by satisfying the Neyman-Pearson theorem [31].

4. The experiments also show that, in general, level-2 and level-3 algorithms provide correct results when both the gallery and probe are rolled fingerprint images. Similarly, when the images are good quality slap, both level-2 and level-3 features yield correct results (Figure 2.13(a)). However, there are several slap fingerprints in the database that are very difficult to recognize using level-2 features because of the inherent fingerprint structure. One such example is shown in Figure 2.13(b) where level-2 feature extraction algorithm does not yield correct result but the proposed level-3 feature extraction algorithm provides correct results. Further, there are cases in which both level-2 and level-3 algorithms fail to perform (Figure 2.13(c)) because of sensor noise and less pressure. Such cases require image quality enhancement algorithms to enhance the quality such that feature extraction algorithm can extract useful features for verification.

5. During the experiments, we also analyzed the verification performance of individual level-3 features. It is observed that among the level-3 features, dots and incipient 


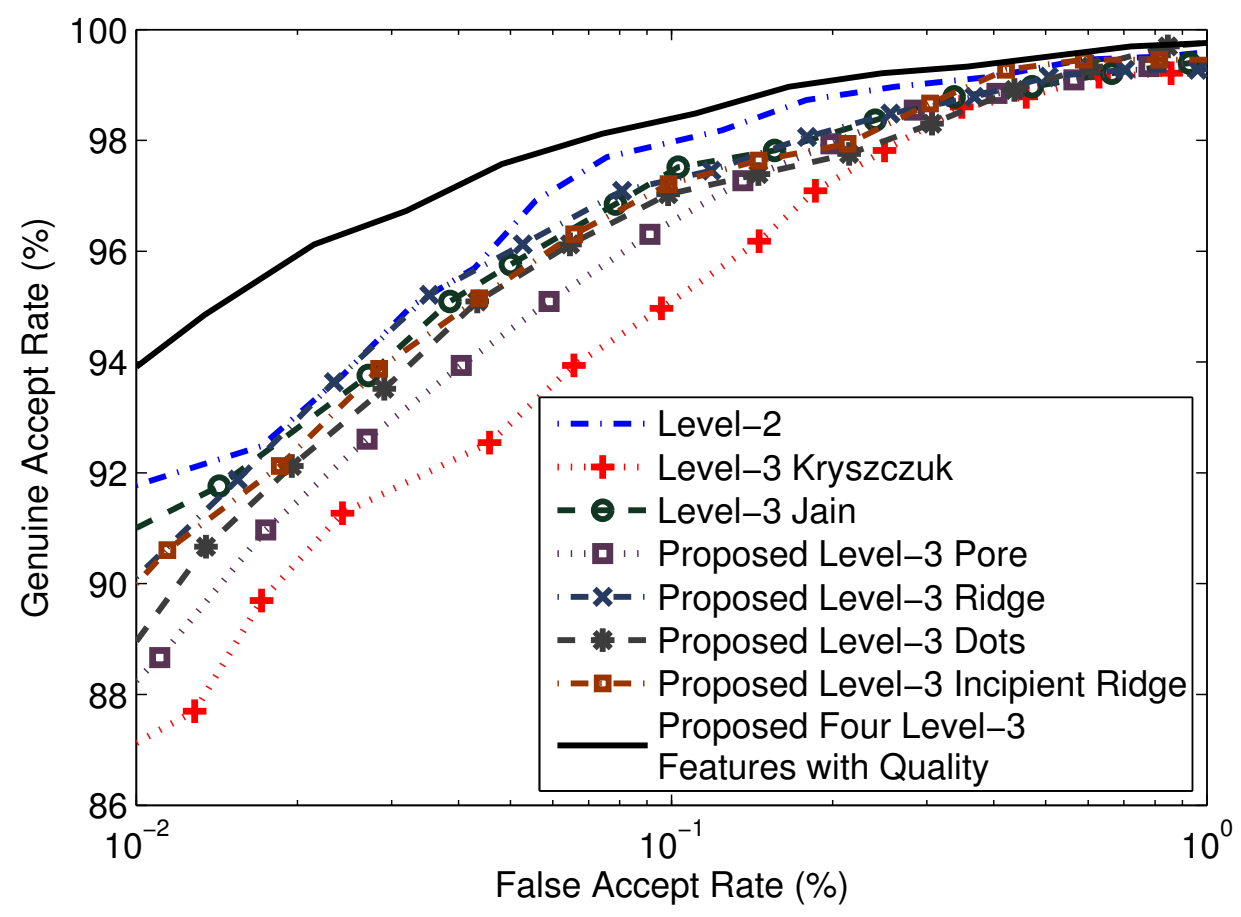

Figure 2.10: Matching rolled fingerprints: ROC plot for the proposed level-3 feature extraction and comparison with existing level-3 feature based algorithms $[45,58,59]$ and minutiae based algorithm.

ridges are the most stable and pores (specifically pore shape and size) are the least stable features.

6. Computationally, the proposed curve evolution based level-3 feature extraction algorithm is fast and requires around 3 seconds for contour extraction and tracing on a $2 \mathrm{GHz}$ Pentium Duo Core processor with $2 \mathrm{~GB}$ RAM under MATLAB environment. The quality induced fingerprint recognition algorithm requires 5 seconds for verification which is around 2 seconds slower that the level-2 feature based algorithm. However, the improvement in accuracy is notably high with a small computational overhead. 


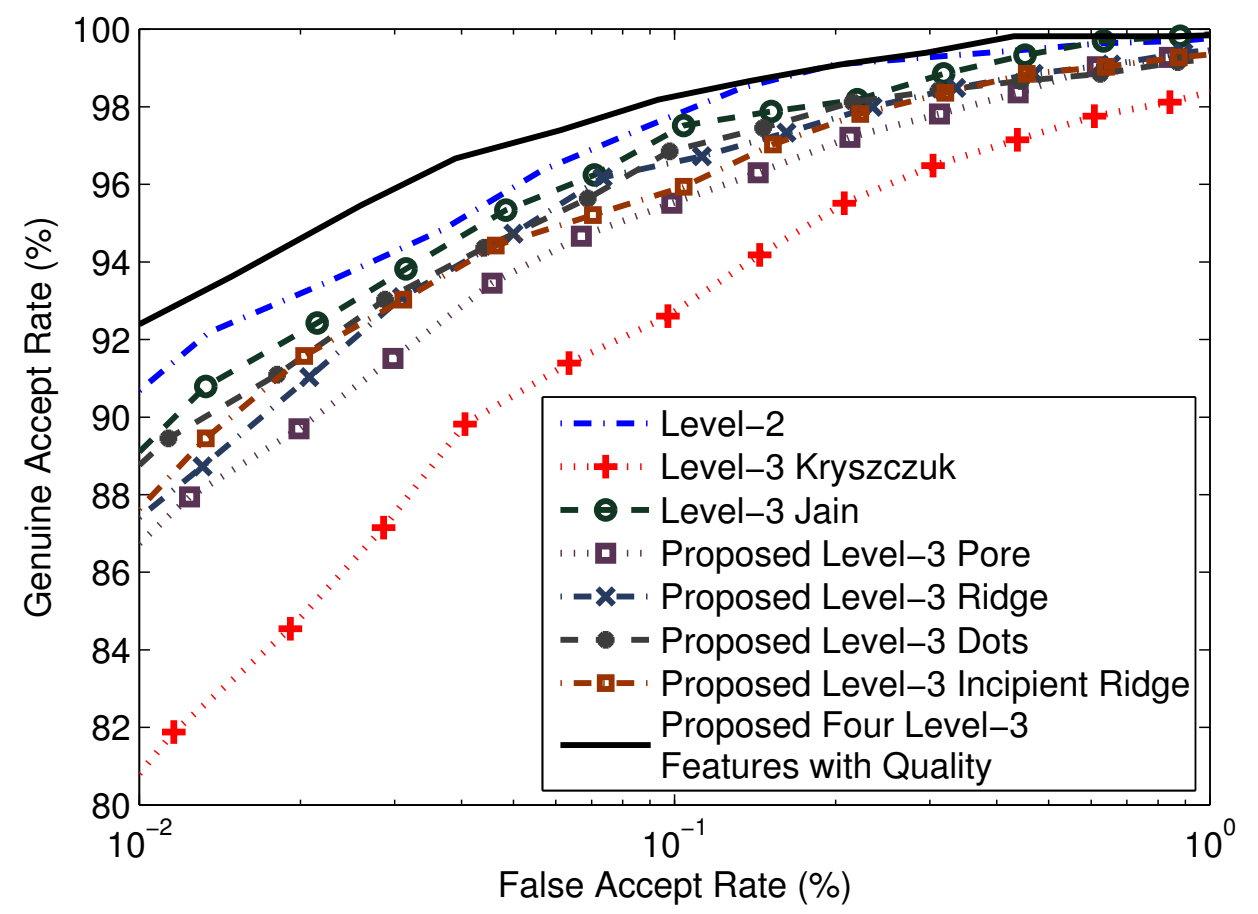

Figure 2.11: Matching rolled to slap fingerprints: ROC plot for the proposed level-3 feature extraction and comparison with existing level-3 feature based algorithms [45, 58, 59] and minutiae based algorithm. 


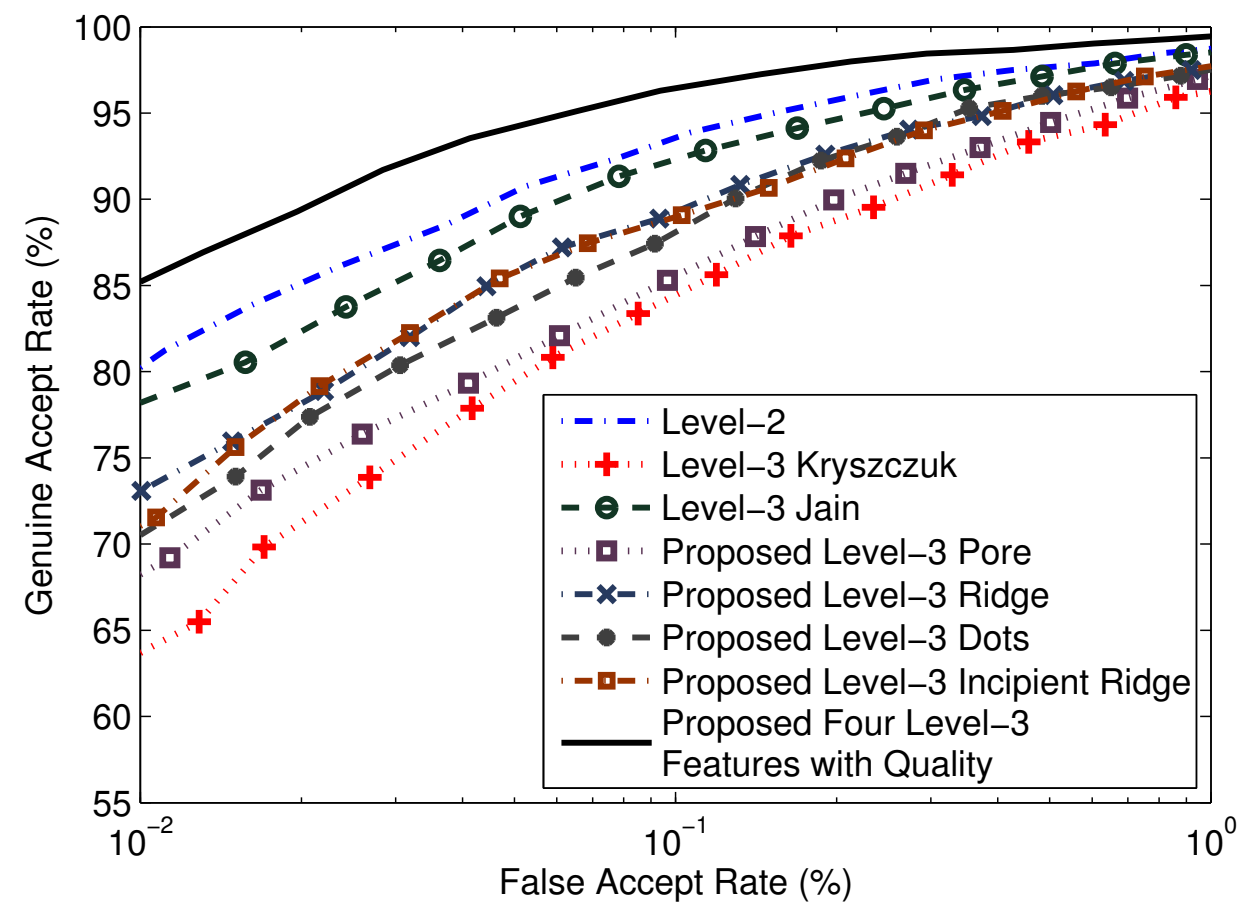

Figure 2.12: Matching slap fingerprints: ROC plot for the proposed level-3 feature extraction and comparison with existing level-3 feature based algorithms $[45,58,59]$ and minutiae based algorithm.

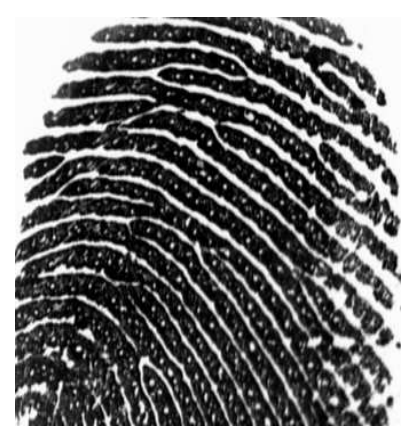

(a)

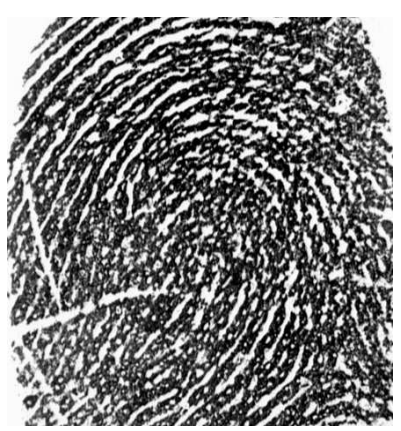

(b)

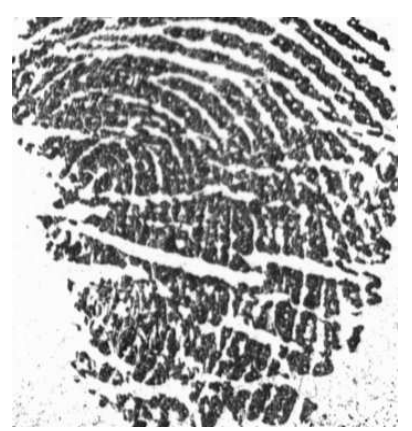

(c)

Figure 2.13: Three sample cases: (a) when both level-2 and level-3 features based algorithms provide correct result, (b) when level-2 feature based algorithm fails to provide correct result whereas level-3 features based algorithm provides accurate decision, and (c) when both the algorithms fail to perform. 
Table 2.1: Comparing verification performance of the proposed quality induced level-3 feature extraction and matching algorithm with existing feature extraction algorithms using fingerprints with varying number of features. Verification accuracy is computed with $\mathrm{FAR}=0.01 \%$.

\begin{tabular}{|l|c|c|c|c|}
\hline Algorithm & $\begin{array}{c}\text { Rolled } \\
\text { to Rolled } \\
\text { Fingerprint }\end{array}$ & $\begin{array}{c}\text { Rolled } \\
\text { to Slap } \\
\text { Fingerprint }\end{array}$ & $\begin{array}{c}\text { Slap } \\
\text { to Slap } \\
\text { Fingerprint }\end{array}$ & $\begin{array}{c}\text { Average } \\
\text { Time } \\
\text { seconds) }\end{array}$ \\
\hline Level-2 Minutiae [48], [55] & $91.8 \%$ & $90.7 \%$ & $80.2 \%$ & 03 \\
\hline Level-3 Pores [58], [59] & $87.2 \%$ & $80.9 \%$ & $63.7 \%$ & 12 \\
\hline Level-3 Pore and Ridge [45] & $91.0 \%$ & $89.1 \%$ & $78.1 \%$ & 34 \\
\hline Proposed Level-3 Pore & $88.3 \%$ & $86.8 \%$ & $68.3 \%$ & 03 \\
\hline Proposed Level-3 Ridge & $91.2 \%$ & $87.4 \%$ & $73.0 \%$ & 03 \\
\hline Proposed Level-3 Dots & $88.9 \%$ & $88.7 \%$ & $70.5 \%$ & 01 \\
\hline Proposed Level-3 Incipient Ridge & $90.0 \%$ & $87.6 \%$ & $71.1 \%$ & 01 \\
\hline $\begin{array}{l}\text { Proposed Quality } \\
\text { Induced Level-3 Features }\end{array}$ & $93.9 \%$ & $92.4 \%$ & $85.2 \%$ & 05 \\
\hline
\end{tabular}




\subsection{Summary}

With the availability of high resolution fingerprint sensors, salient features such as pores, ridge contours, and dots are prominently visible. In this research, these conspicuous level-3 features are utilized for fingerprint verification. RDWT based quality assessment algorithm is used to compute the image quality score followed by a two stage registration algorithm to register the gallery and probe fingerprint images. A fast feature extraction algorithm is proposed to extract detailed level-3 pore, ridge, and dot features using level set curve evolution approach. Experimental results on a high resolution fingerprint database demonstrate the effectiveness of the proposed algorithm with respect to both accuracy and time. The experiments also show that the proposed algorithm outperforms existing level-2 and level-3 feature based fingerprint recognition algorithms. 


\section{Chapter 3}

\section{Integrating Image Quality in Level-2 and Level-3 Fingerprint Match Score}

\section{Fusion}

The paradigm of information fusion, that entails the consolidation of evidence presented by multiple sources, has been successfully used to enhance the recognition performance of biometric systems. The use of multiple pieces of evidences in order to deduce or verify human identity is often referred to as multibiometrics. While fusion can be accomplished at several different levels in a biometric system [47], fusion at the match score level has been extensively studied in the literature primarily due to (a) the ease of accessing match scores using commercial software, and (b) the trade-off that it offers in terms of information complexity compared to data level fusion (too much information) and decision level fusion (too little information). Fusion at the match score level involves combining the match scores generated by multiple classifiers (or matchers) to render a decision about the identity of the subject.

There are several match score fusion algorithms in literature that fuse two or more 
biometric information such as match scores obtained from level-2 and level-3 features. These algorithms are generally based on statistical rules such as sum rule [56, 76], minmax rule [56, 76], product rule [56, 76], learning techniques such as SVM fusion [1], or belief model techniques such as Dempster-Shafer theory fusion [5, 85]. While existing match score fusion algorithms have been demonstrated to be effective in several applications, their matching performance is compromised when participating biometric classifiers generate conflicting results.

In context to the research on level-2 and level-3 features based recognition, we observe that the match scores obtained from these two matching algorithms have limited correlation (Figure 3.1). This analysis suggests that recognition accuracy can be further improved if these match scores are fused. In this chapter, we propose five match score fusion algorithms that use quality score along with match scores for efficiently fusing level-2 and level-3 match scores. The first algorithm combines level-2 and level-3 features using Delaunay triangulation technique to compute a feature supervector containing nine topological measures. The algorithm also incorporates quality based likelihood ratio for efficient classification and fusion. The algorithm is able to tolerate minor deformation of features and non-linearity in the fingerprint information. The second algorithm is evidence theoretic sum rule which estimates the genuine and impostor density using basic probability assignments computed from the match scores and incorporates prior evidences such as image quality and verification accuracy computed using the training dataset. The third algorithm utilizes $2 \nu$-Support Vector Machine to learn the inter-class and intra-class variations in the match scores and fuse them for classification. The fourth algorithm is based on belief models and utilizes the Proportional Conflict Redistribution rule $[27,89]$ to model the uncertainties in the matcher. The fifth fusion algorithm is the sequential fusion algorithm that combines three paradigms of match score fusion 


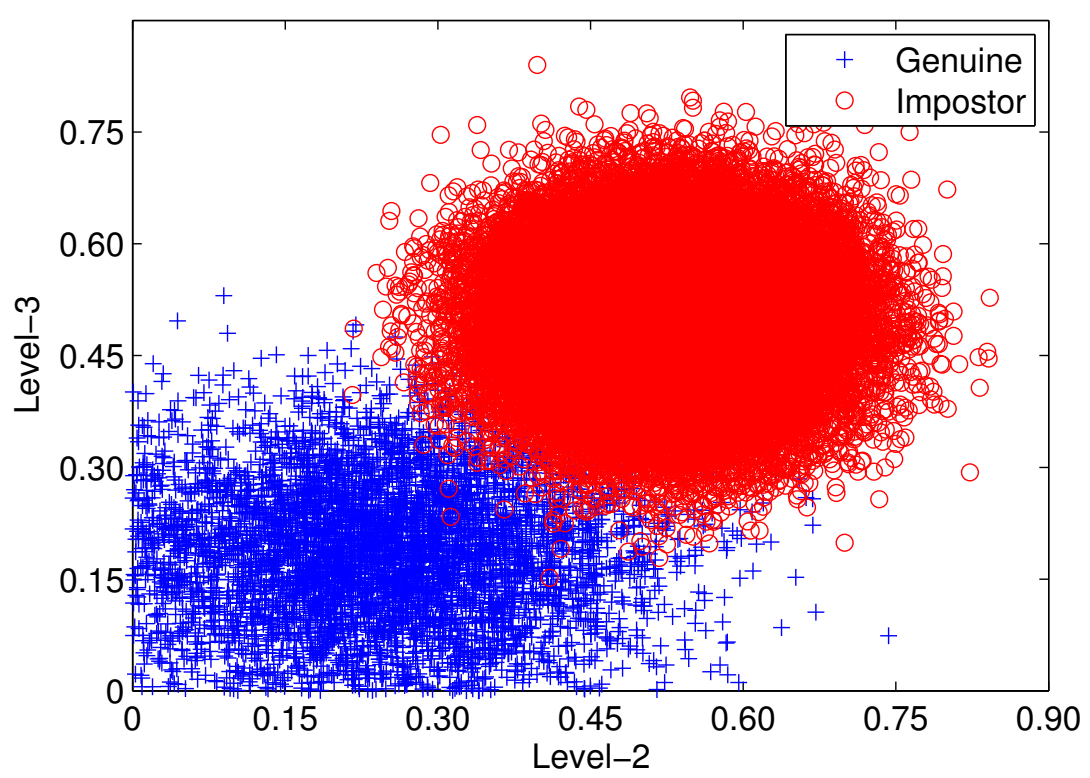

Figure 3.1: Scatter plot of match scores corresponding to the level-2 and level-3 fingerprint features.

algorithms: statistical, learning, and belief model. Subsequent sections describe these fusion algorithms in detail.

\subsection{Fusion of Level-2 and Level-3 Features using De- launay Triangulation and Quality Incorporated Likelihood Ratio}

In this section, we describe the proposed Delaunay triangulation based fusion algorithm for combining level-2 and level-3 information such that it is resilient to minor deformations in features and missing information. Fingerprint features, both level-2 and level-3, are extracted using the feature extraction algorithms described in the previous 


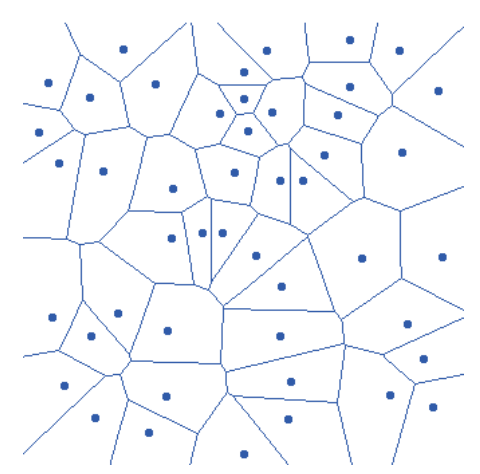

(a)

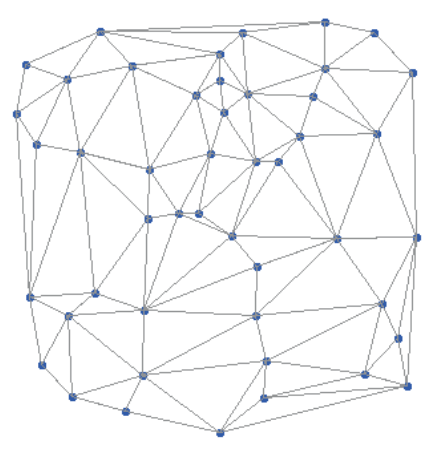

(b)

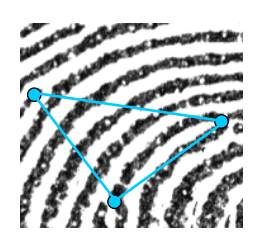

(c)

Figure 3.2: Example of (a) Voronoi diagram of fingerprint minutiae, (b) Delaunay triangulation of fingerprint minutiae, (c) minutiae triplet.

chapter. The proposed fusion algorithm starts with computing Delaunay triangulation using level-2 minutiae. Delaunay triangulation has been used with level-2 features for fingerprint indexing and identification $[11,77,103]$. However, in the proposed fingerprint fusion algorithm, Delaunay triangulation is used to generate a feature supervector that contains both level-2 and level-3 features. A Delaunay triangle is formed using minutiae information as follows:

1. Given $n$ minutiae points, the Voronoi diagram is computed which decomposes the minutiae points into different regions.

2. Voronoi diagram is used to compute the Delaunay triangulation by joining the minutiae coordinates present in the neighborhood Voronoi regions. Figures 3.2(a) and (b) show an example of Voronoi diagram and Delaunay triangulation of fingerprint minutiae.

Each triangle in the Delaunay triangulation is used as a minutiae triplet [14]. Figure 3.2(c) shows an example of a minutiae triplet along with level-3 features. Tuceryan and Chorzempa [103] found that Delaunay triangulation has the best structural stability and 
hence minutiae triplets computed from Delaunay triangulation are able to sustain the variations due to fingerprint deformation [11]. Further, Bebis et al. [11] and Ross and Mukherjee [77] have shown that any local variation due to noise or insertion/deletion of feature points affects the Delaunay triangulation only locally.

From the minutiae triplets generated using the Delaunay triangulation, the feature supervector that includes minutia, pore, and ridge information is computed. Our previous experiments and results from Jain et al. [45] show that pore features are less reliable compared to minutiae and ridge features. Based on these studies, the feature supervector in the proposed fusion algorithm is composed of nine elements which are described as follows:

1. Average cosine angle in minutiae triplet $(A)$ : Let $\alpha_{\min }$ and $\alpha_{\max }$ be the minimum and maximum angles in a minutiae triplet. Average cosine angle of the minutiae triplet is defined as,

$$
A=\cos \left(\alpha_{\text {avg }}\right)
$$

where $\alpha_{\text {avg }}=\left[\alpha_{\min }+\alpha_{\max }\right] / 3$. Average cosine angle vector is computed for all the minutiae triplets in a given fingerprint.

2. Triangle orientation $(O)$ : According to Bhanu and Tan [14], triangle orientation is defined as $\phi=\operatorname{sign}\left(z_{21} \times z_{32}\right)$, where $z_{21}=z_{2}-z_{1}, z_{32}=z_{3}-z_{2}, z_{13}=z_{1}-z_{3}$, and $z_{i}=x_{i}-j y_{i} . z_{i}$ is computed from the coordinates $\left(x_{i}, y_{i}\right), i=1,2,3$ in the minutiae triplet. Triangle orientation vector, $O$, is then computed for all the minutiae triplets in a given fingerprint.

3. Triplet density $(T D)$ : If there are $n^{\prime}$ minutiae in a Voronoi region centered at a minutia, then the minutiae density is $n^{\prime}$. For a minutiae triplet, we define triplet 
density as the average minutiae density of the three minutiae that form the triplet. If $n_{1}^{\prime}, n_{2}^{\prime}$, and $n_{3}^{\prime}$ are the minutiae density of the three minutiae, then the triplet density is $\left(n_{1}^{\prime}+n_{2}^{\prime}+n_{3}^{\prime}\right) / 3$. We compute triplet density for all the triplets in the fingerprint image to form the triplet density vector $T D$.

\section{Min-Max distance between minutia points and $k$-nearest neighbor pores} $\left(M_{p}\right)$ : For every minutia point, we first compute the distances between minutiae and its $k$-nearest neighboring pores which are on the same ridge. Out of these $k$ distances, the minimum and maximum distances are used as the fusion parameters. Min-Max distance vector $\left(M_{p}\right)$ is then generated from all the minutiae in a fingerprint image.

5. Average distance of $k$-nearest neighbor pores $\left(P_{a v g}\right)$ : Average distance vector of $k$-nearest neighbor pores, $P_{a v g}$, is formed by computing the average distance of $k$-nearest neighbor pores of all the minutiae in a triplet.

6. Average ridge width $\left(R_{w}\right)$ : Ridge width is computed for each minutiae triplet using the tracing technique [70]. Average ridge width vector $\left(R_{w}\right)$ is computed by taking the average of the ridge width of each minutiae triplet.

7. Ridge curve parameters $\left(R_{C}\right)$ : Ross and Mukherjee [77] proposed the use of ridge curve parameters for fingerprint identification. We modified the ridge curve parameter for the proposed fusion algorithm. Each ridge can be parameterized as $y=a x^{2}+b x+c$ where $a, b$, and $c$ are the parameterized coefficients. Ridge

curve parameter for a ridge is $\left[\frac{a}{a+b+c}, \frac{b}{a+b+c}, \frac{c}{a+b+c}\right]$. In a minutiae triplet, each minutiae is associated with a ridge. Therefore the ridge curve parameters of a minutiae triplet are $\left[\frac{a_{i}}{a_{i}+b_{i}+c_{i}}, \frac{b_{i}}{a_{i}+b_{i}+c_{i}}, \frac{c_{i}}{a_{i}+b_{i}+c_{i}}\right]$ where $i=1,2$, and 3 . Ridge curve parameter $R_{C}$ is similarly computed for all the minutiae triplets. 
8. Dot $(D)$ : If dots are present in the fingerprint image, then the position of each dot is stored in the vector $D$.

9. Incipient Ridges $I$ : If incipient ridges are present in the fingerprint image, then the incipient ridge features are stored in the vector $I$.

A feature supervector $F\left(\mathbf{A}, \mathbf{O}, \mathbf{T D}, \mathbf{M}_{\mathbf{p}}, \mathbf{P}_{\mathbf{a v g}}, \mathbf{R}_{\mathbf{w}}, \mathbf{R}_{\mathbf{C}}, \mathbf{D}, \mathbf{I}\right)$ is formed by concatenating these nine elements (vectors) that include information pertaining to both level-2 and level-3 fingerprint features. For matching this supervector, Mahalanobis distance associated with the individual parameters are computed. Let $M D(i)$ be the Mahalanobis distance and $i=1, \ldots, 9$ (for parameters $\mathbf{A}, \mathbf{O}, \mathbf{T D}, \mathbf{M}_{\mathbf{p}}, \mathbf{P}_{\mathbf{a v g}}, \mathbf{R}_{\mathbf{w}}, \mathbf{R}_{\mathbf{C}}, \mathbf{D}$, and $\mathbf{I}$ ). The fused match score, $S_{f u s e d}$, is computed by applying Sum rule to the vector MD, i.e,

$$
S_{\text {fused }}=\sum_{i=1}^{9} M D(i)
$$

We next propose incorporating quality-based likelihood ratio in the match scores for classification. Quality score vector, $\mathbf{q}$, for the probe image and the match score, $S_{\text {fused }}$, are given as input to the Gaussian Mixture Model based density estimation algorithm [119]. For a gallery-probe pair, let $g_{\text {gen }}\left(S_{\text {fused }}, \mathbf{q}\right)$ and $g_{\text {imp }}\left(S_{\text {fused }}, \mathbf{q}\right)$ be the genuine and impostor joint marginal densities respectively. The quality-based likelihood ratio for a gallery-probe pair is computed using Equation 3.3 and a threshold is applied on $S_{\text {final }}$ for genuine or impostor classification.

$$
S_{\text {final }}=\frac{g_{\text {gen }}\left(S_{\text {fused }}, \mathbf{q}\right)}{g_{\text {imp }}\left(S_{\text {fused }}, \mathbf{q}\right)}
$$




\subsection{Evidence Theoretic Sum Rule Fusion}

Delaunay triangulation based fusion algorithm is effective but it is also computationally complex. Further, the algorithm relies on correct level-2 feature extraction to form Delaunay triangle. Spurious or missing minutiae can affect computing feature supervector. In biometrics literature, sum rule has been used as a simple yet effective fusion scheme. Moreover, it has been suggested that incorporating image quality score in sum rule improves the verification performance. Therefore, in this section, we present an evidence theoretic sum rule algorithm for combining image quality and match scores obtained from level-2 and level-3 features. First we describe the formulation of the evidence-theoretic sum rule using belief functions followed by the fusion algorithm. Since biometric verification is a two-class problem with the classes being genuine and impostor, the fusion algorithm is formulated as a two-class problem. Belief functions are defined on the frame of discernment that consists of a finite set of exhaustive and mutually exclusive hypothesis. Let $\Theta=\left\{\theta_{\text {gen }}, \theta_{i m p}\right\}$ be the frame of discernment and $\theta_{\text {gen }}$ and $\theta_{\text {imp }}$ be the hypothesis belonging to genuine and impostor classes respectively.

\subsubsection{Formulation of Evidence Theoretic Sum Rule}

In the evidence theoretic sum rule, the belief function which is also known as the basic probability assignment $(b p a)$ is defined as $\bar{m}(\cdot): \Theta \rightarrow[0,1]$, such that $\bar{m}\left(\theta_{g e n}\right)+$ $\bar{m}\left(\theta_{i m p}\right)=1$. Here, $\bar{m}\left(\theta_{\text {gen }}\right)$ represents the belief of data being genuine and $\bar{m}\left(\theta_{\text {imp }}\right)$

represents the belief of data being impostor. For a given match score, if $\bar{m}\left(\theta_{\text {gen }}\right)=0.7$ then $\bar{m}\left(\theta_{\text {imp }}\right)=0.3$.

Figure 3.3 shows the steps involved in the proposed evidence theoretic sum rule. To fuse match scores obtained from two different classifiers, the match scores are first 


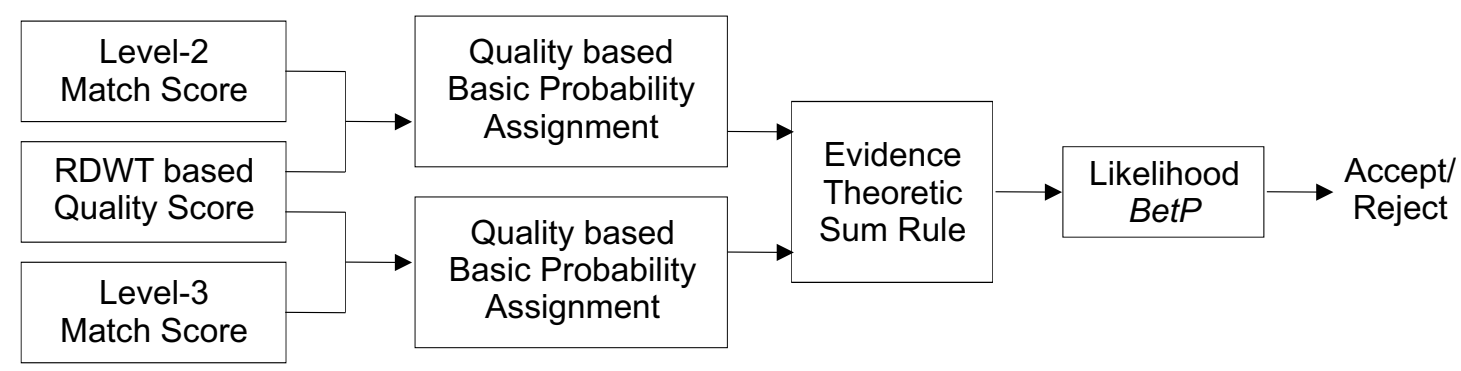

Figure 3.3: Illustrating the steps involved in the proposed evidence theoretic sum rule.

transformed into basic probability assignments, $\bar{m}_{1}(\cdot)$ and $\bar{m}_{2}(\cdot)$, and then fused using Equations 3.4 and 3.5.

$$
\begin{aligned}
\bar{m}_{\text {fused }}\left(\theta_{\text {gen }}\right) & =\frac{\bar{m}_{1}\left(\theta_{\text {gen }}\right)+\bar{m}_{2}\left(\theta_{\text {gen }}\right)}{2} \\
\bar{m}_{\text {fused }}\left(\theta_{\text {imp }}\right) & =\frac{\bar{m}_{1}\left(\theta_{\text {imp }}\right)+\bar{m}_{2}\left(\theta_{\text {imp }}\right)}{2}
\end{aligned}
$$

A decision to accept or reject is made using the pignistic probability $(B e t P)$ and likelihood ratio test.

$$
\operatorname{BetP}\left(\theta_{i}\right)=\sum_{\theta_{i} \in A \subseteq \Theta} \frac{m_{\text {fused }}(A)}{|A|}
$$

where $i=\{g e n, i m p\}$. Pignistic probability transforms belief assignment into probability assignments and likelihood ratio test is used with the threshold $t$ for decision making as shown in Equation 3.7.

$$
\text { Decision }= \begin{cases}\text { genuine } & \text { if } \quad \frac{\operatorname{Bet} P\left(\theta_{\text {gen }}\right)}{\operatorname{BetP}\left(\theta_{i m p}\right)} \geq t \\ \text { impostor } & \text { otherwise }\end{cases}
$$




\subsubsection{Match Score Fusion using Evidence-Theoretic Sum Rule}

Let $s_{1}$ and $s_{2}$ be the two match scores to be fused. We found that the distribution of match scores for every element of $\Theta=\left\{\theta_{\text {gen }}, \theta_{i m p}\right\}$ follows a Gaussian distribution as shown in Equation 3.8.

$$
p\left(s_{i}, \bar{\mu}_{i j}, \bar{\sigma}_{i j}\right)=\frac{1}{\bar{\sigma}_{i j} \sqrt{2 \pi}} \exp \left[-\frac{1}{2}\left\{\frac{s_{i}-\bar{\mu}_{i j}}{\bar{\sigma}_{i j}}\right\}^{2}\right]
$$

where $\bar{\mu}_{i j}$ and $\bar{\sigma}_{i j}$ are the mean and standard deviation of the $i^{\text {th }}$ classifier corresponding to the $j^{\text {th }}$ element of $\Theta$. Therefore the Gaussian distribution is used to compute the basic probability assignment $\bar{m}_{i}(j)$,

$$
\bar{m}_{i}(j)=\frac{p\left(s_{i}, \bar{\mu}_{i j}, \bar{\sigma}_{i j}\right) \bar{\beta}_{i j}}{\sum_{j=1}^{\Theta} p\left(s_{i}, \bar{\mu}_{i j}, \bar{\sigma}_{i j}\right) \bar{\beta}_{i j}}
$$

where $\bar{\beta}_{i j}$ is the weight factor of classifier $i$ corresponding to the $j^{t h}$ element of $\Theta$. $\bar{\beta}_{i j}$ is defined as,

$$
\bar{\beta}_{i j}=q_{m e d} \bar{V}_{i j}
$$

Here, $q_{m e d}$ is the median of quality score vector of the input probe image and $\bar{V}_{i j}$ is the verification accuracy prior computed on the training database. Verification accuracy prior is used to attune the classifier's reliability of a probe match by means of errors made on the representative training dataset. Quality score vector is computed using the redundant discrete wavelet transform based quality assessment algorithm described in Chapter 2 and median of the quality score vector is used in the fusion algorithm. Values of both $q_{m e d}$ and $\bar{V}_{i j}$ lie in the range of $[0,1]$. Basic probabilistic assignments, $\bar{m}_{i}(j)$, are fused using Equations 3.4 and 3.5, and a decision of accept or reject is made by transforming the fused bpa into probability measure $(B e t P)$ and then performing the 
likelihood ratio test.

\subsection{SVM Learning based Match Score Fusion Algo- rithm}

The evidence theoretic sum rule does not guarantee handling of non-linear biometric match scores generated from non-ideal images. In literature, non-linear fusion techniques using support vector machine have been proposed to address non-linearity in biometric match scores [1]. However, existing algorithms are computationally complex and parameter estimation is heuristic. In this section, we present a quality based SVM fusion algorithm to combine match scores obtained by matching level-2 and level-3 fingerprint features. The algorithm uses $2 \nu$-SVM for fusion and classification which is a computationally efficient variant of SVM. We first provide a brief overview of $2 \nu$-SVM followed by the proposed quality induced $2 \nu$-SVM match score fusion algorithm.

\subsubsection{Overview of $2 \nu-\mathrm{SVM}$}

In biometrics, support vector machine [107] has been used for different learning based operations such as face recognition [101] and multimodal fusion [1]. SVM starts with the goal of separating the data with a hyperplane and extends it to non-linear decision boundaries. SVM is thus a classifier that performs classification by constructing hyperplanes in a multidimensional space and separating the data points into different classes. To construct an optimal hyperplane, SVM uses an iterative training algorithm which maximizes the margin between two classes. However, some researchers have shown that margin maximization does not always minimize the classification error [20, 81]. 
Sometimes the training data points are not clearly separable and are characterized as fuzzy separable data. In biometrics, poor quality images and images containing noise due to sensor often lead to incorrect classification and hence can be considered as fuzzy data. To address these challenges, we use dual $\nu$-SVM originally proposed by Chew et al. [22]. $2 \nu$-SVM is an attractive alternative to SVM and offers much more natural setting for parameter selection which is a critical issue in practical applications.

Let $\left\{\mathbf{x}_{i}, y_{i}\right\}$ be a set of $N$ data vectors with $\mathbf{x}_{i} \in \Re^{d}, y_{i} \in(+1,-1)$, and $i=1, \ldots, N$. $\mathbf{x}_{i}$ is the $i^{\text {th }}$ data vector that belongs to a binary class $y_{i}$. According to Chew et al. [22], the objective of training $2 \nu$-SVM is to find the hyperplane that separates two classes with the widest margins, i.e.,

$$
\mathbf{w} \varphi(\mathbf{x})+b=0
$$

subject to,

$$
y_{i}\left(\mathbf{w} \varphi\left(\mathbf{x}_{i}\right)+b\right) \geq\left(\rho-\psi_{i}\right), \quad \rho, \psi_{i} \geq 0
$$

to minimize,

$$
\frac{1}{2}\|\mathbf{w}\|^{2}-\sum_{i} C_{i}\left(\nu \rho-\psi_{i}\right)
$$

where $\rho$ is the position of the margin and $\nu$ is the error parameter. $\varphi(\mathbf{x})$ is the mapping function used to map the data space to the feature space and provide generalization for the decision function that may not be a linear function of the training data. $C_{i}\left(\nu \rho-\psi_{i}\right)$ is the cost of errors, $w$ is the normal vector, $b$ is the bias, and $\psi_{i}$ is the slack variable for classification errors. Slack variables are introduced to handle classes which cannot be separated by a hyperplane. $\nu$ is the error parameter that can be calculated using 
Equation 3.14.

$$
\nu=\frac{2 \nu_{+} \nu_{-}}{\nu_{+}+\nu_{-}}, \quad 0<\nu_{+}<1 \text { and } 0<\nu_{-}<1
$$

Here, $\nu_{+}$and $\nu_{-}$are the error parameters for training the positive and negative classes respectively. Error penalty $C_{i}$ is calculated as,

$$
C_{i}=\left\{\begin{array}{lll}
C_{+}, & \text {if } & y_{i}=+1 \\
C_{-}, & \text {if } & y_{i}=-1
\end{array}\right.
$$

where,

$$
\begin{aligned}
& C_{+}=\left[n_{+}\left(1+\frac{\nu_{+}}{\nu_{-}}\right)\right]^{-1} \\
& C_{-}=\left[n_{-}\left(1+\frac{\nu_{-}}{\nu_{+}}\right)\right]^{-1}
\end{aligned}
$$

and $n_{+}$and $n_{-}$are the number of training points for the positive and negative classes respectively. Further, $2 \nu$-SVM training can be formulated as [22],

$$
\max _{\left(\alpha_{i}\right)}\left\{-\frac{1}{2} \sum_{i, j} \alpha_{i} \alpha_{j} y_{i} y_{j} K\left(x_{i}, x_{j}\right)\right\}
$$

where,

$$
\begin{aligned}
& 0 \leq \alpha_{i} \leq C_{i} \\
& \sum_{i} \alpha_{i} y_{i}=0 \\
& \sum_{i} \alpha_{i} \geq \nu
\end{aligned}
$$

$i, j \in 1, \ldots, N, \alpha_{i}, \alpha_{j}$ are the Lagrange multipliers and the kernel function is 


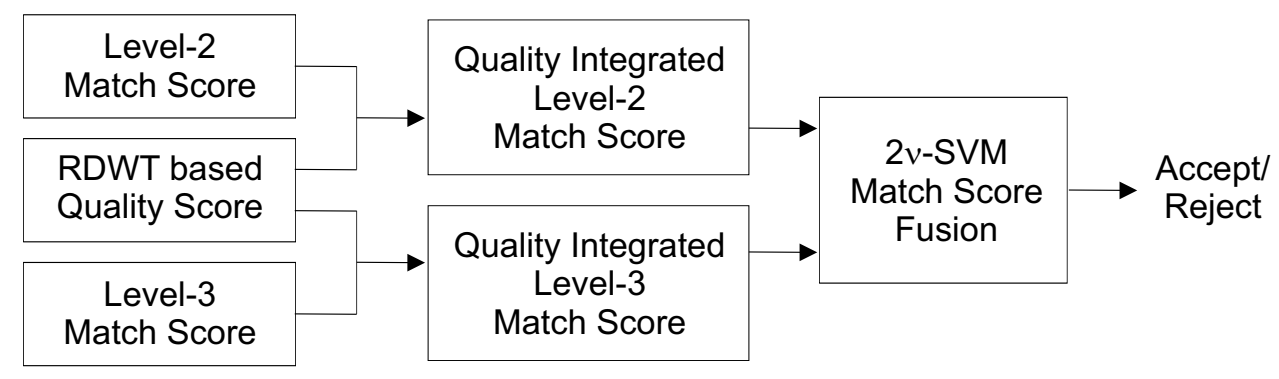

Figure 3.4: Illustrating the steps involved in the $2 \nu$-support vector machine based match score fusion algorithm.

$$
K\left(\mathbf{x}_{i}, \mathbf{x}_{j}\right)=\varphi\left(\mathbf{x}_{i}\right) \varphi\left(\mathbf{x}_{j}\right)
$$

Here we use kernel function $K\left(\mathbf{x}_{i}, \mathbf{x}_{j}\right)$ as the RBF kernel [107]. To train $2 \nu$-SVM, we use the iterative decomposition training based optimization algorithm [22]. This optimization algorithm can be viewed as pairwise decomposition method which breaks the problem to a two variable decision problem and solves the subproblem analytically. Chew et al. [22], have shown that the optimized $2 \nu$-SVM has a complexity of $O(N)$ which is significantly faster than $O\left(N^{2}\right)$ of the classical SVM. Applying the optimization algorithm thus leads to reduction in the computational complexity.

\subsubsection{Fusion of Match Score and Quality Score using $2 \nu$-SVM}

In this section, we describe the proposed $2 \nu$-SVM based fusion algorithm which combines the match score and quality scores for improved recognition performance. The match scores generated from level-2 and level-3 feature matching algorithms are incorporated with the fingerprint quality scores and fused using the proposed $2 \nu$-SVM fusion algorithm (Figure 3.4).

Let $s_{1}$ be the match score for level-2 features and $s_{2}$ be the match score for level-3 
features. Let $q_{m e d}$ be the quality score of the fingerprint image. The product of the quality score with the corresponding match score represents the quality based match score metric, $S q$.

$$
\begin{aligned}
& S q_{1}=q_{\text {med }} \cdot s_{1} \\
& S q_{2}=q_{\text {med }} \cdot s_{2}
\end{aligned}
$$

Quality based match scores and their labels are used to train the $2 \nu$-SVM for multimodal fusion. Let the labeled training data be represented as $Z_{1}=\left(S q_{1}, y\right)$ and $Z_{2}=\left(S q_{2}, y\right)$. For each data, the class label $y \in(+1,-1)$, where +1 represents the genuine class and -1 represents the impostor class. Two $2 \nu$-SVMs are trained using these labeled training data; one for level-2 matcher and another for level-3 matcher. During the training of $2 \nu$-SVM, error parameters $\nu_{+}$and $\nu_{-}$are computed as follows:

$$
\begin{aligned}
& \nu_{+}=\frac{n_{+}}{n_{+}+n_{-}} \\
& \nu_{-}=\frac{n_{-}}{n_{+}+n_{-}}
\end{aligned}
$$

Here $n_{+}$and $n_{-}$are the number of genuine and impostor training data respectively. Training data is mapped in a higher dimension feature space such that $Z \rightarrow \varphi(Z)$ where $\varphi(\cdot)$ is the mapping function. The optimal hyperplane which separates the data into two different classes in the higher dimensional feature space can be obtained as the solution of Equation 3.12.

In the testing phase, quality based fused score $f\left(S q_{f u s e d}\right)$ of a multimodal test pattern $\left[S q_{1}, S q_{2}\right]$ is defined as, 


$$
f\left(S q_{\text {fused }}\right)=f_{1}\left(S q_{1}\right)+f_{2}\left(S q_{2}\right)
$$

where,

$$
\begin{aligned}
& f_{1}\left(S q_{1}\right)=w_{1} \varphi\left(S q_{1}\right)+b_{1} \\
& f_{2}\left(S q_{2}\right)=w_{2} \varphi\left(S q_{2}\right)+b_{2}
\end{aligned}
$$

Here, $w_{1}, w_{2}, b_{1}$, and $b_{2}$ are the parameters of the hyperplane. The solution of Equation 3.25 is the signed distance of $S q_{\text {fused }}$ from the separating hyperplane given by the two $2 \nu$-SVMs. Finally, to verify the identity, a decision to accept or reject is made on the test pattern $S q_{\text {fused }}$ as follows,

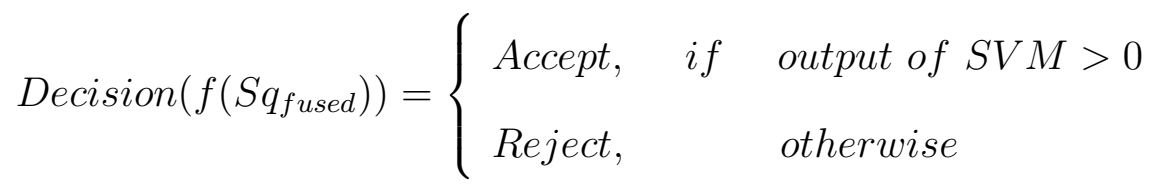

\subsection{Proposed Proportional Conflict Redistribution Rule based Match Score Fusion Algorithm}

A major problem with statistical and learning based multibiometric fusion algorithms occurs when different biometric classifiers generate highly conflicting results. Specifically, if one classifier strongly supports one hypothesis and the other classifier strongly rejects the same hypothesis. For example, in a multiclassifier fingerprint recognition system, variance in image capture, image quality, and sensor noise could generate a level-2 match score of 0.8 (perfect accept is 1) and a level-3 match score of 0.2 (perfect reject is 0). Existing fusion algorithms may not be able to handle such conflicting 
information thus affecting the verification performance. In this section, we describe a belief function theory based match score fusion algorithm that estimates the genuine and impostor densities using generalized basic belief assignment and performs fusion using PCR rule. This section first presents an overview of belief function theories followed by the proposed PCR fusion algorithm.

\subsubsection{Overview of Belief Function Theory based Fusion Algo- rithms}

Belief function theory or theory of evidence is a theoretical framework for reasoning with imperfect data. It is a generalization of probability theory and includes many approaches of reasoning under uncertainty. Examples of such approaches are Dempster Shafer theory, Transferable Belief Model, Dezert Smarandache fusion, and Proportional Conflict Redistribution rule. In this section, only the main concepts and notations of Dempster Shafer theory, Transferable Belief Model, Dezert Smarandache fusion, and PCR rule are presented for a two class - two classifier problem. A detailed explanation of belief function theory can be found in $[26,82,91,111]$.

Probability theory based evidence theoretic sum rule is the basis of belief function theory. The basic sum rule fusion, though effective for simple non-conflicting cases, is not very effective for imprecise, uncertain, and conflicting cases. To address the limitations of evidence theoretic sum rule, approximate reasoning approach based fusion rules including DS theory, TBM and DSm fusion have been proposed.

Let $m \in[0,1]$ be a mapping function and $\Theta=\left\{\theta_{1}, \theta_{2}\right\}$ be the frame of discernment that represents the finite set of exhaustive and mutually exclusive hypothesis. In DS theory, belief functions are computed on the power set of $\Theta$ (i.e. $2^{\Theta}$ ) and Dempster's 
rule of combination $[82,114]$ for fusing two information sources, $X$ and $Y$, is defined as,

$$
m_{D S}(A)=\frac{\sum_{\left(X, Y \in 2^{\Theta}\right),(X \cap Y=A)} m(X) m(Y)}{1-\sum_{\left(X, Y \in 2^{\Theta}\right),(X \cap Y=\emptyset)} m(X) m(Y)}
$$

Although DS theory based fusion has been efficiently used for many practical applications, it has some limitations. As presented by Zadeh [116], Dubois and Prade [30], Voorbraak [111], and Dezert and Smarandache [26], DS theory is not reliable when conflict between the sources is very large. To circumvent the limitations of DS fusion algorithm, researchers have proposed several other models. Smets proposed transferable belief model [91] that quantitatively represents the epistemic uncertainty. According to Smets, the TBM conjunctive combination rule for fusing two information sources, $X$ and $Y$, can be represented as,

$$
m_{T B M}(A)=\sum_{X, Y \in 2^{\Theta}} m(X) m(Y)
$$

Recently, Dezert and Smarandache proposed a fusion algorithm using plausible and paradoxical reasoning [26] that addresses the limitations of DS theory and includes Bayes theory and DS theory as special cases. It operates on the hyperpower set defined as $D^{\Theta}=\left\{\emptyset, \theta_{1}, \theta_{2}, \theta_{1} \cup \theta_{2}, \theta_{1} \cap \theta_{2}\right\}$. This algorithm uses generalized basic belief assignment on $\Theta$ which is defined as $m(\cdot): D^{\Theta} \rightarrow[0,1]$ such that

$$
\begin{aligned}
& m(\emptyset)=0 \\
& m\left(\theta_{1}\right)+m\left(\theta_{2}\right)+m\left(\theta_{1} \cup \theta_{2}\right)+m\left(\theta_{1} \cap \theta_{2}\right)=1
\end{aligned}
$$

For fusing two information sources, $X$ and $Y$, the DSm rule of combination [26] is defined as,

$$
m_{M(\Theta)}(A)=\psi(A)\left[S_{1}(A)+S_{2}(A)+S_{3}(A)\right]
$$


where, $M(\Theta)$ is the model over which DSm theory operates and $\psi(A)$ is the characteristic non-emptiness function of $A$ which is 1 if $A \notin \emptyset$ and 0 otherwise. $S_{1}(A), S_{2}(A)$, and $S_{3}(A)$ are defined as,

$$
\begin{array}{ll}
S_{1}(A)=\sum_{\left(X, Y \in D^{\Theta}, X \cap Y=A\right)} & m_{1}(X) m_{2}(Y) \\
S_{2}(A)=\sum_{\left(X, Y \in \boldsymbol{\Phi},[v=A] \vee\left[(v \in \boldsymbol{\Phi}) \wedge\left(A=I_{t}\right)\right]\right)} & m_{1}(X) m_{2}(Y) \\
S_{3}(A)=\sum_{\left(X, Y \in D^{\Theta}, X \cup Y=A, X \cap Y \in \boldsymbol{\Phi}\right)} & m_{1}(X) m_{2}(Y)
\end{array}
$$

where $I_{t}$ is the total ignorance and is the union of all $\theta_{i}(i=1,2)$, i.e. $I_{t}=\theta_{1} \cup \theta_{2}$. $\boldsymbol{\Phi}=\{\boldsymbol{\Phi}, \phi\}$ is the set of all elements of $D^{\Theta}$ which are empty under the constraints of some specific problem and $\phi$ is the empty set. $v=u(X) \cup u(Y)$, where $u(X)$ is the union of all singletons $\theta_{i}$ that compose $X$ and $Y$. Here, $S_{1}(A)$ corresponds to the classical DSm rule on the free DSm model [26], $S_{2}(A)$ represents the mass of all relatively and absolutely empty sets which is transferred to the total or relative ignorance, and $S_{3}(A)$ transfers the sum of relative empty sets to the non-empty sets.

In DSm fusion algorithm, the partial conflicts are redistributed onto corresponding partial ignorance [26]. However, in some cases this redistribution may yield very nonspecific generalized basic belief assignments and thus decrease the performance. Further analysis by Smets [93] suggests that the term $S_{2}$ in Equation 3.33 is a potential source of difficulties and seems to be language dependent. To address this issue, Dezert and Smarandache proposed set of proportional conflict redistribution rules [27] that consists of five different versions of the PCR rule; PCR1 to PCR5 in order of increasing complexity and correctness. They have reported that among the five rules, PCR5 is the most efficient and precise for information fusion under uncertainty and conflict. In PCR5, redistribution of the partial conflicts is performed only to the elements which are truly involved in each partial conflict and this is done according to the proportion or weight of each source. For 
a two-class, two-classifier problem and $\forall X \in D^{\Theta} \backslash\{\emptyset\}$, PCR5 rule [27] is defined as

$$
m_{P C R 5}(X)=m_{12}(X)+\sum_{Y \in D^{\Theta} \backslash\{X\}, \bar{c}(X \cap Y)}\left[\frac{m_{1}(X)^{2} m_{2}(Y)}{m_{1}(X)+m_{2}(Y)}+\frac{m_{2}(X)^{2} m_{1}(Y)}{m_{2}(X)+m_{1}(Y)}\right]
$$

In this equation, $m_{1}$ and $m_{2}$ represent the corresponding belief assignments of the two classifiers. $m_{12}(X)$ represents the conjunctive rule as presented in Equation 3.30 and $\bar{c}(\cdot)$ represents the canonical form (Equation 3.35).

$$
\begin{aligned}
& \text { If } \quad X=(A \cap B) \cap(A \cup B \cup C) \\
& \text { then } \bar{c}(X)=(A \cap B)
\end{aligned}
$$

PCR5 fusion rule precisely combines and redistributes the information even with conflicting gbbas. A detailed explanation of PCR rule can be found in [27].

\subsubsection{PCR5 based Biometric Match Score Fusion Algorithm}

One of the major problems with multimodal biometrics is with unbalanced systems where two different classifiers have different accuracies and sometimes have uncertain and highly conflicting results. We propose to apply the plausible and paradoxical reasoning of PCR rule for fusing biometric information. Furthermore, we associate quality score of the input image to increase robustness of the fusion algorithm. In this section, we describe the proposed PCR rule based multimodal biometric fusion algorithm using image quality scores.

Figure 3.5 illustrates the steps involved in the match score fusion algorithm which utilizes PCR5 rule [26, 89] to combine the outputs of level-2 and level-3 fingerprint verification algorithms. Fingerprint verification algorithms described in $[48,55]$ are used to obtain match scores corresponding to level-2 features and match score for level-3 


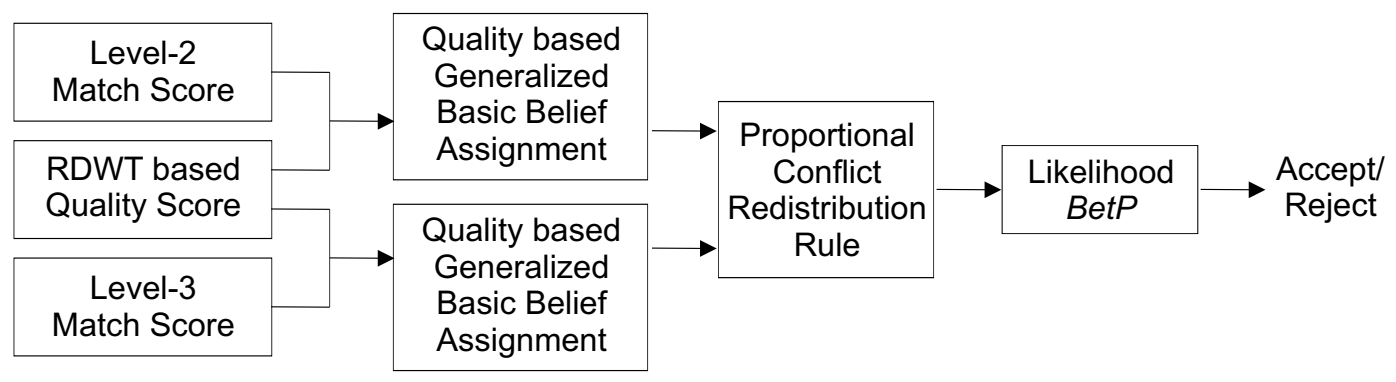

Figure 3.5: PCR rule based fusion of quality-augmented match scores obtained from level-2 and level-3 fingerprint verification algorithms.

features are obtained using the algorithm described in Chapter 2. Let the matching score generated by the classifiers be $s_{j}$ where $j=1,2$ represents the fingerprint classifiers. The quality score $q_{m e d}$, median value of quality score vector, computed in Section 2.1.1 is augmented with the matching score $s_{j}$ to generate the quality-augmented match score $S q_{j}$

$$
S q_{j}=q_{m e d} \cdot s_{j}
$$

In the proposed PCR match score fusion algorithm, we define the frame of discernment, $\Theta=\left\{\theta_{\text {gen }}, \theta_{\text {imp }}\right\}$ and Dedekind lattice, $D^{\Theta}=\left\{\theta_{\text {gen }}, \theta_{\text {imp }}, \theta_{\text {gen }} \cup \theta_{\text {imp }}, \theta_{\text {gen }} \cap \theta_{\text {imp }}\right\}$. Further, for every input fingerprint image, each classifier assigns a label true or 1 to proposition $a, a \in \Theta$ and the remaining classes are labeled as false or 0 . Thus, there are two focal elements for each fingerprint verification algorithm, $a$ and $\neg a(\neg a=\Theta-a)$. $a$ is for confirming and $\neg a$ is for denying a proposition for mass assignment in the PCR. For every verification algorithm or classifier, we compute the respective predictive rates which are used to assign their $g b b a$. Let an input pattern belonging to class $i\left(i \in D^{\Theta}\right)$ be classified as one of the $k\left(k \in D^{\Theta}\right)$ classes. Then, the predictive rate [92] of a classifier $P_{k}$ for an output class $k$ is the ratio of the number of input patterns classified correctly 
to the total number of patterns classified as class $k$ where input patterns belonging to all classes is presented to the classifier.

In the proposed PCR rule based approach, when the $j^{\text {th }}$ fingerprint verification algorithm classifies the result $k \in D^{\Theta}$ over the normalized matching score $S q_{j}$, it is considered that for all instances the likelihood of $k$ being the actual class is $P_{k j}$ and the likelihood of $k$ not being the correct class is $\left(1-P_{k j}\right)[92]$. For the $j^{\text {th }}$ fingerprint verification algorithm, the generalized basic belief assignment or mass $m_{j}(k)$ is computed using Equation 3.37 by multiplying $P_{k j}$ with the quality-augmented normalized match score $S q_{j}$.

$$
m_{j}(k)=P_{k j} \cdot S q_{j}
$$

Here $j=1,2$ corresponds to the two fingerprint verification algorithms. Similarly, the disbelief is assigned to $m_{j}(\neg k)$ with $m(\Theta)=1$. Further, the mass of each evidence or classifier is combined to generate the generalized basic belief assignment of the fused information, $m_{\text {final }}$ using Equation 3.38,

$$
m_{\text {final }}=m_{1} \oplus m_{2}
$$

where $\oplus$ represents the PCR5 fusion rule defined in Equation 3.34. The final verification result is obtained by applying threshold $\mathbf{t}$ to $m_{\text {final }}$,

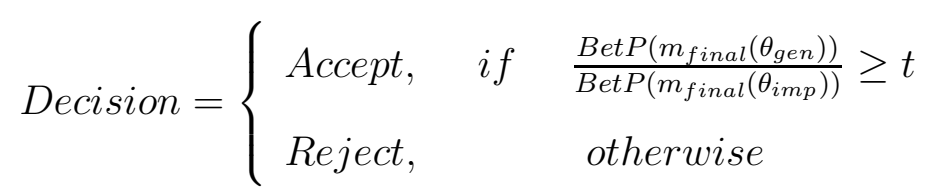




\subsection{Proposed Sequential Match Score Fusion Algo- rithm}

Previous sections present match score fusion algorithms using the density approach, the classifier learning approach, and the belief model approach. In the research on match score fusion, we observe that,

1. Density-based or statistical fusion schemes which use the likelihood ratio test to formulate the fusion rule are impacted by incorrect estimates of the density functions pertaining to the genuine and impostor scores. The use of parametric methods of density estimation is often based on the assumption of incorrect models (e.g., Gaussian densities for both genuine and impostor scores) that can lead to sub-optimal fusion rules; the use of non-parametric methods is detracted by the availability of a small number of training samples (especially genuine scores) thereby impacting the feasibility of designing an effective fusion rule.

2. Classifier-based fusion schemes, on the other hand, are too rigid and often lead to a transductive type of learning that cannot handle conflicting match scores effectively.

3. Belief model based fusion schemes are computationally complex and ad-hoc in nature.

In this section, a sequential fusion algorithm is proposed in which belief model based fusion approach is combined with statistical and learning approaches. Its novelty lies in incorporating the density-based fusion scheme into a belief model and the use of likelihood ratio test-statistic with a support vector machine classifier. The density estimation and likelihood ratio aspect of the algorithm makes it robust to uncertainties in the component 


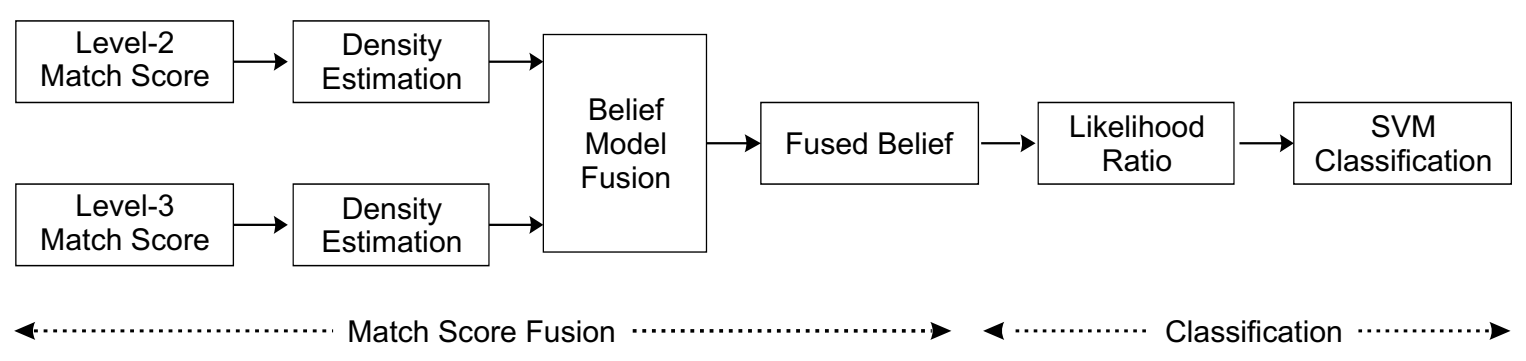

Figure 3.6: Proposed sequential match score fusion algorithm.

matchers; the use of belief model makes it robust to conflicting cases, and the use of Support Vector Machine ensures that the algorithm is less prone to over-fitting thereby permitting it to handle non-linear biometric information. Further, utilizing the belief model for fusion and SVM for classification addresses the limitations of small sample size density estimation for conflicting cases.

Figure 3.6 shows the steps involved in the proposed fusion algorithm that consists of two steps: (1) match score fusion and (2) classification. First, the match scores are transformed into belief assignments using density estimation technique. In the next step, belief model is used for fusion and finally, statistical likelihood ratio and SVM are used for classification. The fusion algorithm uses a two-class, two-classifier approach.

\subsubsection{Sequential Match Score Fusion}

For a two class problem, let $\Theta=\left\{\theta_{\text {gen }}, \theta_{\text {imp }}\right\}$, where $\theta_{\text {gen }}$ represents the genuine hypothesis and $\theta_{\text {imp }}$ represents the impostor hypothesis. The first step in the sequential fusion algorithm is to transform match scores into belief assignments. Since the belief functions are a generalized form of probability theory that can perform fusion in presence of uncertainty and imperfect data, probabilistic approach can be effectively used as the basis of the fusion algorithm. A multivariate density estimation technique is used to compute belief assignments induced by the match scores because previous literature has 
shown the usefulness of mixture models in biometrics [67]. Multivariate Gaussian density [31] in $d$ dimensions can be written as,

$$
p(\mathbf{s}, \mu, \boldsymbol{\Sigma})=\frac{1}{(2 \pi)^{d / 2}|\mathbf{\Sigma}|^{1 / 2}} \exp \left[-\frac{1}{2}(\mathbf{s}-\mu)^{t} \mathbf{\Sigma}^{-1}(\mathbf{s}-\mu)\right]
$$

where $\mathbf{s}$ is a vector with $d$ components, $\mu$ is the mean vector, and $\boldsymbol{\Sigma}$ is the covariance matrix. Let $C_{n}(\mathbf{s}, i)$ be the conditional joint density of $d$ match scores obtained from the $n^{\text {th }}$ classifier and $i \in \Theta . C_{n}(\mathbf{s}, i)$ is computed using Equation 3.41.

$$
C_{n}(\mathbf{s}, i)=\sum_{j=1}^{M_{n}(i)} W_{n}(i, j) p\left(\mathbf{s}, \mu_{n}(i, j), \boldsymbol{\Sigma}_{n}(i, j)\right)
$$

where $\mu_{n}(i, j), \boldsymbol{\Sigma}_{n}(i, j)$ and $W_{n}(i, j)$ are the mean vector, covariance matrix, and weight factor respectively corresponding to the $j^{\text {th }}$ mixture component in the conditional joint density. Also, $\sum_{j=1}^{M_{n}(i)} W_{n}(i, j)=1$ and $M_{n}(i)$ is the number of mixture components used to model the density. Further, a recursive algorithm [119] is used to estimate the parameters of the mixture model.

Let $\mathbf{s}=\left(s_{1}, s_{2}\right)$ be the match scores computed from two biometric classifiers or matchers. Belief assignment of the $n^{\text {th }}$ classifier, $m_{n}$, is computed using Equation 3.42.

$$
m_{n}(i)=\frac{\alpha_{n}(i) C_{n}\left(s_{n}, i\right)}{\sum_{i} \alpha_{n}(i) C_{n}\left(s_{n}, i\right)}
$$

where $C_{n}\left(s_{n}, i\right)$ is the marginal density and $\alpha_{n}(i)$ is the verification accuracy prior of the $n^{\text {th }}$ classifier that is used as the ancillary information to attune the beliefs. With the help of Equation 3.42, the belief assignments of each biometric classifier are computed. For example, in a two classifier biometric system, we compute $\left\{m_{1}\left(\theta_{\text {gen }}\right), m_{1}\left(\theta_{\text {imp }}\right)\right\}$ and $\left\{m_{2}\left(\theta_{\text {gen }}\right), m_{2}\left(\theta_{\text {imp }}\right)\right\}$.

The belief assignments of each biometric classifier are then fused using the pro- 
portional conflict redistribution rule [27]. In this rule, redistribution of the conflicts is performed only to the elements which are involved in each conflict and is done according to the proportion/weight of each classifier. The belief assignments of classifier $c_{1}$ and $c_{2}$ are fused using Equation 3.43.

$$
m_{\text {fused }}(i)=m_{c_{1}}(i) m_{c_{2}}(j)+w_{1} \frac{m_{c_{1}}^{2}(i) m_{c_{2}}(j)}{m_{c_{1}}(i)+m_{c_{2}}(j)}+w_{2} \frac{m_{c_{2}}^{2}(i) m_{c_{1}}(j)}{m_{c_{2}}(i)+m_{c_{1}}(j)}
$$

Here $i, j \in \Theta, i \neq j$, and $w_{1}$ and $w_{2}$ are the weight factors. $m_{1}$ and $m_{2}$ denote the belief assignments of level-2 feature based matcher and level-3 feature based matcher respectively computed using Equation 3.42. $\mathbf{m}_{\text {fused }}$ is the fused belief and is a vector with values $\left\{m_{\text {fused }}\left(\theta_{\text {gen }}\right), m_{\text {fused }}\left(\theta_{\text {imp }}\right)\right\}$. In Equation 3.43, the first term denotes the degree of conflict between the classifiers and the formulation effectively combines the beliefs of multi-classifier match scores even with conflicts. Further, to incorporate quality score in the sequential fusion algorithm, we compute joint densities of the match scores and quality scores estimated from the genuine and impostor gallery-probe pairs, and use it in belief assignment (Equation 3.42) and then in fusion.

\subsubsection{Classification}

For decision making or classification, first the fused belief assignments induced from

match scores are converted into the likelihood ratio $R=\frac{m_{\text {fused }}\left(\theta_{\text {gen }}\right)}{m_{\text {fused }}\left(\theta_{\text {imp }}\right)}$. Next, the likelihood ratio is used as input to the SVM classifier for decision making (Equation 3.44). Utilizing the SVM with likelihood ratio for decision making addresses the non-linearity in the biometric match scores. 


$$
\text { Decision }= \begin{cases}\text { accept } & \text { if } \quad \operatorname{SVM}(R) \geq t \\ \text { reject } & \text { otherwise }\end{cases}
$$

Here $t$ is the decision threshold chosen for a specific false accept rate. The advantage of this approach is its control over the false accept and false reject rates, and it also satisfies the Neyman-Pearson theorem [63] for decision making.

\subsection{Experimental Results}

The five proposed fusion algorithms are evaluated on the high resolution fingerprint databases explained in Chapter 2. The first database is augmented with the non-ideal database such that the number of classes in the joint database is 710 with 10 fingerprint images per class. Four images from each class are randomly selected for training and the remaining images are selected for testing (gallery-probe matching). This protocol provides 2840 images for training and 4260 images for testing. This train-test partitioning is repeated 20 times for cross validation. Finally, ROC curves are generated and verification accuracies are reported at $0.01 \%$ FAR. With 710 classes, we compute verification accuracies of level-2 and level-3 feature matching. This provides a baseline for fusion algorithms to show the improvement in verification performance. We also compare the performance with existing algorithms namely sum rule [76], classical SVM fusion [1] and product of likelihood ratio fusion [67].

Level-2 and level-3 feature matching algorithms provide accuracy of $85.6 \%$ and $87.5 \%$ respectively. Match score fusion of level-2 features and level-3 features using the proposed fusion algorithms improves the verification accuracy in the range of $6.7-10.6 \%$. Figures 3.7-3.10 show the ROC plots for the fusion algorithms and Table 3.1 summarizes 
the verification accuracies. Analysis of the key results are described below:

1. Evidence theoretic sum rule yields an accuracy of $94.2 \%$ which is $1.7 \%$ better than classical sum rule [76]. This is because the evidence theoretic sum rule incorporates image quality score and verification prior, and the decision making process incorporates likelihood ratio test statistics.

2. In experiments with $2 \nu$-SVM fusion, we evaluate the performance of the proposed fusion algorithm with three variants of Support Vector Machine namely, SVM [107], $\nu$-SVM [20], and $2 \nu$-SVM [22]. Further, for each of the three SVMs, we also evaluate the verification performance of the fusion algorithms with three kernels: linear, polynomial, and Radial Basis Function. This experiment is performed to justify the choice of $2 \nu$-SVM and RBF kernel in the proposed $2 \nu$-SVM match score fusion algorithm. The optimal parameters for the SVMs and the kernels are obtained empirically by computing the verification accuracy for different combination of parameters. Table 3.2 shows the results obtained for optimal parameters. For $2 \nu$-SVM based fusion, optimal parameters corresponding to the polynomial kernel are $r=1$, $\gamma=1$, and $d=2$ and for RBF kernel $\gamma=4$. The results show that for all three SVMs, non-linear kernels provide higher verification performance compared to the linear kernel. This is because biometric match scores are non-linearly distributed and hence non-linear kernels provide better classification performance. Table 3.2 further shows that with optimal parameters, $2 \nu$-SVM with RBF kernel provides the best verification performance of $95.6 \%$.

3. The ROC plots in Figure 3.8 show that the performance of the proposed $2 \nu$-SVM fusion algorithm is better than C-SVM fusion algorithm [1]. This is mainly because of the use of the proposed RDWT based quality assessment algorithm, $2 \nu$-SVM and 
RBF kernel.

4. The proposed PCR5 rule based belief model fusion algorithm yields the verification accuracy of $96.7 \%$ and improves the recognition performance by at least $9.2 \%$. We also compare the performance of PCR5 rule with other belief function theory based fusion rules such as DS theory, TBM, and DSm. We observed that the PCR5 rule is computationally fast and yields better accuracy compared to other belief function theory based fusion algorithms even when the individual biometric classifiers provide highly conflicting match scores.

5. In comparison to level-2 and level-3 features, Delaunay triangulation fusion algorithm improves the verification accuracy by at least $8.9 \%$. As shown in Table 3.1, the Delaunay triangulation based fusion is better than evidence theoretic sum rule and $2 \nu$-SVM match score fusion as the Delaunay triangulation fusion algorithm computes additional discriminative fingerprint features and classifies them using quality-induced likelihood ratio based decision algorithm.

6. The sequential fusion algorithm yields an accuracy of $98.1 \%$ which is the best among other fusion algorithms. It effectively combines the properties of statistical, learning and belief theories, and improves the verification accuracy significantly. The algorithm transforms the match scores into probabilistic entity thereby making it robust to sensor noise and matcher limitations. Multiclassifier match score fusion is performed using the proportional conflict redistribution rule that can handle uncertainties in the biometric match scores. Finally, a decision is made using likelihood ratio induced SVM classifier that satisfies the Neyman-Pearson theorem [63].

7. Further, $t$-test [28] at $95 \%$ confidence shows that the sequential fusion algorithm is 
significantly different compared to the level-2 matcher, level-3 matcher, and other fusion algorithms. It is also observed that, according to $t$-test, the five proposed fusion algorithms are significantly different from each other.

8. If both classifiers accept a genuine user, as shown in Figure 3.11, all the fusion rules provide correct results. As shown in Figure 3.12, when conflict between classifiers is not large, all the fusion algorithms correctly classify the genuine user. Further, Figure 3.13 shows a sample case when the two classifiers are in conflict but the proposed sequential fusion algorithm correctly accepts the subject while other fusion algorithms provide incorrect results. Finally, there are few cases (as shown in Figure 3.14) when both the classifiers reject the genuine subject. In such cases, the fusion algorithms cannot reconcile the difference, resulting in incorrect classification.

9. We also evaluate the average time for verification using different fusion algorithms on a $2 \mathrm{GHz}$ Pentium Duo Core processor with 2 GB RAM under MATLAB environment. Among all the fusion algorithms, the classical sum rule is the fastest with 8 seconds that includes level-2 and level-3 feature extraction, matching, fusion, and decision making. On the other hand, the proposed sequential fusion algorithm requires 12 seconds. However, Table 3.1 shows that with only minimal increase in computational time, the accuracy is significantly improved.

\subsection{Summary}

Match scores obtained from level-2 and level-3 matchers show limited correlation and therefore fusion algorithms can be used to improve the verification performance. This 


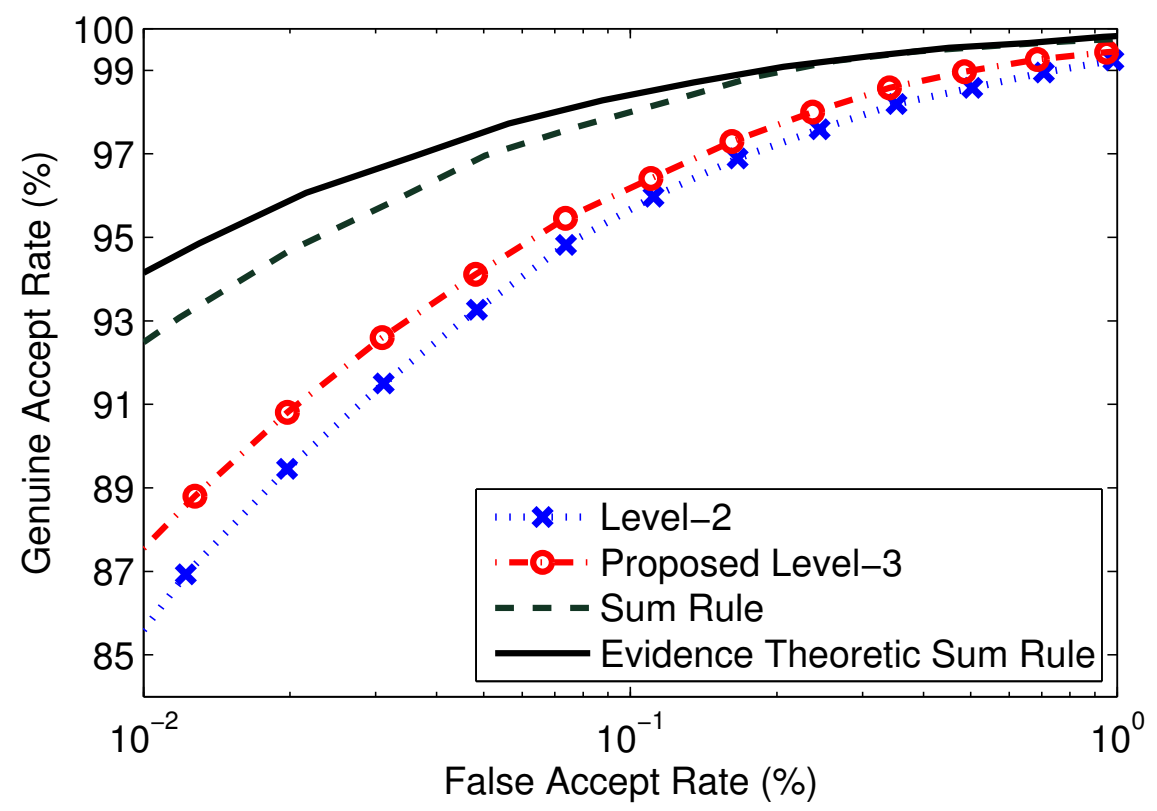

Figure 3.7: ROC plot to evaluate the performance of the proposed evidence theoretic sum rule and comparison with the classical sum rule.

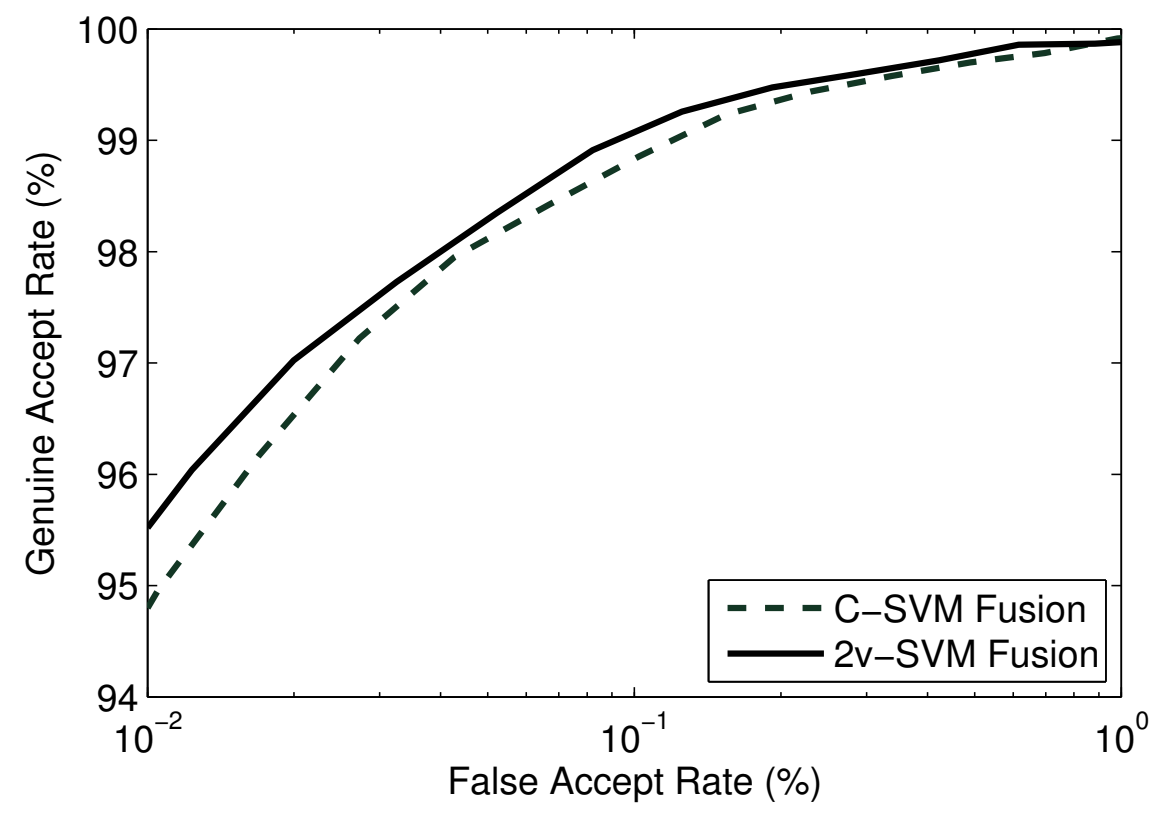

Figure 3.8: ROC plot to compare the performance of the proposed $2 \nu$-SVM match score fusion algorithm with classical SVM fusion algorithm [1]. 
Table 3.1: Verification performance of the proposed fusion algorithms using level-2 and level-3 fingerprint features and comparing with existing match score fusion algorithms. Verification accuracy is computed at $0.01 \%$ False Accept Rate.

\begin{tabular}{|l|c|c|}
\hline Algorithms & $\begin{array}{c}\text { Verification } \\
\text { Accuracy (\%) }\end{array}$ & $\begin{array}{c}\text { Time } \\
\text { (seconds) }\end{array}$ \\
\hline Level-3 Features & 87.5 & 5 \\
\hline Level-2 Features [48], [55] & 85.6 & 3 \\
\hline Sum Rule Fusion [76] & 92.5 & 8 \\
\hline Proposed Evidence-Theoretic Sum Rule & 94.2 & 9 \\
\hline Proposed Delaunay Triangulation & 96.4 & 12 \\
\hline C-SVM Fusion [1] & 94.8 & 10 \\
\hline Proposed 2 $\nu$-SVM Fusion & 95.6 & 10 \\
\hline DST Fusion & 95.2 & 12 \\
\hline TBM Fusion & 95.8 & 12 \\
\hline DSm Fusion & 96.3 & 15 \\
\hline Proposed PCR5 Fusion & 96.7 & 11 \\
\hline Product of Likelihood Ratio & 96.6 & 9 \\
\hline Proposed Sequential Fusion & 98.1 & 12 \\
\hline
\end{tabular}

Table 3.2: Verification accuracy of different SVMs and kernels at 0.01\% FAR.

\begin{tabular}{|c|c|c|c|}
\hline \multirow{2}{*}{$\begin{array}{c}\text { Support Vector } \\
\text { Machines }\end{array}$} & \multicolumn{3}{|c|}{ Verification Accuracy (\%) } \\
\cline { 2 - 4 } & Linear & Polynomial & RBF \\
\hline SVM & 94.9 & 95.1 & 95.1 \\
\hline$\nu$-SVM & 95.0 & 95.1 & 95.3 \\
\hline $2 \nu$-SVM & 95.2 & 95.3 & $\mathbf{9 5 . 6}$ \\
\hline
\end{tabular}




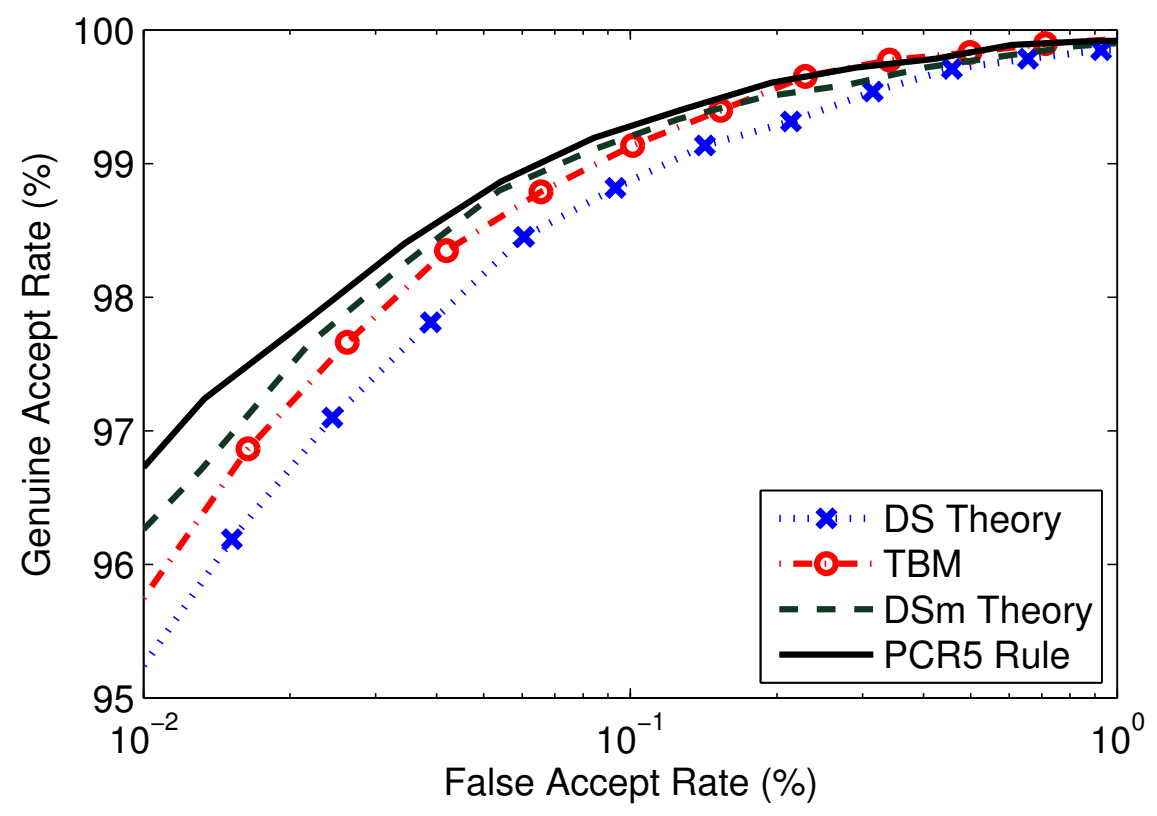

Figure 3.9: ROC plot to compare the performance of the proposed PCR5 match score fusion algorithm with other belief models.

chapter presents five match score fusion algorithms to combine the match scores, namely Delaunay triangulation fusion, evidence theoretic sum rule, $2 \nu$-SVM, PCR5 rule, and sequential fusion. Among the proposed algorithms, sequential fusion algorithm provides the best performance because it leverages the benefits of statistical, learning, and belief theory paradigms. It provides correct results even when the classifiers are in conflict and improves the performance. 


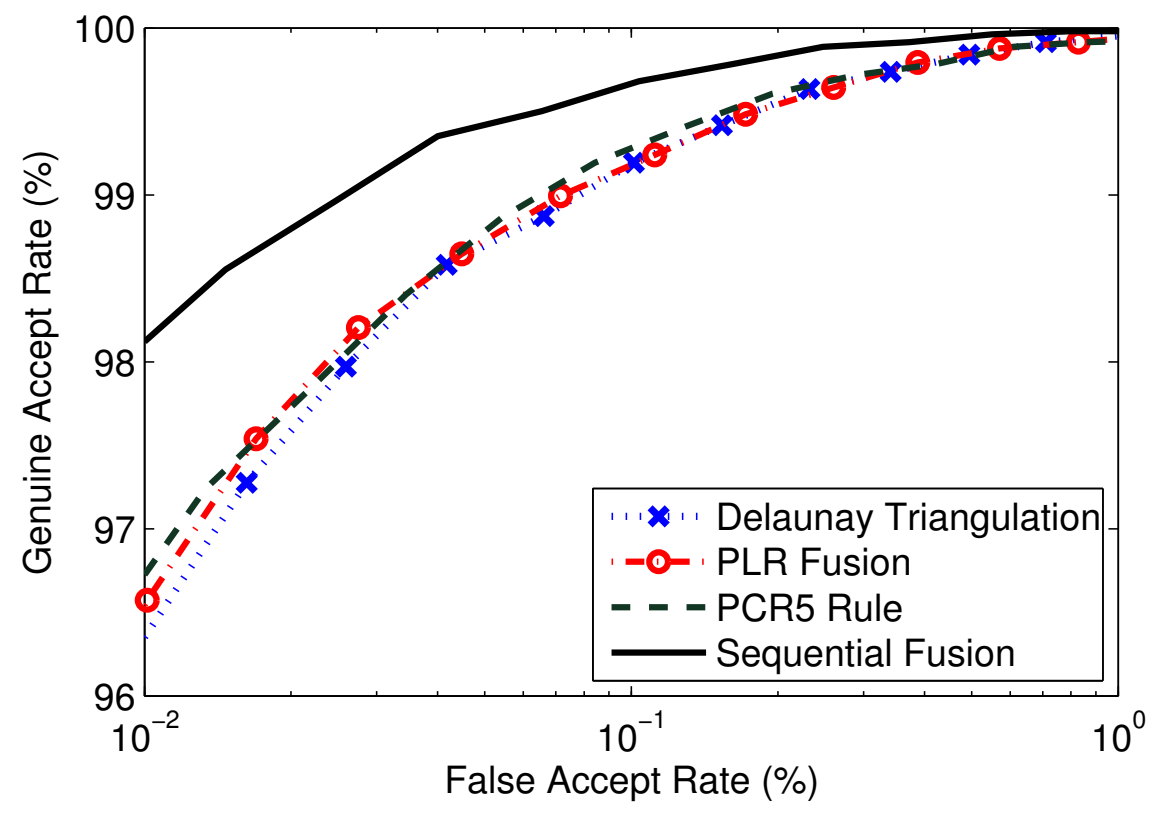

Figure 3.10: ROC plot to compare the performance of the proposed sequential match score fusion algorithm, Delaunay triangulation fusion algorithm, PCR5 rule, and product of likelihood ratio fusion [67].
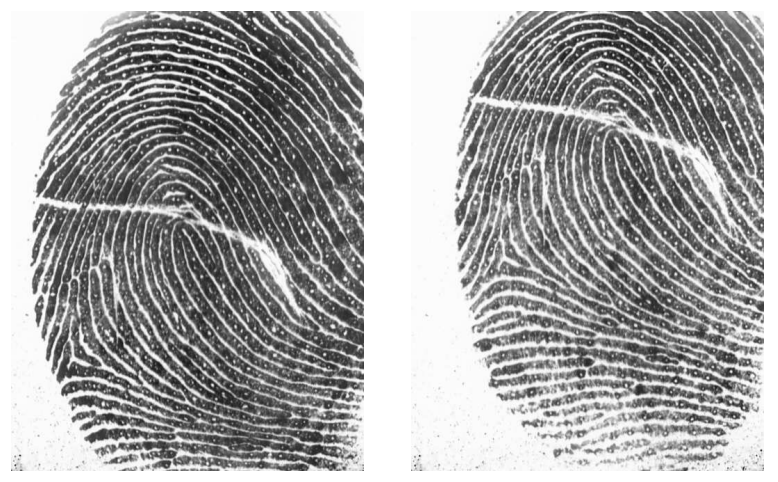

Figure 3.11: Sample gallery-probe pair when both level-2 and level-3 matchers accept the genuine user. 

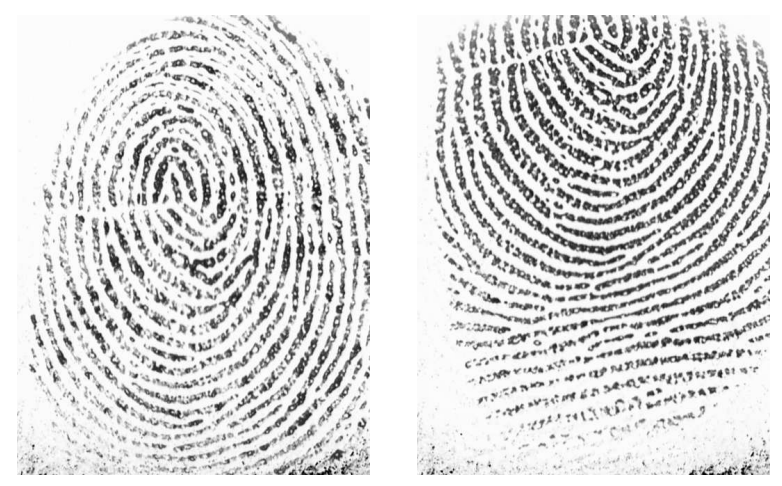

Figure 3.12: Sample gallery-probe pair when level-2 and level-3 matchers are in conflict but all the fusion algorithms accept the genuine user.
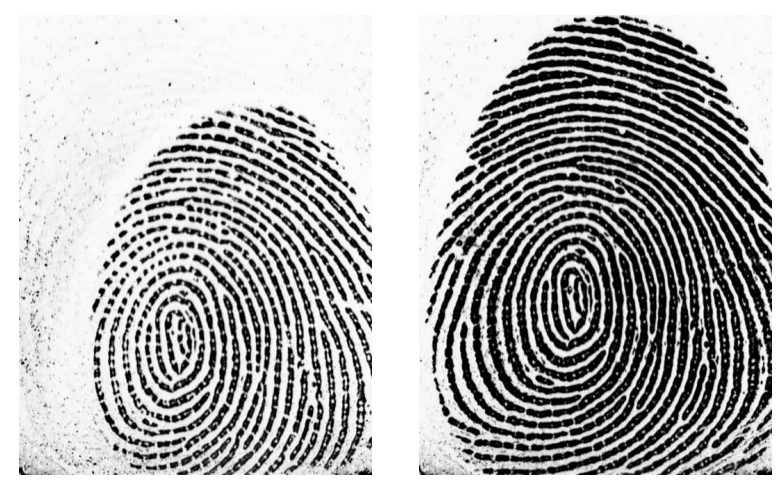

Figure 3.13: Sample gallery-probe pair when level-2 matcher rejects the genuine user but level-3 matcher accepts. The proposed sequential fusion algorithm accepts the user whereas other fusion algorithm rejects.
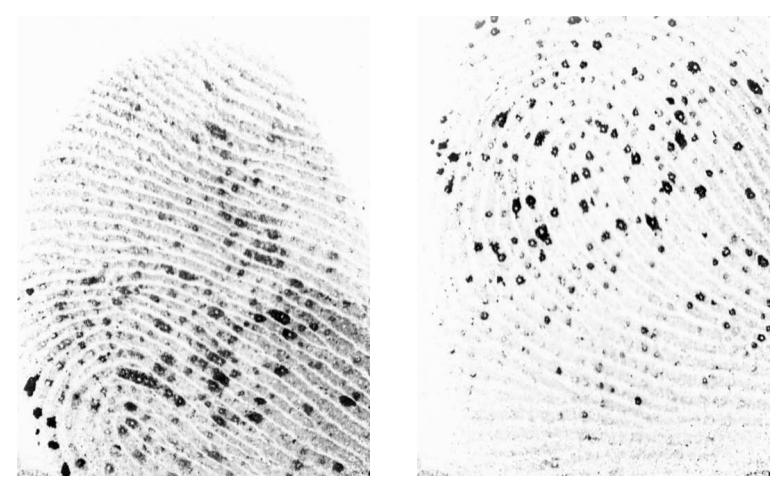

Figure 3.14: Sample gallery-probe pair when both level-2 and level-3 matchers rejects the genuine user. In such cases, fusion algorithms can not provide correct results. 


\section{Chapter 4}

\section{Unification of Fusion algorithms}

Existing biometric fusion algorithms such as sum rule [76] and support vector machine fusion [87] yield good performance for some applications or under certain conditions but not universally for all scenarios. Furthermore, the performance of existing match score fusion algorithms decreases when biometric classifiers yield highly conflicting results. For example, if one biometric classifier generates a match score which corresponds to perfect accept and another classifier provides a match score which corresponds to perfect reject for the same individual, existing fusion algorithms are not able to efficiently perform fusion and matching. The results in Chapter 3 show that the evidence theoretic sum rule is very efficient in terms of time complexity but does not provide good performance when dealing with conflicting cases. Further, the sequential fusion algorithm efficiently combines conflicting results at the expense of higher time complexity. The fusion algorithms are thus unable to fulfill all the requirements of a real world biometric system and do not provide optimal performance for all scenarios. To address this issue, Veeramachaneni et al. [110] proposed an adaptive multimodal decision fusion algorithm using particle swarm optimization to adaptively combine the decision fusion rules and improve the recognition accuracy. Some commercial products also use ad-hoc techniques to select AND and OR rules depending on the desired security level. However, the com- 
plexity of optimizing the algorithm, the limitation of fusion algorithms, and incorporating priors are research challenges that still need to be addressed.

In this chapter, we propose an unification framework to efficiently address both accuracy and time complexity of multimodal biometric fusion. Our hypothesis is that unification or reconciliation of multiple fusion algorithms should satisfy most of the application requirements and yield better recognition performance. Inspired from Smarandache's theoretical concept [90] and Woods et al. research on dynamic classifier selection [112], the unification framework includes a collection of fusion algorithms. As shown in Figure 4.1, the unification framework uses the evidences obtained from the input biometric probe data to dynamically select the optimal fusion algorithm. The selected fusion algorithm is then used to compute the fused biometric information. The evidences which serve as input to the unification framework comprise of pertinent and discriminatory attributes such as image quality, features, match scores, and verification prior (precision of true verification score computed from the matcher). Specifically in this chapter, we introduce two novel unification frameworks to combine two match score fusion algorithms: (1) Rule-based unification, (2) Learning-based unification. The first unification framework uses a fixed rule-based strategy to unify fusion algorithms. We further extend the fixed rule-based unification strategy by incorporating an intelligent learning technique based on $2 \nu$-Granular Support Vector Machine for adaptive unification.

In the proposed unification frameworks, we use two fusion algorithms along with the biometric evidences such as image quality, verification prior, and match scores. The first match score fusion algorithm in the proposed unification framework is the evidence theoretic sum rule described in Section 3.2 and the second fusion algorithm is the sequential fusion algorithm described in Section 3.5. These two algorithms are chosen because sum rule is fast and efficient when conflict between classifiers is not large whereas 


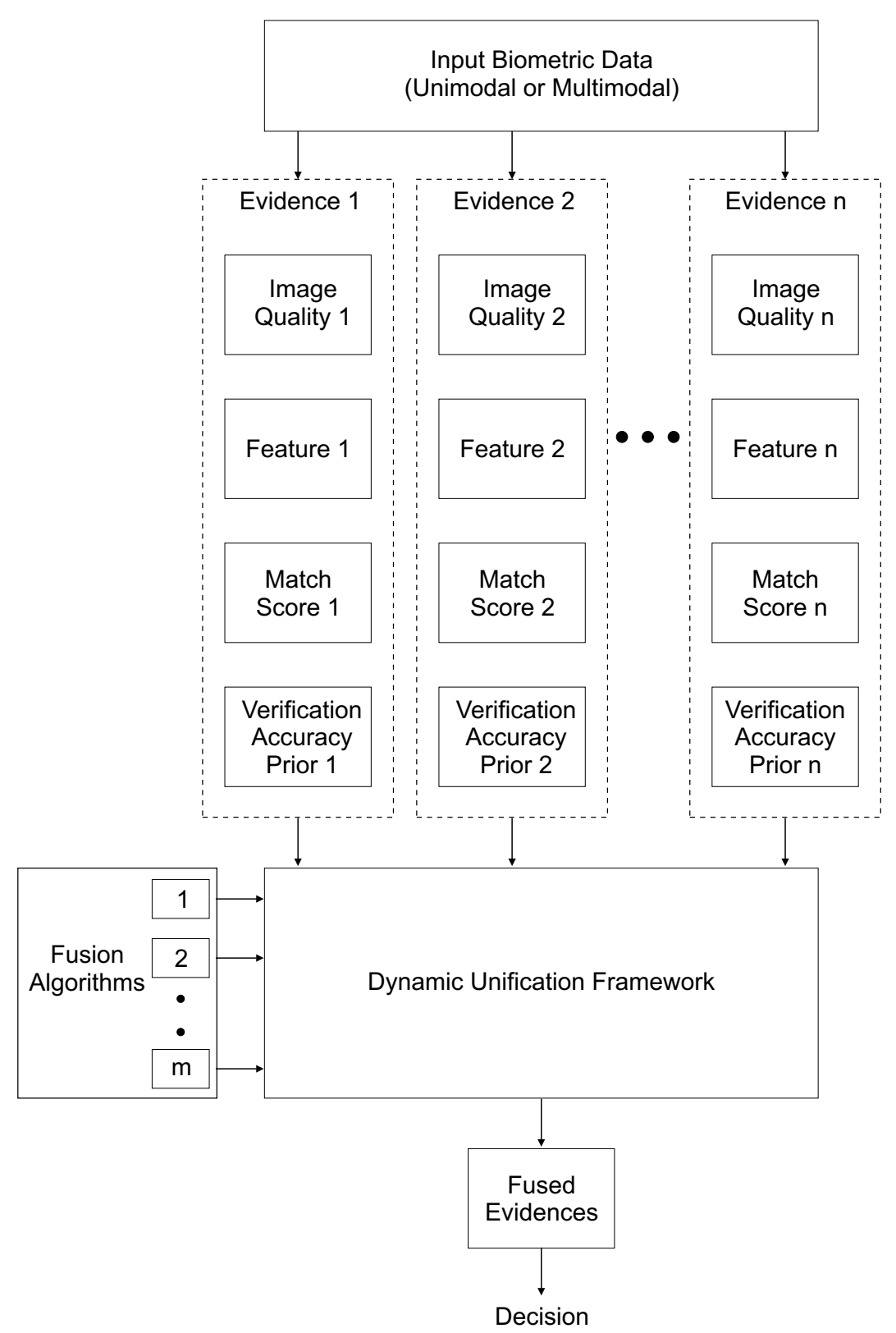

Figure 4.1: General concept of the proposed unification framework. 
sequential fusion algorithm is efficient with large conflicting cases. Depending on the current probe information, the unification framework adaptively selects an appropriate fusion algorithm to fuse level-2 and level-3 match scores. Experimental results show that unification framework reduces the computational time without affecting the verification performance.

\subsection{Framework for Unification of Fusion Algorithms}

Our hypothesis is that the unification of evidence theoretic sum rule and sequential fusion algorithms should provide better verification performance both in terms of accuracy and time. This section describes the proposed unification frameworks in which the two fusion algorithms are unified to improve the verification performance. The rule-based unification is explained first followed by the adaptive unification using $2 \nu$-Support Vector Machine learning.

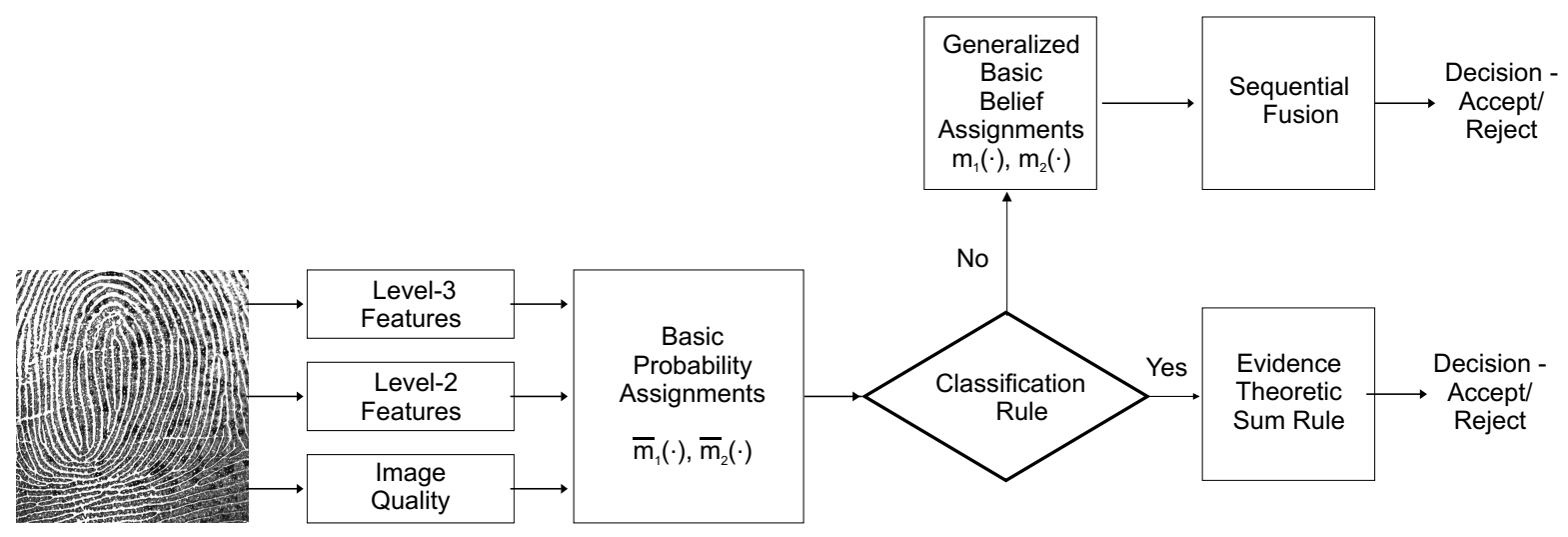

Figure 4.2: Proposed rule-based unification framework. 


\subsubsection{Rule-based Unification}

Figure 4.2 shows the steps involved in the proposed rule-based unification framework. In the rule-based unification, the fixed linear rule shown in Equations 4.1 and 4.2 is used to dynamically select the appropriate fusion algorithm. The procedure for unification is described as follows:

1. The basic probability assignment, $\bar{m}_{i}(j)$ is computed over $\Theta=\left\{\theta_{\text {gen }}, \theta_{\text {imp }}\right\}$ where $i=1,2$ and $j \in \Theta$. The evidence theoretic sum rule is used if the conditions in Equations 4.1 or 4.2 are satisfied.

$$
\begin{aligned}
& \bar{m}_{1}\left(\theta_{\text {gen }}\right)>\bar{m}_{1}\left(\theta_{i m p}\right)+\epsilon_{1} \\
& \bar{m}_{2}\left(\theta_{\text {gen }}\right)>\bar{m}_{2}\left(\theta_{i m p}\right)+\epsilon_{2} \\
& \bar{m}_{1}\left(\theta_{\text {gen }}\right)+\epsilon_{1}<\bar{m}_{1}\left(\theta_{i m p}\right) \\
& \bar{m}_{2}\left(\theta_{\text {gen }}\right)+\epsilon_{2}<\bar{m}_{2}\left(\theta_{i m p}\right)
\end{aligned}
$$

where, $\epsilon_{1}$ and $\epsilon_{2}$ are the error parameters of the two classifiers.

2. If both the above conditions are not satisfied, i.e., when the matchers provide highly conflicting results, then the sequential fusion algorithm is applied for fusion and decision making.

\subsubsection{Adaptive Unification using 2 $\nu$-GSVM Learning}

Unification using the rule-based approach has certain limitations. In the rule-based approach, we first compute basic probability assignment and then the unification conditions (Equations 4.1 and 4.2) are evaluated to decide whether the evidence theoretic sum 
rule or the sequential fusion algorithm should be selected. If the unification condition suggests the use of sequential fusion, both basic probability assignments and generalized basic belief assignments are computed which require additional time. Further, the fixed rule-based unification approach becomes complex if additional fusion rules have to be incorporated in the unification framework and requires some form of optimization. To minimize such complexities, we propose an adaptive unification framework that intelligently learns from the input evidences and selects the appropriate fusion algorithm.

In the proposed adaptive unification framework, we can use any learning strategy to select an appropriate fusion algorithm. As previously mentioned, Support Vector Machine has been widely used in literature for efficient data classification [107]. Chew et

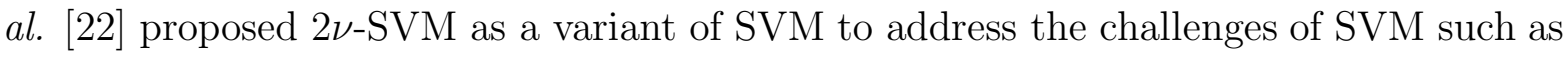
reduction in time complexity and classification with disparate number of training samples per class. The results in the previous chapter show that for multimodal biometric fusion, $2 \nu$-SVM provides better classification with lower time complexity compared to the classical SVM [107]. Further, Tang et al. [100] proposed another variant of SVM by applying granular computing to improve the data classification and time complexity. Granular computing is a knowledge-oriented divide and conquer approach to problem solving in which information is divided into subproblems and these subproblems are solved individually at different granularity levels $[9,10,21,115]$. We use these concepts to incorporate granular computing in $2 \nu$-SVM and formulate the $2 \nu$-Granular SVM [88]. $2 \nu$-GSVM embodies the properties of both granular computing and $2 \nu$-SVM, and utilizes multiple SVMs to learn both local and global properties of the input data at different granularity levels. In [88], it has also been shown that $2 \nu$-GSVM is more adaptive to the data distribution and has lower complexity compared to the classical SVM and 2 $\nu$-SVM. Detailed formulation of $2 \nu$-GSVM is presented in [88]. In the proposed adaptive unification frame- 
work, we thus use $2 \nu$-GSVM to intelligently learn from the input evidences and select the most appropriate fusion algorithm for optimal performance. Figure 4.3 illustrates the computational steps involved in the adaptive unification framework. Image quality and match scores obtained from level-2 and level-3 feature matching are used as input to the $2 \nu$-GSVM classifier. Depending on the output of the $2 \nu$-GSVM classifier, either the sequential fusion or evidence theoretic sum rule is selected for fusion. The adaptive unification is divided into two stages: (1) training and (2) classification.

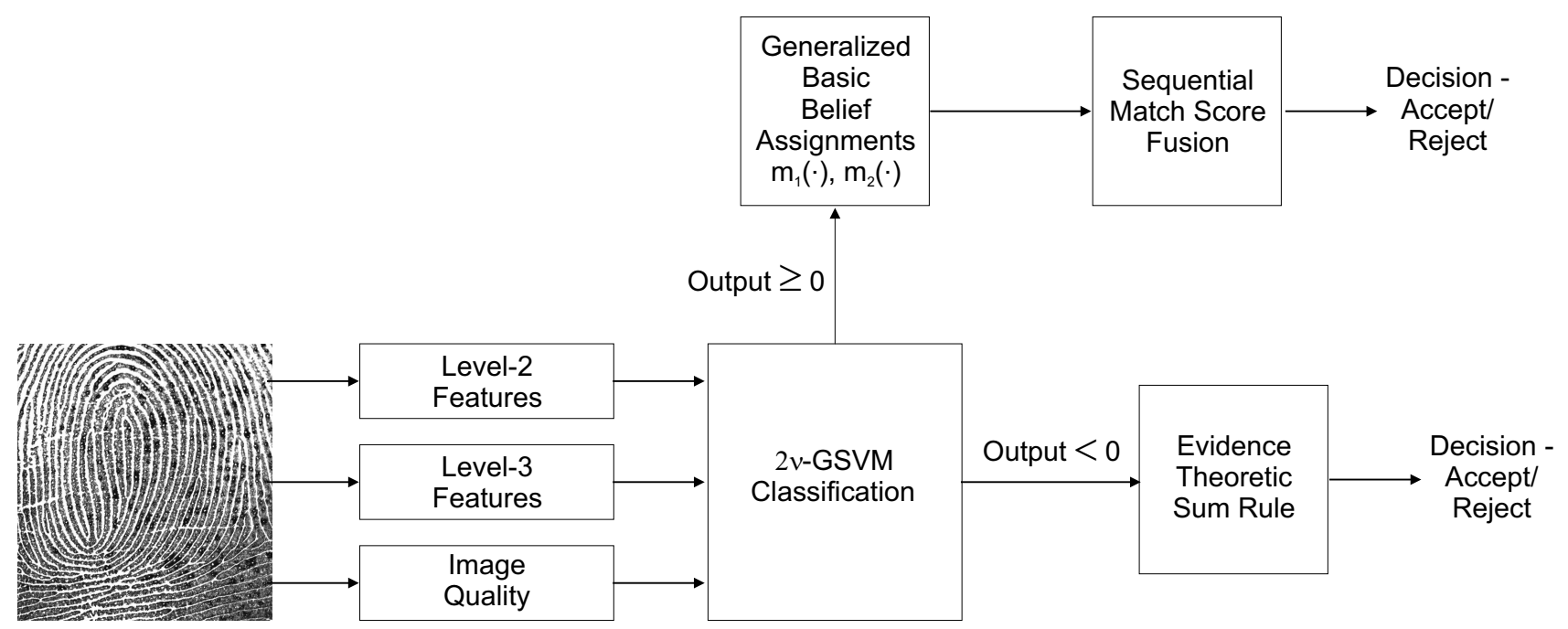

Figure 4.3: Proposed adaptive unification framework using 2 $\nu$-GSVM.

Training 2 $\nu$-GSVM: We train the $2 \nu$-GSVM for unification of the fusion algorithms using a labeled training database. The training algorithm is described as follows:

1. Let the input training data be $\left\{\mathbf{x}_{\mathbf{i}}, y_{i}\right\}$ where $i=1, \ldots, N . N$ is the total number of training samples, $\mathbf{x}_{\mathbf{i}}$ is the $i^{\text {th }}$ data vector that belongs to the binary class $y_{i} . \mathbf{x}_{\mathbf{i}}$ contains the image quality score and two match scores obtained from level-2 and level-3 feature matching. $y_{i} \in(+1,-1)$ is the label such that +1 belongs to the data that should be fused using the sequential match score fusion and -1 belongs 
to the data that should be fused using the evidence theoretic sum rule.

2. $2 \nu$-GSVM is trained using radial basis function kernel such that when the output of $2 \nu$-GSVM $\geq 0$, the sequential fusion is performed and when the output of $2 \nu$-GSVM $<0$, evidence theoretic sum rule fusion is performed.

Classification and Unification: At the probe level, the trained $2 \nu$-GSVM is used to perform classification and unification. The classification algorithm dynamically selects the most appropriate fusion algorithm depending on the quality score and input probe match scores. The steps involved in the classification and unification are described as follows:

1. The image quality score along with the match scores obtained from level-2 and level-3 feature matching are provided as input to the classification algorithm.

2. The trained $2 \nu$-GSVM classifier is used to select the fusion algorithm for the input probe data. The classification algorithm selects either the sequential fusion or evidence theoretic sum rule fusion to fuse the probe match scores and a decision of accept or reject is made.

The adaptive unification framework thus dynamically selects the most appropriate fusion algorithm to improve the verification accuracy and decrease the computational time. The framework can be further generalized to include other fusion schemes with more than two classifiers for other multimodal biometric scenarios.

\subsection{Experimental Results}

The effectiveness of the proposed unification framework is demonstrated experimentally by computing the verification performance of fingerprint biometrics. We also 
evaluate the performance of level-2 and level-3 fingerprint recognition algorithms, evidence theoretic sum rule and sequential match score fusion algorithms, and the proposed unification frameworks with low quality and high quality fingerprint images. The 1000 ppi fingerprint database of 710 classes, the experimental protocol, and the partitioning of train-test database is the same as described in Chapter 3. The algorithms are trained using the training database to compute the mean $\left(\bar{\mu}_{i j}, \mu_{i j}\right)$, standard deviation $\left(\bar{\sigma}_{i j}, \sigma_{i j}\right)$, verification accuracy prior $\left(\bar{V}_{i j}, V_{i j}\right)$, and the error parameters $\left(\epsilon_{1}, \epsilon_{2}\right)$ for the fusion and unification algorithms. The training database is also used to train the $2 \nu$-GSVM classifier for adaptive unification. In this experiment, we compute the verification performance at 0.01\% False Accept Rate.

From Chapter 3, experimental analysis on level-2 matcher, level-3 matcher, evidence theoretic sum rule, and sequential fusion suggest that the performance of evidence theoretic sum rule reduces when the level-2 and level-3 matchers yield highly conflicting results. In such cases, sequential match score fusion algorithm operates on the conflicting region and provides optimal decision using prior information of the unimodal matchers. The sequential match score fusion algorithm thus provides better verification performance. The proposed unification frameworks, both rule based and adaptive, yield a verification accuracy of $98.1 \%$. We found that there are 461 instances when the sequential fusion yields correct classification and the evidence theoretic sum rule provides incorrect classification. However, in both the unification frameworks these cases are correctly classified. Further, there is not a single instance when the sequential fusion produces incorrect results and the evidence theoretic sum rule provides correct results. The advantage of using the unification frameworks over sequential fusion is the computation time. As shown in Table 4.1, the average verification time computed on a 2 GHz Pentium Duo Core processor with 2 GB RAM under MATLAB environment using 
sequential fusion algorithm (including quality assessment, feature extraction, and matching) is approximately 12 seconds. In contrast, the rule-based unification framework takes approximately 11 seconds and the adaptive unification requires around 10 seconds. This shows that the unification frameworks provide higher verification accuracy without increasing the computational time. On comparing both the unification frameworks, we found that the $2 \nu$-GSVM based adaptive unification yields better performance compared to the rule-based unification. Although for this particular case study, the verification accuracies are the same, the computational complexity of the rule-based unification is higher than the adaptive unification. Further, as mentioned in Section 4.1, extending the adaptive unification framework to more than two fusion algorithms is easier and more logical compared to the rule-based unification.

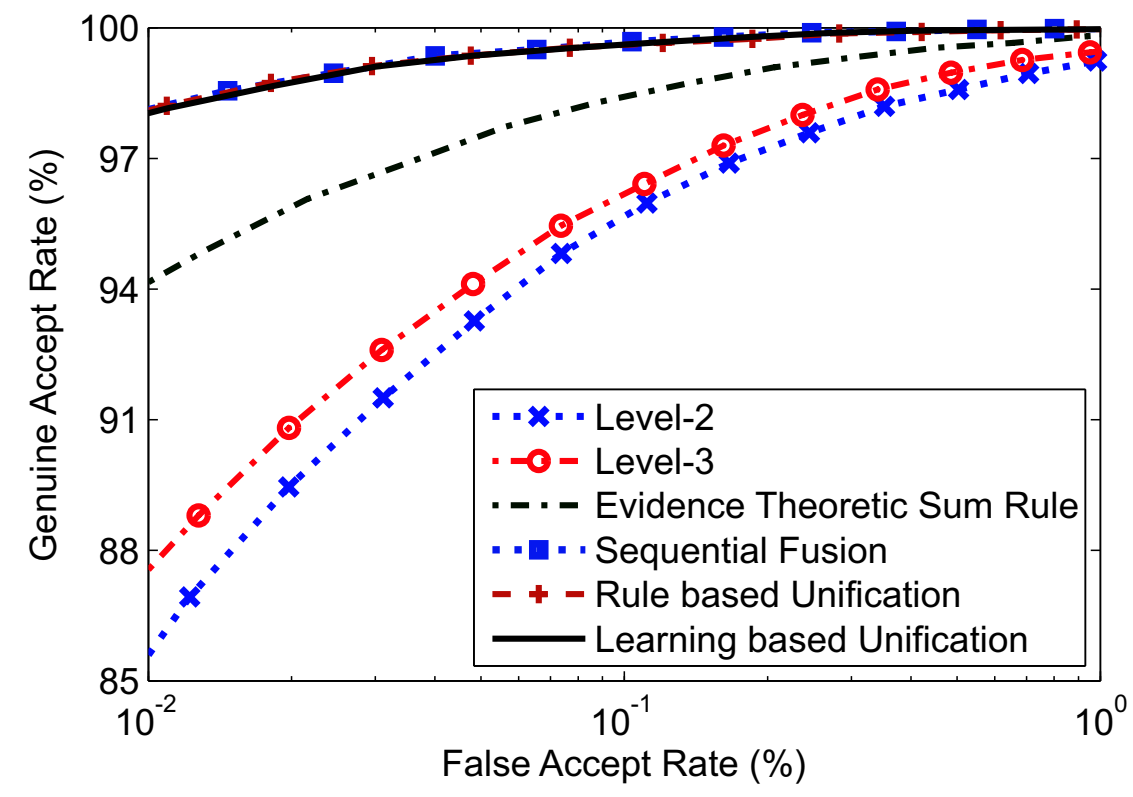

Figure 4.4: ROC plot for evaluating the verification performance of the proposed rulebased and adaptive unification framework using level-2 and level-3 fingerprint features.

To further accentuate the importance of the proposed algorithms, we compute the 
Table 4.1: Comparing the verification performance of the proposed unification framework with match score fusion algorithms using level-2 and level-3 fingerprint features. Verification accuracy is computed at $0.01 \%$ False Accept Rate.

\begin{tabular}{|l|c|c|c|c|}
\hline \multirow{2}{*}{ Algorithms } & \multicolumn{3}{|c|}{ Verification Accuracy (\%) } & Time \\
\cline { 2 - 4 } & $\begin{array}{c}\text { Complete } \\
\text { Database }\end{array}$ & $\begin{array}{c}\text { Low Quality } \\
\text { Images }\end{array}$ & $\begin{array}{c}\text { High Quality } \\
\text { Images }\end{array}$ & (Seconds) \\
\hline $\begin{array}{l}\text { Level-2 } \\
\text { Features } \\
{[48],[55]}\end{array}$ & 85.6 & 80.2 & 88.1 & 03 \\
\hline $\begin{array}{l}\text { Level-3 } \\
\text { Features }\end{array}$ & 87.5 & 81.4 & 90.7 & 05 \\
\hline $\begin{array}{l}\text { Evidence } \\
\text { Theoretic } \\
\text { Sum Rule }\end{array}$ & 94.2 & 92.1 & 96.5 & 09 \\
\hline $\begin{array}{l}\text { Sequential } \\
\text { Fusion }\end{array}$ & 98.1 & 95.8 & 99.2 & 12 \\
\hline $\begin{array}{l}\text { Rule based } \\
\text { Unification } \\
\text { Framework }\end{array}$ & 98.1 & 95.8 & 99.2 & 11 \\
\hline $\begin{array}{l}\text { Adaptive } \\
\text { Unification } \\
\text { Framework }\end{array}$ & 98.1 & 95.8 & 99.2 & 10 \\
\hline
\end{tabular}


verification accuracy with low quality and high quality fingerprint images separately. In this experiment, we study the reason behind lower performance of existing fusion algorithms and how the unification framework manages to yield good performance with conflicting cases. We first use the RDWT quality assessment algorithm described in Chapter 2 to divide the gallery-probe database into two parts: low quality fingerprint database and high quality fingerprint database. The low quality database contains fingerprint images with median quality score between 0 and $0.6\left(0 \leq q_{\text {med }} \leq 0.6\right)$ whereas the high quality database contains images with quality score between 0.6 and $1.0\left(0.6<q_{\text {med }} \leq 1.0\right)$. In the low quality database, there are around 2200 genuine matches and in the high quality database, there are around 3700 genuine matches. Both the low quality and high quality databases are used to compute the verification accuracy of level-2 and level-3 fingerprint recognition algorithms, fusion algorithms, and the proposed rule-based and adaptive unification frameworks at $0.01 \%$ FAR. Table 4.1 shows the verification accuracy for all these algorithms. Analysis of the results are summarized below:

- Relative performance gain of $67.2 \%$ is observed with the sequential fusion compared to the evidence theoretic sum rule. Specifically, with low quality fingerprint images, the proposed sequential fusion algorithm shows significant improvement compared to the evidence theoretic sum rule. Here, it is important to note that with the low quality fingerprint images, level-3 features and level-2 minutia features yield greater number of conflicting results and the performance of evidence theoretic sum rule suffers due to these cases. On the other hand, as mentioned in Chapter 3, sequential fusion yields better performance with conflicting cases by utilizing properties of likelihood ratio, density estimation, belief function theory, and SVM classification. 
- The proposed unification framework yields the best verification performance, both in terms of accuracy and time. With both low and high quality fingerprint images, the verification accuracies of the unification frameworks and sequential fusion are identical. The main advantage of the unification framework over sequential fusion is the reduction in time complexity. For identity verification using the complete database, the adaptive unification framework requires an average of 10 seconds which is two seconds faster than the sequential fusion and only one second slower than the evidence theoretic sum rule. Also, the adaptive unification framework is faster than the rule-based unification framework because the rule based unification framework first computes basic probability assignment to select the fusion algorithm whereas $2 \nu$-GSVM fusion algorithm makes the decision using only the input match scores and quality.

- The experimental results also suggest that in any scenario, the performance of the unification framework will be at least equal to the performance of the weakest fusion rule in the framework. Further, the time required by the unification framework will be less than or equal to the time required by the computationally most expensive fusion algorithm.

- The $t$-test suggests that the unification algorithm is significantly different than the evidence theoretic sum rule but not significantly different than the sequential fusion. However, as shown in Table 4.1, the main advantage of the unification algorithm is reduced computational time without compromising the accuracy. 


\subsection{Summary}

Match score fusion algorithms are optimized for verification accuracy and are not necessarily computationally efficient. Simple fusion algorithms such as evidence theoretic sum rule yield good performance in ideal conditions whereas the sequential fusion algorithm can efficiently combine conflicting match scores at the cost of computational time. In this chapter, we proposed two unification frameworks that dynamically select the most appropriate fusion algorithm depending on the current probe information. The first unification framework is a rule-based approach that selects if the evidence theoretic sum rule or the sequential fusion should be applied depending on the input information. The second unification framework extends the rule-based unification framework by using intelligent $2 \nu$-Granular Support Vector Machine classification to dynamically select the most appropriate fusion algorithm for the best performance. Both the unification frameworks are case based approaches and can be used to improve the verification performance and computational time. To evaluate the proposed algorithms, we used level-2 and level-3 features (single biometrics - multiple matchers). The experimental results suggest that the unification frameworks maintain the verification accuracy and reduce the time complexity. The results also show that fusion of level-2 and level-3 fingerprint features improve the verification performance with both low quality and high quality images. However, $2 \nu$-Granular SVM unification is computationally faster than the rulebased unification. Further, rule-based unification may become complicated with increase in the number of fusion algorithms whereas with SVM unification framework, additional fusion algorithms can be efficiently incorporated by using multiclass classification. 


\section{Chapter 5}

\section{Feature based Watermarking for Secure Multimodal Biometric}

\section{System}

Biometric authentication systems have inherent advantage over traditional personal identification techniques [47]. However, there are many critical issues in designing a biometric system. These issues are broadly characterized by accuracy, computation speed, cost, security, scalability, and real time performance. The security of biometric data is of paramount importance and must be protected from external attacks and tampering [53]. Ratha et al. [72] characterized common attacks in biometric systems as coercive attack, impersonation attack, replay attack, and attacks on feature extractor, template database, matcher, and matching results. Attacks can alter the contents of biometric images or templates and can also degrade the performance of a biometric system. It is therefore required to protect the biometric templates at all times.

Researchers have proposed several algorithms to address the challenges posed for the security of biometric systems. Encryption is one way of addressing this issue and has 
been discussed in $[29,94,106]$. Another way of securing biometric images and templates is by watermarking. Recently, researchers have proposed algorithms based on image watermarking techniques to protect biometric data $[36,53,54,72,109]$. In biometric watermarking, a certain amount of information referred to as watermark, is embedded into the original cover image using a secret key, such that the contents of the cover image are not altered. Some of these methods perform watermarking in the spatial domain $[36,53,54]$ whereas other methods embed the biometric watermark in the frequency domain $[72,109]$. In existing biometric watermarking algorithms, the cover image is either gray scale face image or fingerprint image, and the watermark data is fingerprint minutiae information [53] or face information [54] or iris codes [109].

None of the existing algorithms are designed to embed both level-2 and level-3 features in a host image. The challenge lies in securely embedding these features while the watermark (level-2 and level-3 features) is imperceptible without affecting the biometric content of the host image (face). This process is challenging because the size of level-2 and level-3 features is relatively large compared to other biometric features. Therefore it is difficult to maintain the tradeoff between imperceptibility of watermark and verification performance of face and fingerprint biometrics. In this chapter we propose a novel biometric watermarking algorithm to securely and robustly embed level-2 and level-3 fingerprint features into the color face image of the same individual. Face and fingerprint are chosen for watermarking because of the widespread application of these two biometric modalities. There are several applications such as FBI-AFIS and US-VISIT where face and/or fingerprint are used to authenticate the identity of an individual. The proposed watermarking algorithm first computes the embedding capacity in the face image using edge and corner phase congruency method [57]. Embedding and extraction of fingerprint features is based on Redundant Discrete Wavelet Transformation [25]. The 
performance of the proposed watermarking algorithm is validated using face, fingerprint and multimodal verification algorithms. The experimental results suggest that the proposed watermark embedding and extraction algorithm does not affect the quality of the original face image or the recognition performance of face and fingerprint biometrics. In addition, the proposed algorithm is robust and resilient to common geometric and frequency attacks.

\subsection{Proposed Biometric Watermarking Algorithm}

Usually, image watermarking is performed using Discrete Wavelet Transform because DWT preserves frequency information in a stable form and allows good localization both in spatial and frequency domain $[25,73,97]$. However, one of the major drawbacks of DWT is that the transformation does not provide shift invariance because of the downsampling of its bands. The downsampling causes a major change in the wavelet coefficients of the image even for minor shifts in the input image. In watermarking, we need to know the exact locations of where the watermark information is embedded and the shift variance of DWT may lead to inaccurate extraction of the watermark data and the cover image. To address the issues of DWT based watermarking, researchers have proposed the use of Redundant Discrete Wavelet Transform [16, 34, 39, 40, 42].

Figure 5.1 shows the RDWT decomposition of a face image in four subbands such that the size of each subband is equal to the original image. The redundant space in RDWT provides additional locations for embedding and the watermarking algorithms can be designed such that the exact location of watermark embedding is preserved. In this research, we propose a RDWT biometric watermarking algorithm which not only aims to make the watermark invisible to the human eye and tamper resistant but it also 
ensures that watermark embedding and extraction procedure does not alter the biometric features required for recognition. The proposed watermarking algorithm uses color face image as the cover image. The watermark can be any biometric information such as level-2 and level-3 fingerprint features, iris codes, or voice data. Existing biometric watermarking algorithms use gray scale face images. In this research, we decompose the color face image into three channels which further increases the embedding capacity. Embedding in the red and blue channels makes the watermark imperceptible, while embedding in the green channel makes the watermark visible as noise. The watermarking algorithm involves generating watermark from level-2 and level-3 fingerprint features, computing appropriate locations for embedding the watermark in the face image, embedding fingerprint watermark in these locations, and extracting the watermark for verification.

\subsubsection{Generating Watermark from Level-2 and Level-3 Finger- print Features}

The number of level-2 and level-3 features in a fingerprint image is very large and specifically, pores and ridges contribute towards the large feature size. Due to the tradeoff between verification performance and embedding capacity of a face image, it is not feasible to embed all the fingerprint features in the face image. In order to maintain the verification performance of face biometrics, it is important to reduce the size of the fingerprint watermark data. In this section, an image quality based scheme is presented for generating watermark data from fingerprint images.

First, the fingerprint image is divided into local windows and image quality score of each window is computed using the RDWT based local quality assessment algorithm. Then both level-2 and level-3 features are calculated as described in Section 5.1.2. If 


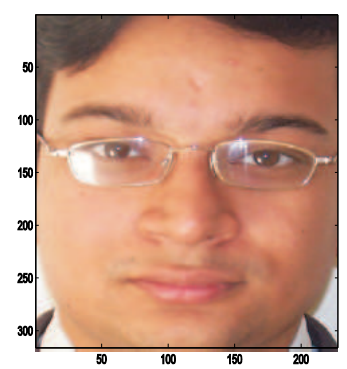

Original image

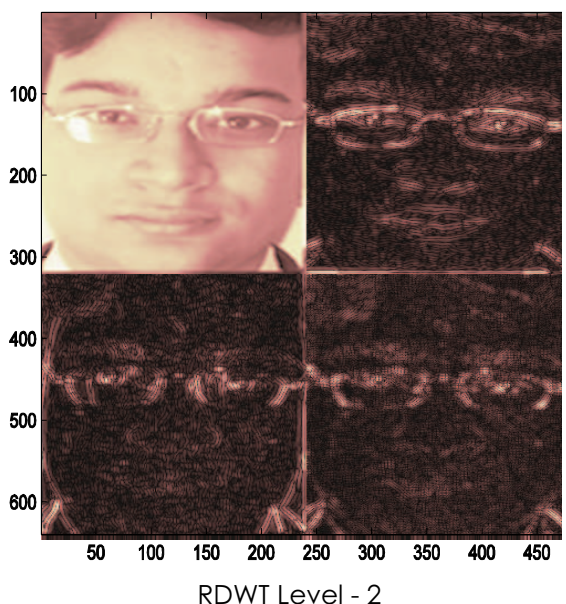

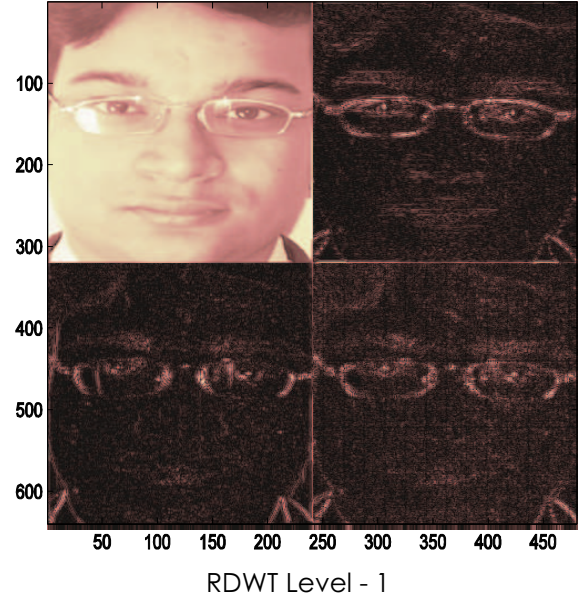

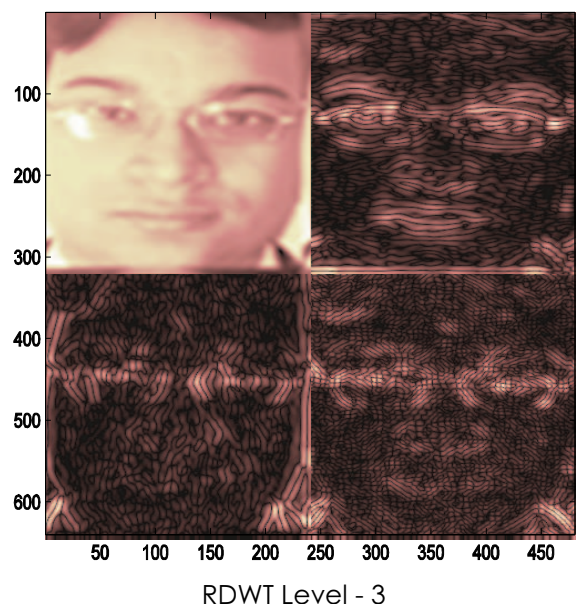

Figure 5.1: RDWT decomposition of a face image. Note that size of all the subbands at every level are same as the original image. 
a window contains a minutia and the quality score is more than a threshold (in our experiments, it is set to 0.5 ), then the window is selected and following features are computed from that particular window:

1. $\left(x_{m}, y_{m}\right)$ coordinates and $\theta_{m}$ of the minutia

2. All the pore coordinates $\left(x_{p}, y_{p}\right)$ in the window

3. Minimum, maximum, and average ridge width using ridge contours

4. Average distance between minutia and pores in the window.

In the same manner, level-2 and level-3 features are computed for all good quality windows and the poor quality windows are discarded. These features are stored in a matrix with one row pertaining to each window. The feature matrix is augmented with dots and incipient ridge features in the last two rows. This feature matrix is used as fingerprint watermark and can be further used for recognition. The size of feature matrix varies from image to image and depends on the image quality. We have performed experiments with slap fingerprint images and at an average, the feature matrix for a slap fingerprint image contains around 1600 elements.

\subsubsection{Computing the Capacity and Locations in Face Image for Watermark Embedding}

Let $f$ denote the color face image of size $(x \times y \times 3)$. The face image is divided into red, green, and blue channels as shown in Figure 5.2. Let $C$ denote the biometric watermark data i.e., fingerprint features of size $(k \times l)$. The watermarking technique should not alter the facial features required for recognition. To, identify the appropriate locations for embedding in a face image, we first compute the edge and corner features in 
the red and the blue channels. RDWT decomposition provides high and low frequency regions which can be used to find the edge and corner features present in the face image. However, as shown in Figure 5.1, facial edge regions cannot be extracted accurately using RDWT decomposition. In our approach, we use phase congruency based edge and corner feature detection algorithm [57]. Since phase congruency is a dimensionless quantity and provides information that is invariant to image contrast, it allows the magnitude of principal moments of phase congruency to be used directly to compute the edges and corners in a face image. Further, phase congruency based edge and corner operator is highly localized [57]. Figure 5.2 shows the phase congruency edge map of the red, green and blue channels of a color face image.

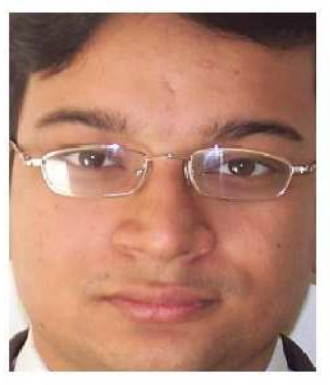

RGB image
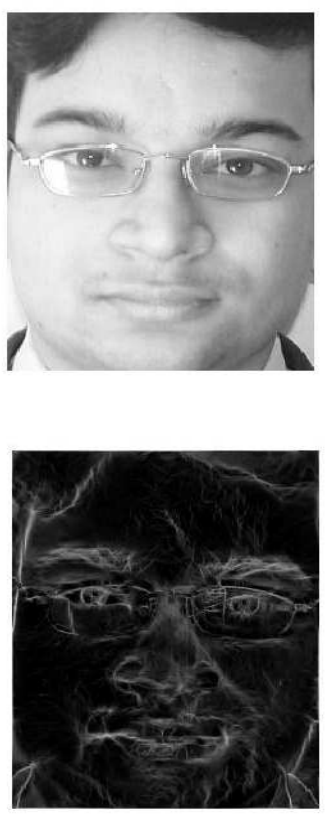

Red channel
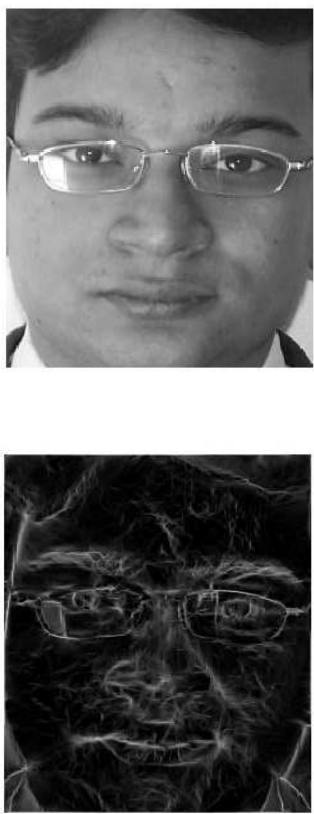

Green channel
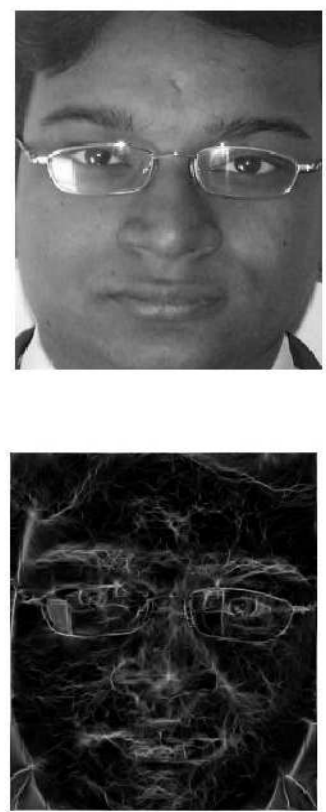

Blue channel

Figure 5.2: Decomposition of color face image into red, green and blue channels, and corresponding phase congruency maps.

Face recognition algorithms use facial features for verification which are usually 
computed along the edge and corner locations in the face image. Embedding biometric watermark in these positions or in their vicinity can affect the performance of face recognition algorithms. Thus regions corresponding to edge and corners computed from the phase congruency method are not used for watermark embedding. The remaining areas of the face image i.e., the low frequency areas are identified as suitable locations for embedding. Red and blue channels of the face image are transformed into $n$ level RDWT with $n \geq 3$. Since the size of each RDWT subband is equal to the size of the input image, three level RDWT decomposition provides adequate capacity to embed the watermark data without affecting the edge and corner features. Only the second and third levels of RDWT are used for embedding because these two levels provide more resilience to geometric and frequency attacks $[61,71]$. We then mark the edge and corner regions in the detailed subbands of the second and third level of red and blue channels, to identify the locations available for embedding.

The size of each subband from the red and blue channel is $(x \times y)$. Let the total size of the regions of interest of the face recognition algorithm in a subband be $(p \times q)$. $(x y-p q)$ denotes the locations available for embedding in a subband, and 12(xy-pq) gives the total locations available for embedding in all subbands of the red and the blue channels. To embed the biometric watermark in the red and blue channels, we first ensure that sufficient locations are available for embedding by applying the condition in Equation 5.1.

$$
12(x y-p q) \geq \sum_{i=1}^{N} m k_{i} l_{i}
$$

where $k \times l$ is the size of the biometric watermark data (fingerprint features), $i=$ $1,2,3, \ldots N$ denotes the number of different biometric templates to be embedded in the biometric cover image and $m=1,2,3 \ldots$ denotes the desired redundancy level of the bio- 
metric watermark data to ensure reliable extraction and processing of multiple copies of the biometric template. This condition shows that we can embed the entire biometric watermark data or different biometric templates or multiple identical biometric templates in the color face image. As $m$ increases, the performance of the watermarking algorithm increases because the algorithm becomes more resilient to different attacks and as $N$ increases, the multimodal verification performance of the algorithm increases. However, for proper reconstruction or extraction of the face image and biometric watermark data, parameter $a$ is introduced in Equation 5.2. The parameter $a$ ensures that the visual quality of the watermarked image does not fall below a certain threshold.

$$
a[12(x y-p q)] \geq \sum_{i=1}^{N} m k_{i} l_{i}
$$

This implies that we have $[6 a(x y-p q)]$ free locations in each of the two channels for embedding the watermark. In this research since we do not use any redundancy of fingerprint features during embedding, $m=1$ and $N=1$. With these values the space available for embedding is much larger than the biometric data to be embedded. $(k \times l) / 2$ locations are randomly selected from the locations available in each of the two channels of the RDWT decomposed face image and these locations are stored as keys $K_{1}$ and $K_{2}$ for the red and blue channels respectively. The keys are used for watermark embedding and watermark extraction.

\subsubsection{Embedding Fingerprint Features in Face Image}

The biometric watermark, i.e. fingerprint features, $C$ of size $k \times l$ is represented as a vector $M(p)$ where $p=1,2, \ldots,(k \times l)$. Color face image $f$ is divided into three channels: $f_{R}$ red channel, $f_{G}$ green channel and $f_{B}$ blue channel. The red and blue channels are 
then transformed into $n$ level RDWT using Daubechies 9/7 mother wavelet [4] to obtain $f_{R}^{r}$ and $f_{B}^{r}$. Fingerprint feature vector $M(p)$ is divided into two parts, $M_{R}$ and $M_{B}$ using Equations 5.3 and 5.4.

$$
\begin{array}{cc}
M_{R}=M(2 z+1) & z=0,1,2,3, \ldots,\lceil p / 2\rceil \\
M_{B}=M(2 z) & z=1,2,3, \ldots,\lfloor p / 2\rfloor
\end{array}
$$

$M_{R}$ is embedded into $f_{R}^{r}$ and $M_{B}$ is embedded into $f_{B}^{r}$ using Equations 5.5 and 5.6 respectively.

$$
\begin{gathered}
f_{R}^{\prime r}(i, j)=f_{R}^{r}(i, j) \stackrel{\text { replace }}{\longrightarrow} \alpha_{1} \times M_{R}\left(z_{1}\right), \quad z_{1}=0,1,2,3, \ldots,\lceil p / 2\rceil \\
f_{B}^{\prime r}(i, j)=f_{B}^{r}(i, j) \stackrel{\text { replace }}{\longrightarrow} \alpha_{2} \times M_{B}\left(z_{2}\right), \quad z_{2}=1,2,3, \ldots,\lfloor p / 2\rfloor
\end{gathered}
$$

Here $(i, j)$ represents the locations in red and blue channels computed using the two keys $K_{1}$ and $K_{2}$ from Section 5.1.1. $\alpha_{1}$ and $\alpha_{2}$ control the strength of the biometric watermark embedded in the red and blue channels respectively. Inverse RDWT is applied on $f_{R}^{\prime r}$ and $f_{B}^{\prime r}$ to obtain the watermarked red and blue channels, $f_{R}^{\prime}$ and $f_{B}^{\prime}$ respectively. Watermarked color face image is then generated by combining the three channels $f_{R}^{\prime}, f_{G}$, and $f_{B}^{\prime}$. Figure 5.3(a) shows the block diagram of the embedding process.

\subsubsection{Extraction of Fingerprint Features from Color Face Image}

Extraction of fingerprint features is the reverse of embedding process. In the extraction process, we assume that the watermarked face image may be subjected to attacks. Let the watermarked face image be $f_{a}$. It is divided into three channels $f_{a R}, f_{a G}$, and 


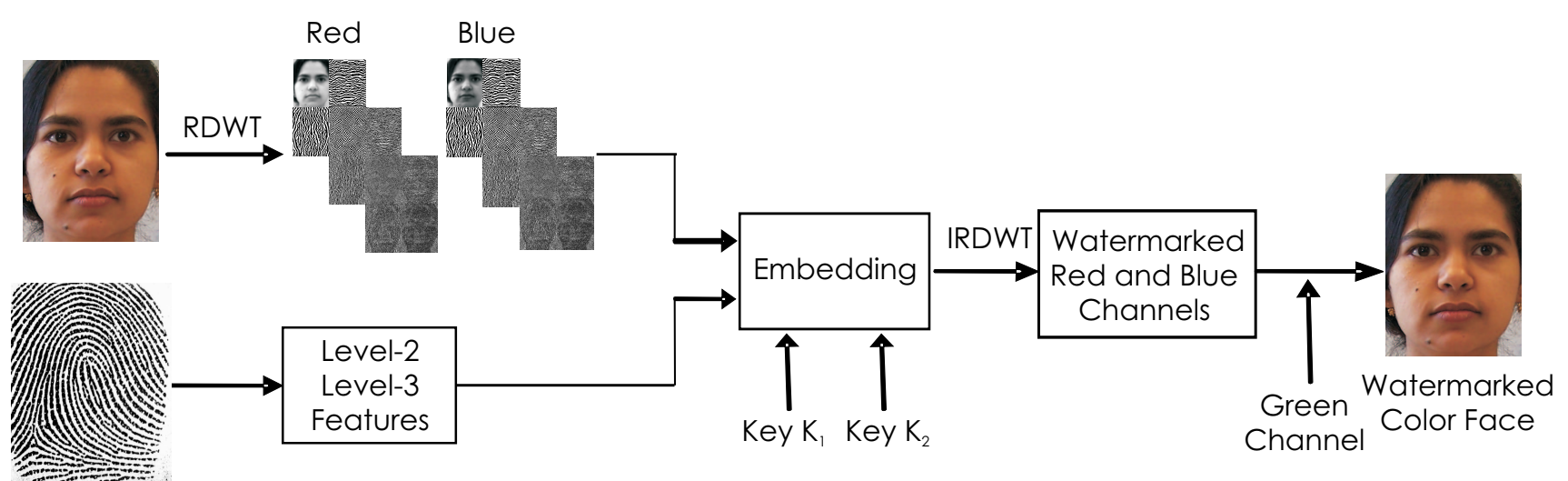

(a)

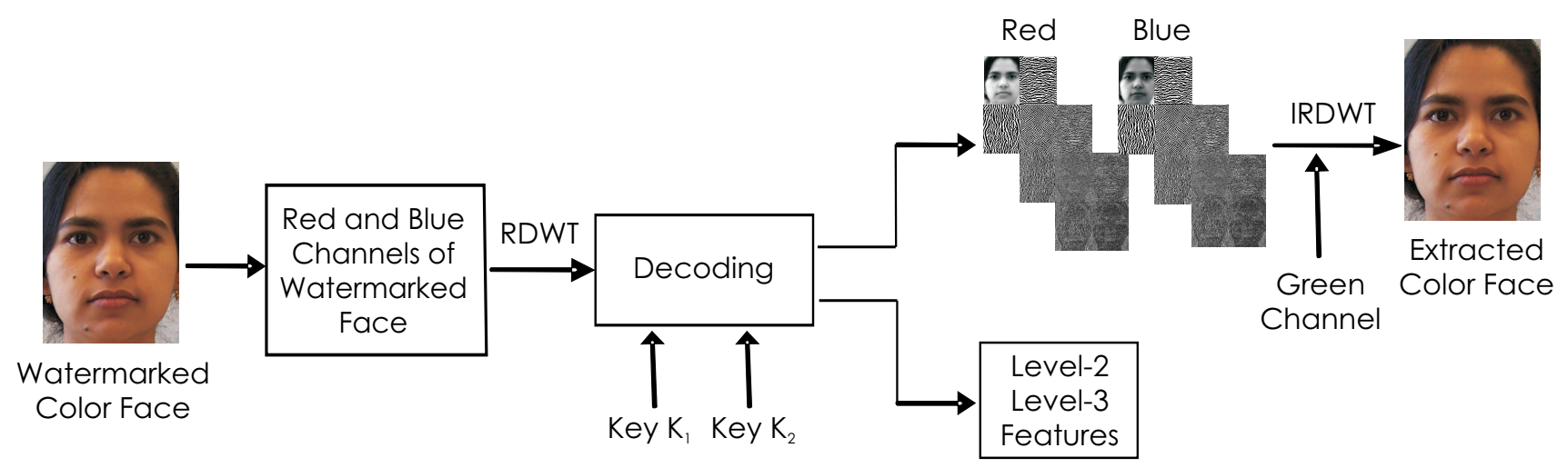

(b)

Figure 5.3: Illustrating the steps involved in the proposed biometric watermarking algorithm (a) embedding process (b) extraction process. 
$f_{a B}$. Applying RDWT using Daubechies 9/7 mother wavelet on the red and blue channels gives $f_{a R}^{\prime}$ and $f_{a B}^{\prime}$. Fingerprint features are extracted from these transformed channels using Equations 5.7 and 5.8.

$$
\begin{array}{ll}
M_{R}^{\prime}\left(z_{1}\right)=\frac{f_{a R}^{\prime}(x, y)}{\alpha_{1}} & z_{1}=0,1, \ldots\lceil p / 2\rceil \\
M_{B}^{\prime}\left(z_{2}\right)=\frac{f_{a B}^{\prime}(x, y)}{\alpha_{2}} & z_{2}=1,2, \ldots\lfloor p / 2\rfloor
\end{array}
$$

where $M_{R}^{\prime}$ and $M_{B}^{\prime}$ are the features extracted from the red and blue channels respectively and $p$ ranges from $1,2, \ldots,(k \times l)$. The keys $K_{1}$ and $K_{2}$ from Section 5.1 .3 give the coordinates for extraction of the fingerprint features. For non-linear reconstruction of the watermark extracted face image, the values in $f_{a R}$ and $f_{a B}$ from where the biometric watermark data is extracted are replaced with zero and IRDWT is applied on the modified image to obtain $f_{a R}^{\prime}$ and $f_{a B}^{\prime}$. Combining $f_{a R}^{\prime}, f_{a G}$, and $f_{a B}^{\prime}$ gives the watermark extracted color face image $f^{\prime}$. The extracted fingerprint features are rearranged in the original vector form using Equation 5.9.

$$
M^{\prime}(p)=M_{R}^{\prime}(1), M_{B}^{\prime}(1), M_{R}^{\prime}(2), M_{B}^{\prime}(2), \quad \ldots \quad M_{R}^{\prime}(i), M_{B}^{\prime}(j)
$$

It is then converted into the feature matrix form for level-2 and level-3 feature based fingerprint verification. Figure 5.3(b) shows the block diagram of the extraction process.

\subsubsection{Algorithmic Complexity}

Let the size of the color face image be $x \times y \times 3$, the size of the fingerprint feature vector be $p$, and the number of levels of RDWT decomposition be $n$. The computational complexity of the embedding process depends on the complexity of the processes shown 
Table 5.1: Computational complexity of the proposed watermarking algorithm.

\begin{tabular}{|l|l|}
\hline Process & Complexity \\
\hline Finding the embedding locations & $O(x \times y)$ \\
\hline RDWT/IRDWT & $O(n \times x \times y)$ \\
\hline Dividing fingerprint features into two parts & $O(p)$ \\
\hline Replacement of values in face image & $O(p)$ \\
\hline Reconstruction of the watermarked color face image & $O(n \times x \times y)$ \\
\hline
\end{tabular}

in Table 5.1.

The complexity of the embedding process is $O(n \times x \times y)$ as $p<<(n \times x \times y)$. The extraction process involves similar steps and hence the complexity of the extraction process is also $O(n \times x \times y)$, where $n<<(x \times y)$.

\subsection{Verifying the Integrity of the Extracted Biomet- ric Data}

To validate the performance of the proposed biometric watermarking algorithm, experiments are performed with the color face image, and level-2 and level-3 features computed from fingerprint image of the same individual. The watermarked face image is stored in the database for recognition. For verification, fingerprint features are extracted from the watermarked face image. The extracted fingerprint features and the face image are matched with the query fingerprint image and face image.

In general watermarking algorithms, the performance is computed based on mea- 
sures such as peak signal-to-noise ratio, mean square error, normalized cross correlation, and histogram similarity. Higher or lower values of these metrics do not ensure higher performance of a biometric system. For a biometric watermarking algorithm, the most important performance metric is the recognition accuracy. The objective of a biometric watermarking algorithm is to provide added security to a biometric system without compromising the quality and features of the biometric cover image and biometric watermark data. To validate the proposed biometric watermarking algorithm, we use verification accuracy of face, fingerprint, and multimodal biometric as the performance metric. The performance of these three biometric modalities is evaluated before embedding and after extraction of the biometric watermark. The verification algorithms and databases used for evaluating the performance of the watermarking algorithm are described in the following subsections.

\subsubsection{Performance Evaluation using Face Verification}

The face region is detected from the image using a triangle based face detection algorithm [84] and the size of the detected face image is $320 \times 240$. The detected face is given as input to the local binary pattern based face verification algorithm [3]. The algorithm computes prominent local binary features from the face and matching is performed using Chi Square measure.

\subsubsection{Performance Evaluation using Fingerprint Verification}

Fingerprint features are extracted using the image quality assessment and feature extraction algorithms described in Chapter 2. The feature matrix is computed using the method described in Section 5.2. The embedded fingerprint features are extracted from 
the face image and then matched with the probe data using the standard bounding box scheme. The composite match score is a distance score normalized in the range of $[0,1]$ using $\min / \max$ normalization.

\subsubsection{Performance Evaluation using Multimodal Biometric}

A single biometric introduces the problem of non-universality and circumvention [78]. To overcome this problem, multiple biometric traits are used for verification. Since the proposed watermarking algorithm uses face and fingerprint, we use a match score level biometric fusion algorithm. The multimodal biometric verification performance is computed using the sequential match score fusion algorithm described in Chapter 3 (Section 3.5).

\subsubsection{Description of Databases}

Experimental validation is performed using a multimodal database of face and fingerprint of 150 individuals. The multimodal database consists of ten samples of each biometric modality for every individual. The size of detected face images is $320 \times 240$. The database is created in two different sessions with a time interval of one week between them; five images of each biometric trait are captured in each of the session. Frontal face images with around $10^{\circ}$ of rotation are captured with varying lighting conditions and facial expressions. Fingerprint database is captured at 1000 dpi so that it can provide both level-2 and level-3 features. The size of the composite level-2 and level-3 feature vector varies according to the number of level-2 minutiae and level-3 features. Since the database is created in different sessions, it contains both inter-class and intra-class variability. Four samples of each individual obtained in the first session are used as training 
data, and the remaining six samples of face and fingerprint are used as testing data to evaluate the verification performance of face, fingerprint, and multimodal biometrics.

\subsection{Computing the Biometric Watermarking Param- eters for Optimal Performance}

In this section, we describe the process for computing the parameters involved in the proposed RDWT biometric watermarking. These parameters are computed to obtain the optimal face, fingerprint and multimodal verification performance. The parameters that affect the performance of RDWT biometric watermarking algorithm are as follows:

- $\alpha_{1}$ and $\alpha_{2}$ control the strength of the watermark fingerprint features during embedding and extraction.

- Parameter $a$ in Equation 5.2 controls the visual quality of the watermarked face image.

- $n$ determines the decomposition level of RDWT.

The parameters of the watermarking algorithm should be chosen so that the extracted face image and the fingerprint features provide maximum verification performance. There are two methods to obtain these parameters. One method is to set these parameters globally so that it is same for all individuals. Another method is to obtain user-specific parameters which are different for every individual depending on his/her facial and fingerprint characteristics. From earlier research in biometrics [51], it is evident that user-specific parameters yield better accuracy than global parameters. Since watermarking is performed during the enrollment stage, it is easy to compute the user-specific 
parameters during enrollment. To compute these parameters, we perform watermarking on face and fingerprint features for different combinations of $\alpha_{1}, \alpha_{2}$, and $a$. The values of $\alpha_{1}$ and $\alpha_{2}$ are varied from 0 to 0.2 with $\alpha_{1}=\alpha_{2}$, and $a$ is varied from 0.05 to 0.4 . The verification performance of multimodal biometrics is computed from the extracted face image and fingerprint features. The values of $\alpha_{1}, \alpha_{2}$ and $a$ which provide the maximum verification performance are chosen. Figure 5.4 shows an example of user-specific parameters associated with different individuals.

The values of the three parameters vary for every individual depending on facial features, skin color, and illumination of the face image. On increasing the value of $\alpha_{1}$ and $\alpha_{2}$, the fingerprint features embedded in the color face image become visible in the form of spurious artifacts and affect the performance of face recognition. On decreasing the value of $\alpha_{1}$ and $\alpha_{2}$, it becomes difficult to reliably extract the fingerprint features, thereby degrading the performance of fingerprint recognition. Parameter $a$ also has similar influence on the biometric watermarking process. Decreasing the value of $a$ decreases the embedding capacity in the face image and increasing the value of $a$ increases the locations available for embedding and allows more fingerprint features to be embedded in the face image. However, if more features are embedded, during extraction of the fingerprint watermark, the quality of the watermark extracted face image decreases due to the non-linear reconstruction, thus decreasing the verification performance of face recognition.

The parameter $n$ denotes the number of levels of RDWT decomposition. Since we use only the second and the third level of decomposition for embedding process, the minimum value of $n=3$. Experiments performed with $n=4,5,6$, and 7 levels did not improve the verification performance but instead increased the time complexity for the watermarking process as shown in Table 5.1. Increasing $n$ however improves robustness 


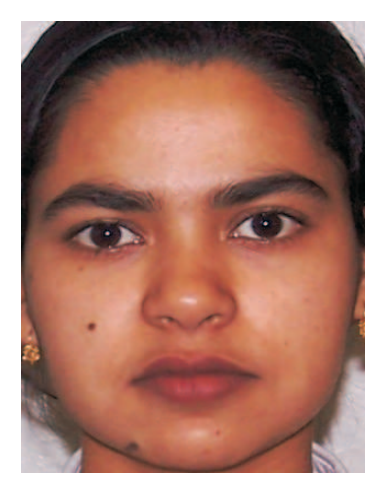

$$
\begin{gathered}
\alpha_{1}=\alpha_{2}=0.009 \\
a=0.14
\end{gathered}
$$

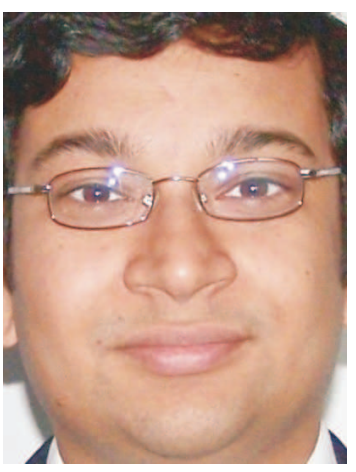

$$
\alpha_{1}=\alpha_{2}=0.014
$$

$\mathrm{a}=0.08$

Figure 5.4: Optimal values of $\alpha_{1}, \alpha_{2}$, and $a$ corresponding to every face image for different individuals.

to attacks due to the redundancy of embedded data.

\subsection{Experimental Evaluation}

The first subsection experimentally substantiates the benefits of RDWT over DWT for the proposed watermarking approach. Section 5.4.2 extends the experimental results of RDWT watermarking by computing the verification performance of face, fingerprint and multimodal biometrics for different attacks on the watermarked face image. This experiment is performed to verify the integrity and robustness of the proposed biometric watermarking algorithm. Section 5.4.3 experimentally evaluates the need for embedding the fingerprint features in low frequency region instead of high frequency regions. 


\subsubsection{Advantage of RDWT over DWT based Biometric Water- marking}

In the proposed algorithm we use RDWT for decomposing the face image. However, existing watermarking algorithms generally use DWT. In this section, we present an experimental evaluation to substantiate the benefits of using RDWT over DWT. The verification performance of face, fingerprint and multibiometric algorithms obtained with RDWT watermarking is compared with the verification performance obtained with DWT watermarking.

For DWT watermarking, we use Daubechies $9 / 7$ mother wavelet and $n=3$, same as in RDWT watermarking. To compute the embedding locations, we downsample the phase congruency edge map to the size of DWT subbands and then perform embedding and extraction. Similar to RDWT watermarking, the parameters $\alpha_{1}, \alpha_{2}$, and $a$ of DWT watermarking are computed separately for every individual.

Figure 5.5 shows the ROC plots of face, fingerprint and multimodal verification for no watermarking, DWT watermarking, and RDWT watermarking. Figure 5.5(a) and (b) show that with DWT watermarking, recognition performance of face, fingerprint, and multimodal biometrics is reduced. However, under similar conditions, Figure 5.5(a) and (c) show that the ROC plots for recognition algorithms before and after RDWT watermarking are almost identical. For both RDWT watermarking and no watermarking, multimodal biometrics algorithm yields an accuracy of $98.8 \%$ whereas the DWT watermarking yields an accuracy of $98.3 \%$. These results validate our choice of selecting RDWT for the proposed biometric watermarking algorithm.

We further analyze the cause for low performance of DWT watermarking with both expansive and non-expansive extension. With expansive extension DWT, we first com- 


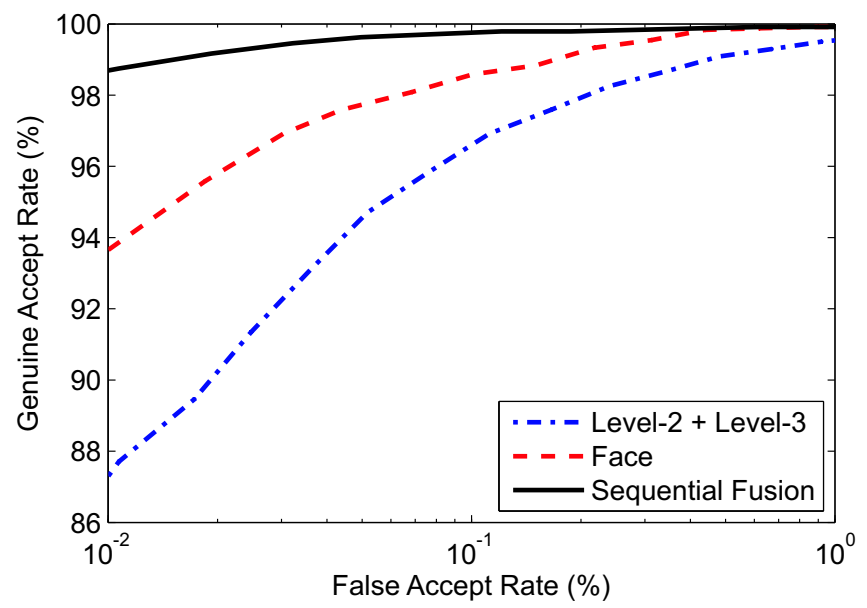

(a)

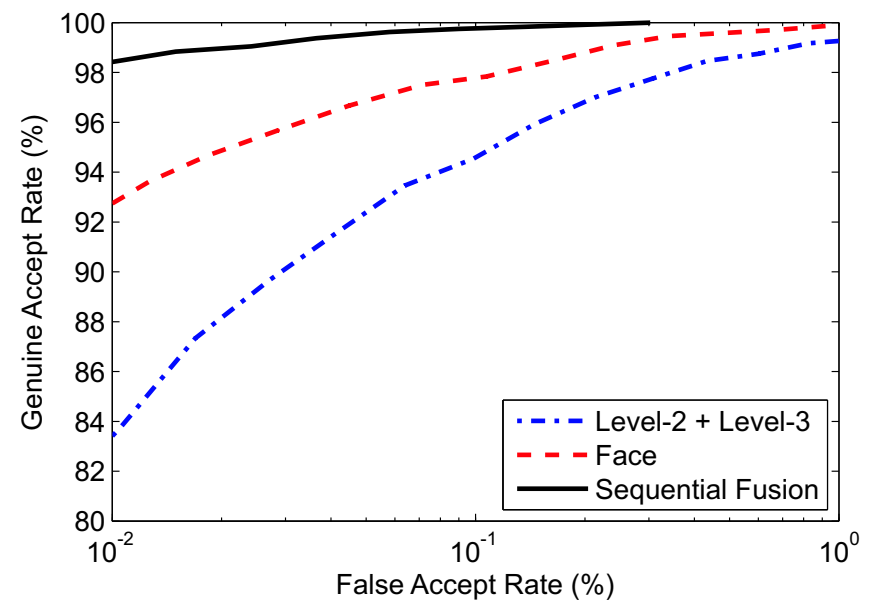

(b)

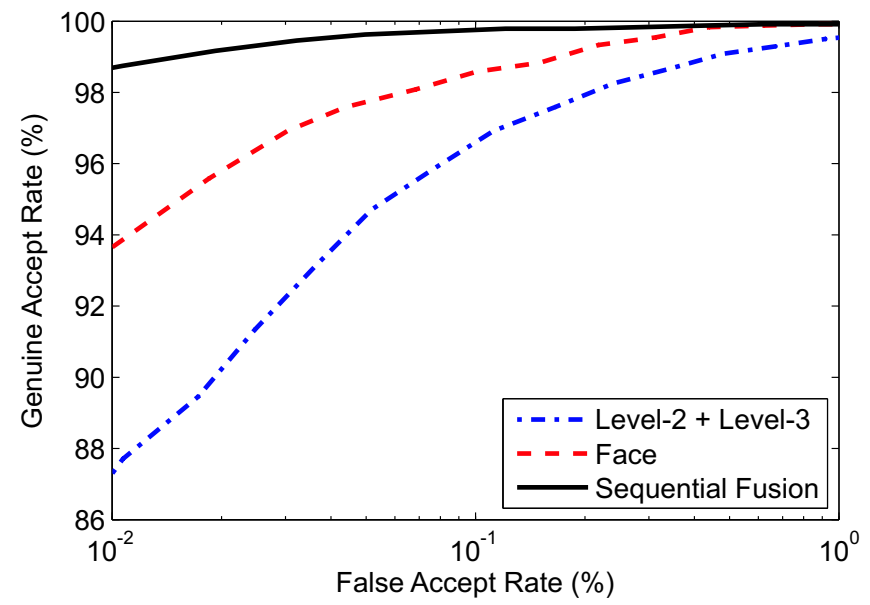

(c)

Figure 5.5: ROC for face, fingerprint, and multimodal biometrics (a) no watermarking (b) DWT watermarking (c) RDWT watermarking. 
$(0,0)$
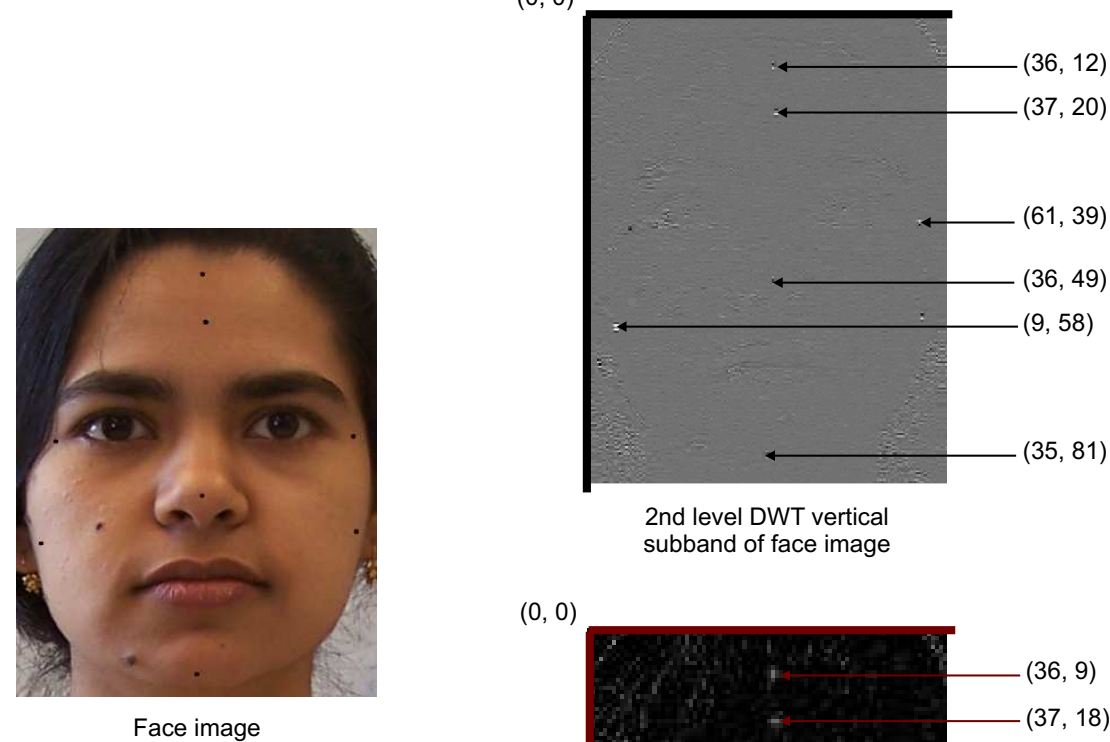

$(0,0)$

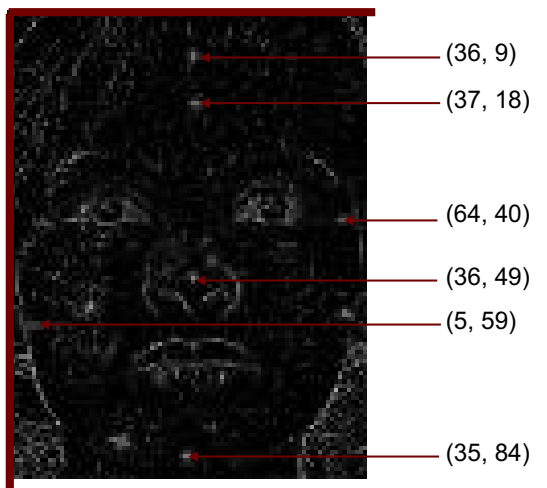

Phase congruency edge map subsampled to the size of 2 nd level DWT vertical subband

Figure 5.6: Face image with second level DWT subband and phase congruency edge map.

Original face image is of size $320 \times 240$, expansive symmetric DWT subband is of size $88 \times 68$, and phase congruency edge map is subsampled to size $88 \times 68$. 
pute the phase congruency edge map and then resize it to the size of DWT subbands. While downsampling, expansive extension DWT adds boundary conditions to the subbands which leads to disparity in the relative coordinates of DWT subband and phase congruency map. As shown in Figure 5.6, the marker position at nose tip $(36,49)$ is same in both the DWT subband and phase congruency edge map; but towards the boundary, the corresponding marker positions in both the images change significantly. This difference causes the inaccurate embedding and extraction of fingerprint features which leads to reduction in the recognition performance. Further, with non-expansive DWT, spurious features due to aliasing at the boundaries cause artifacts in the high frequency subbands, thus compromising the watermarking performance.

\subsubsection{Performance Evaluation of Proposed Biometric Water- marking Algorithm on Attacks}

In the previous subsection, we validated the performance of the proposed RDWT watermarking. For user-specific values of the parameters $\alpha_{1}, \alpha_{2}, a$, and $n=3$, Figure 5.7 shows the original face image, fingerprint feature watermarked face image and the face image after extracting the fingerprint features. Table 5.2 shows that the verification accuracy of face recognition and fingerprint recognition remain the same before embed-

ding and after extraction which further demonstrates that the proposed watermarking does not change the integrity of biometric data embedded in the color face image. The watermark embedding and extraction process may introduce minor variations in some of the fingerprint features or facial characteristics. For example, a minutia coordinate $(201,325)$ when embedded in the face image may change to $(202,323)$ after extraction. However, the biometric recognition algorithms are not sensitive to minor variations at 
these levels and therefore do not affect the verification accuracy. The performance of face recognition does not decrease after RDWT watermarking because the regions of face used by the face verification algorithms are left unchanged during embedding and hence the important facial features remain intact after watermark embedding and extraction.
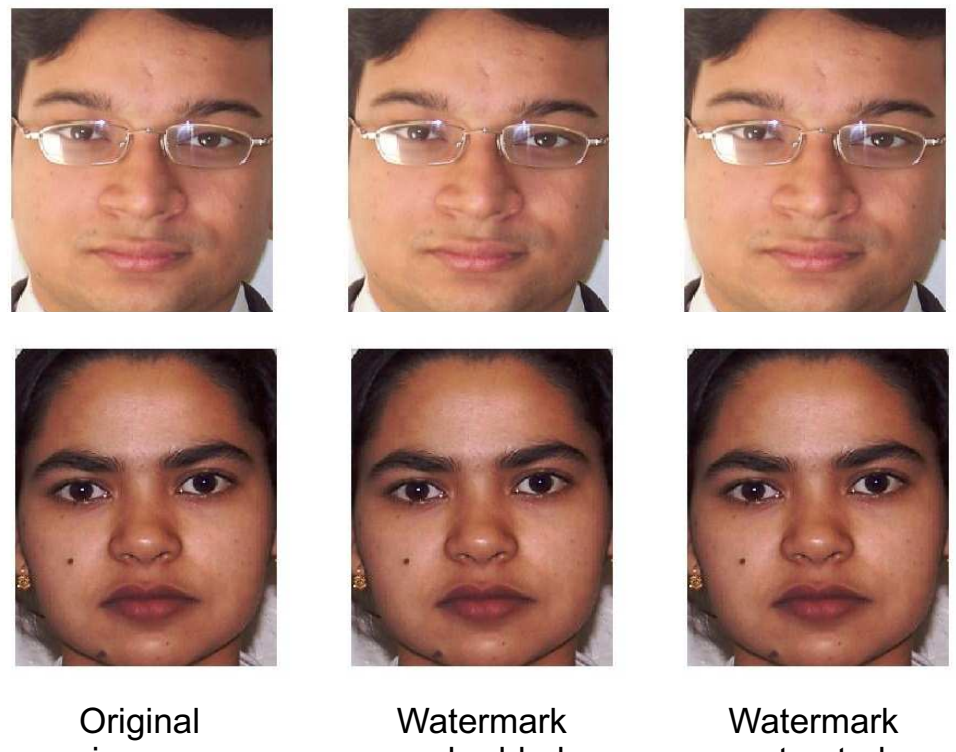

image

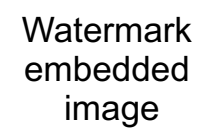
Watermark extracted image

Figure 5.7: Face images showing the effect of watermarking.

Fingerprint features embedded in face image may be vulnerable to low-level signal processing techniques such as compression, low-pass filtering or geometric distortions and may affect the robustness and integrity of the face image and fingerprint features. The watermarking algorithm should be resilient to such attacks. To evaluate the performance of the proposed biometric watermarking algorithm under these conditions, we perform selected frequency and geometry based attacks such as blurring using $3 \times 3$ kernel, filtering with $3 \times 3$ kernel, gamma correction with the gamma constant of 0.5 i.e. the mapping is weighted towards brighter output values, JPEG-2000 compression with 50\% compression rate, rotation by $10^{\circ}$, and scaling with ratio of $1: 1.1$. 

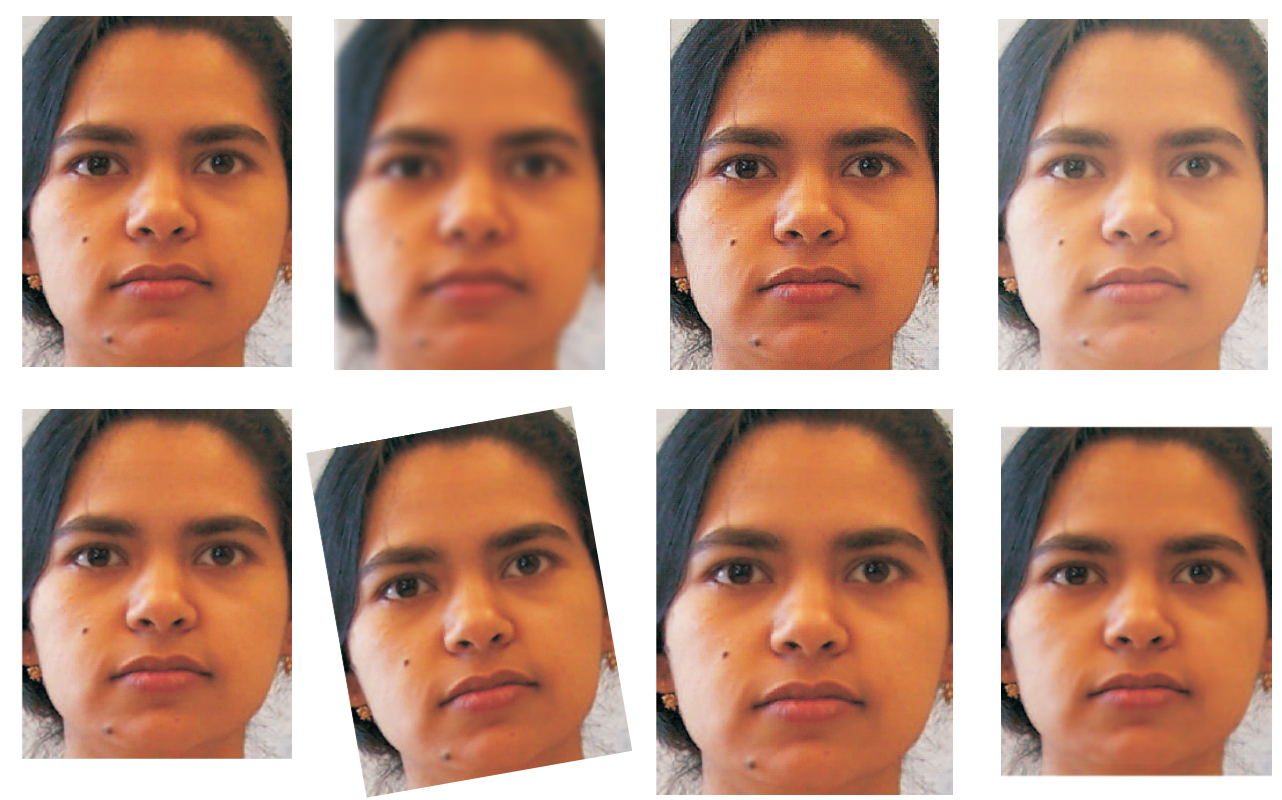

Figure 5.8: Examples of watermarked face image with different attacks (a) original image, (b) blurring, (c) filtering, (d) gamma correction, (e) JPEG compression, (f) rotation, (g) scaling, and (h) feature tampering. 
We next performed attacks on facial feature tampering by randomly altering a single feature in the watermarked face image. In this attack, we manually add one feature to the face image or delete an existing feature. Figure 5.9 shows examples of facial feature tampering. The top row shows images in which moles are removed from the face image and the bottom row shows images in which mustache is added to the face image.
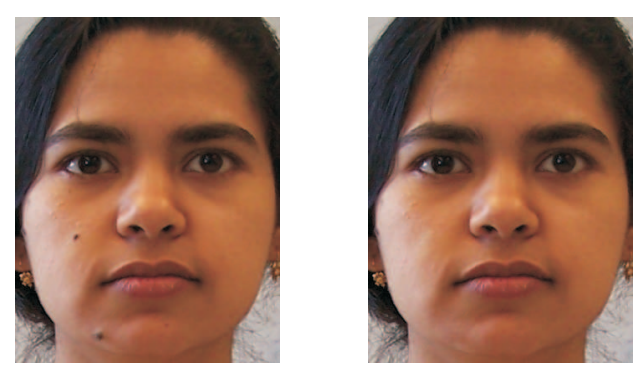

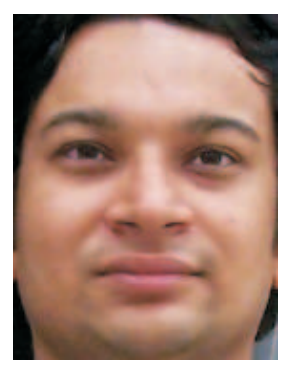

Original Image

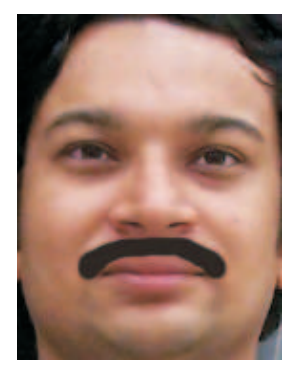

Tampered Image

Figure 5.9: Examples of watermarked face image with facial feature tampering. Top row shows an example of feature removal and bottom row shows an example of feature addition.

These attacks alter the geometric and frequency characteristics of the watermarked color face image. The verification performance of face, fingerprint and multimodal biometrics with and without attacks is summarized in Table 5.2. The blurring and filtering attacks reduce the face verification accuracy by approximately $0.9 \%$, while facial feature alteration reduce the verification performance by $3.6 \%$. We evaluate the performance of facial feature alteration attack without watermarking. Face verification algorithm 
Table 5.2: Verification performance of the proposed biometric watermarking algorithm. Verification accuracy is computed at 0.01\% False Accept Rate.

\begin{tabular}{|c|c|c|c|c|}
\hline \multirow{2}{*}{\multicolumn{2}{|c|}{$\begin{array}{l}\text { Attack scenarios on } \\
\text { watermarked image }\end{array}$}} & \multicolumn{3}{|c|}{ Verification Accuracy (\%) } \\
\hline & & Face & Fingerprint & Multimodal \\
\hline \multicolumn{2}{|c|}{ Without watermarking } & 93.8 & 87.3 & 98.8 \\
\hline \multirow{8}{*}{ With watermarking } & No attack & 93.8 & 87.3 & 98.8 \\
\hline & Blurring $(3 \times 3)$ & 92.9 & 86.5 & 98.2 \\
\hline & Filtering $(3 \times 3)$ & 93.1 & 86.9 & 98.4 \\
\hline & Gamma (0.5) & 93.8 & 87.3 & 98.8 \\
\hline & JPEG-2000 (50\%) & 93.8 & 87.2 & 98.8 \\
\hline & Rotation $\left(10^{0}\right)$ & 93.8 & 86.4 & 98.0 \\
\hline & Scaling $(1: 1.1)$ & 92.5 & 86.7 & 98.3 \\
\hline & Facial Feature Tampering & 90.2 & 84.8 & 97.5 \\
\hline
\end{tabular}


provides an accuracy of $90.2 \%$ which is equal to the accuracy obtained when the watermarked face image is subjected to feature tampering attack. Analysis of these results confirm that the decrease in accuracy is due to feature tampering and not due to watermarking. These results thus show that the overall performance of the proposed biometric watermarking algorithm is resilient to attacks.

We also study the integrity of embedded fingerprint features when subjected to various attacks. Table 5.2 shows that blurring, filtering and rotation attacks on the biometric watermarked face image cause minimal decrease in the accuracy compared to the original accuracy of $87.3 \%$ when there are no attacks. It is interesting to note that when the original fingerprint image is directly subjected to a filtering attack using the same kernel size of $3 \times 3$, the performance of the fingerprint verification reduced to $86.9 \%$. This shows that the fingerprint features when embedded in the face image provide additional level of protection from attacks. For feature alteration attack, the performance of fingerprint recognition decreases by $2.5 \%$. This is because some of the facial feature alteration attacks such as adding beard and mustache change the characteristics of nonfeature or low frequency regions where fingerprint features are embedded. However, the deletion of features do not cause any error because the fingerprint features are embedded away from the facial features. The results summarized in Table 5.2 show that biometric watermarking provides an additional layer of protection to the biometric fingerprint features, enhances security, and is resilient to attacks. For applications where higher biometric accuracy and more robustness to attacks is desired, Table 5.2 further shows that the combination of multiple biometrics with sequential match score fusion yields a multimodal accuracy of $98.8 \%$ with around $1 \%$ degradation in performance when the watermarked face image is subjected to various attacks. 


\subsubsection{Effect of Embedding Watermark Data in High and Low Frequency Regions}

The proposed watermarking algorithm embeds the fingerprint features as watermark in low frequency regions or non-feature regions of the face image. Traditional watermarking algorithms embed watermark in high frequency regions so that the watermark with higher energy can also be embedded without making it perceptible [16]. We compare the verification accuracy of face images when fingerprint features are embedded in low frequency regions and when fingerprint features are embedded in high frequency regions. We study the performance using five face verification algorithms namely, Principal Component Analysis [104], Fisher Linear Discriminant Analysis [12], Geometric features [24], Local Feature Analysis [7], and Local Binary Pattern [3]. PCA and FLDA were chosen because they represent appearance based algorithms, geometric features and LFA represent feature based algorithms, whereas LBP represents texture based algorithms.

Table 5.3 shows that for all five face verification algorithms, embedding in low frequency regions yields better performance than embedding in high frequency regions. Using the verification accuracy of non-watermarked face images as reference, the verification accuracy of high frequency embedding decreased by $1.3 \%$ to $4.2 \%$. On the other hand, embedding in low frequency regions resulted in a smaller decrease in the range of $0.0 \%$ to $1.5 \%$ for the five verification algorithms.

\subsection{Summary}

With the increased use of biometric systems, the possibility of attacks on the biometric images and templates also increases. In this chapter, we proposed a feature based watermarking algorithm to protect the biometric templates in a multimodal biometric 
Table 5.3: Performance comparison of embedding level-2 and level-3 fingerprint features in high frequency regions and in low frequency regions. Face verification accuracy is computed at $0.01 \%$ FAR.

\begin{tabular}{|l|c|c|c|}
\hline \multirow{2}{*}{ Face Verification } & \multicolumn{3}{|c|}{ Verification Accuracy (\%) } \\
\cline { 2 - 4 } & $\begin{array}{c}\text { Without } \\
\text { Watermarking }\end{array}$ & $\begin{array}{c}\text { Watermarking in High } \\
\text { Frequency Regions }\end{array}$ & $\begin{array}{c}\text { Watermarking in Low } \\
\text { Frequency Regions }\end{array}$ \\
\hline PCA [104] & 63.2 & 61.9 & 62.1 \\
\hline FLDA [12] & 67.5 & 64.3 & 66.0 \\
\hline Geometric Features [24] & 88.7 & 87.1 & 88.7 \\
\hline LFA [7] & 90.1 & 88.4 & 90.1 \\
\hline LBP [3] & 93.8 & 89.6 & 93.8 \\
\hline
\end{tabular}


system. Using redundant discrete wavelet transform, level-2 and level-3 features are embedded into the color face image while preserving the facial features. The robustness of the watermarking algorithm is evaluated by comparing the recognition accuracies of face, fingerprint, and multimodal biometric algorithms. Experimental results show that the proposed biometric watermarking algorithm is resilient to different signal processing attacks with decrease of $0-1.3 \%$ in multimodal biometric verification accuracy. Further, evaluation using different appearance, feature, and texture based face recognition algorithms demonstrate that the proposed watermarking algorithm does not alter the biometric information required for recognition. In conclusion, the proposed algorithm has following major properties: (1) perceptual transparency, (2) watermark detection does not require the original image, (3) detector is able to locate watermark even after attacks, and (4) verification performance is not compromised. 


\section{Chapter 6}

\section{Conclusions and Future Work}

\subsection{Conclusions}

Fingerprint images, captured using a high resolution optical scanner, provide abundant amount of information useful for verifying the identity of an individual. However, the main challenge lies in reliably extracting and utilizing these features in such a manner that it can address the inter-class and intra-class variability of biometric data. Further, security of fingerprint templates is also an important challenge as any attack or tampering can severely impact the biometric system. Therefore, the objective of this dissertation is to design a reliable, fast and secure fingerprint recognition system using extended feature set.

In this research, we first designed a curve evolution approach based feature extraction algorithm to extract pores, ridge contours, dots and incipient ridges. RDWT based local quality assessment algorithm is designed and quality scores are incorporated in the likelihood ratio based matching scheme. Experiments with 1000 ppi rolled and slap fingerprint images show that level-3 features are highly discriminating and the proposed quality induced fingerprint matching algorithm yields better accuracy compared to existing level-2 and level-3 feature based recognition algorithms. 
Experimental analysis suggests that fusion of match scores obtained from level-2 and level-3 matchers can enhance the verification accuracy. Therefore, we next proposed five different fusion algorithms to combine multiclassifier fingerprint information. The fusion algorithms are: (1) Delaunay triangulation based fusion algorithm, (2) evidence theoretic sum rule, (3) $2 \nu$-support vector machine fusion algorithm, (4) proportional conflict redistribution rule based fusion algorithm, and (5) sequential match score fusion algorithm. These algorithms incorporate image quality score with level-2 and level-3 features or match scores to improve the performance. Among the five algorithms, evidence theoretic sum rule is the fastest whereas sequential fusion algorithm, that incorporates the density-based fusion scheme into a belief model and use likelihood ratio test-statistic with a support vector machine, is the best for classification and yields the best accuracy.

To efficiently address both accuracy and time complexity of multiclassifier fingerprint recognition, we next proposed an unification framework that reconciles evidence theoretic sum rule with sequential fusion algorithm. Specifically, we designed the rule based unification framework and adaptive learning based unification framework to combine match score fusion algorithms. The first unification framework uses a fixed rulebased strategy to unify fusion algorithms whereas the second framework incorporates an intelligent learning technique for adaptive unification. The unification frameworks are optimized to provide optimal performance both in terms of accuracy and time.

Finally, we designed a novel RDWT based watermarking algorithm to secure face and fingerprint bimodal biometric system from external attacks and tampering. In the proposed watermarking algorithm, color face image is used as the host image and level2 and level-3 fingerprint features extracted from the fingerprint image are used as the watermark. The proposed algorithm provides additional layer of security against different tampering attacks without affecting the quality of the original face image or the 
multimodal verification performance.

\subsection{Future Research Directions}

Research in level-3 fingerprint recognition is continuing to emerge and there are several topics that can be further explored to improve the performance. A selected list of potential topics are summarized below:

- Since automatic level-3 feature extraction and matching is a relatively new technique, a scientific study is required to analyze the permanence, universality, and sensor interoperability. Undertaking such research requires a comprehensive large scale fingerprint database that not only contains temporal variations but also variations due to geography, gender and race.

- The curve evolution based level-3 feature extraction algorithm can be extended such that during tracing, level-2 minutia features can also be computed. This extension may reduce the computational complexity of level-2 and level-3 feature extraction. Furthermore, additional research is required to study the applicability of local quality scores in fingerprint feature extraction.

- The potential of using the proposed match score fusion algorithms, especially the sequential fusion algorithm, can be evaluated for other multimodal cases. Similarly, the proposed unification framework can also be used for other multimodal scenarios such as match score fusion of multiple biometric modalities (face and fingerprint, or face and iris).

- Additional research is required to extend the proposed unification frameworks to include multi-matchers, multilevel fusion algorithms and multiple biometric modal- 
ities. In such cases, the use of multiclass SVM classification can be explored to dynamically select the best fusion algorithm. 


\section{Appendix}

\section{Appendix A}

\section{Dissemination of Research Results}

1. M. Vatsa, R. Singh, A. Noore, and M. Houck, Quality-augmented fusion of level2 and level-3 fingerprint information using DSm theory, International Journal of Approximate Reasoning, 2009 (In Press).

2. M. Vatsa, R. Singh, and A. Noore, Unification of evidence theoretic fusion algorithms: a case study in level-2 and level-3 fingerprint features, IEEE Transactions on Systems, Man, and Cybernetics-A, Vol. 39, No. 1, 2009.

3. M. Vatsa, R. Singh, and A. Noore, Feature based RDWT watermarking for multimodal biometric system, Image and Vision Computing - Special Issue on Multimodal Biometrics, Vol. 27, No. 3, pp. 293-304, 2009.

4. R. Singh, M. Vatsa, and A. Noore, Integrated multilevel image fusion and match score fusion of visible and infrared face images for robust face recognition, Pattern Recognition - Special Issue on Multimodal Biometrics, Vol. 41, No. 3, pp. 880-893, 2008.

5. M. Vatsa, R. Singh, and A. Noore, Belief model fusion in multimodal biometrics, Advances and Applications of DSmT for Information Fusion - Vol. III, Edited by F. Smarandache and J. Dezert, American Research Press, 2008 (Invited Chapter). 
6. M. Vatsa, R. Singh, A. Noore, and S.K. Singh, Belief function theory based biometric match score fusion: case studies in multi-instance and multi-unit iris verification, In Proceedings of International Conference on Advances in Pattern Recognition, 2009 (In Press).

7. M. Vatsa, R. Singh, A. Noore, and S.K. Singh, Quality induced fingerprint identification using extended feature set, In Proceedings of IEEE Conference on Biometrics: Theory, Applications and Systems, pp. 1-6, 2008.

8. M. Vatsa, R. Singh, A. Ross, and A. Noore, Likelihood ratio in a SVM framework: fusing linear and non-linear face classifiers, In Proceedings of IEEE Computer Society Workshop on Biometrics at the Computer Vision and Pattern Recognition Conference, pp. 1-6, 2008.

9. M. Vatsa, R. Singh, and A. Noore, SVM Fusion of multimodal biometric match scores with image quality metric, International Journal of Neural Systems, Vol. 17, No. 5, pp. 880-893, 2007.

10. A. Noore, R. Singh, M. Vatsa, and M.M. Houck, Enhancing security of fingerprints through contextual biometric watermarking, Forensic Science International, Vol. 169, No. 2-3, pp. 188-194, 2007.

11. A. Noore, R. Singh, and M. Vatsa, Robust memory efficient data level information fusion of multimodal biometric images, Information Fusion, Vol. 8, Issue 4, pp. $337-346,2007$.

12. A. Noore, M. Vatsa, R. Singh, M. Houck, and K. Morris, Multiple watermarking enhances security of fingerprint images, Journal of Document and Identity, Vol. 22, pp. 3-6, 2007. 
13. M. Vatsa, R. Singh, and A. Noore, Unification of evidence theoretic fusion algorithms: a case study in level-2 and level-3 fingerprint features, In Proceedings of IEEE Conference on Biometrics: Theory, Applications and Systems, pp. 1-6, 2007 Received the Honeywell Best Student Paper Award.

14. R. Singh, M. Vatsa, A. Noore, and S.K. Singh, DS Theory Classifier Fusion with Update Rule to Minimize Training Time, IEICE Electronics Express, Vol. 3, No. 20, pp. 429-435, 2006.

15. M. Vatsa, R. Singh, A. Noore, M. Houck, and K. Morris, Robust Biometric Image Watermarking for Fingerprint and Face Template Protection, IEICE Electronics Express, Vol. 3, No. 2, pp. 23-28, 2006.

16. R. Singh, M. Vatsa, and A. Noore, Intelligent biometric information fusion using support vector machine, Soft Computing in Image Processing: Recent Advances by Springer edited by M. Nachtegael, D. Van der Weken, E.E. Kerre, and W. Philips, Chapter 12, pp. 327-350, 2006.

17. R. Singh, M. Vatsa, A. Noore, and S. K. Singh, Dempster shafer theory based classifier fusion for improved fingerprint verification performance, In Proceedings of Indian Conference on Computer Vision, Graphics and Image Processing, LNCS Vol. 4338, pp. 941-949, 2006.

18. M. Vatsa, R. Singh, and A. Noore, Enhancing accuracy and security of multibiometric template using RWDT watermarking, In Proceedings of Summer School on Biometric Authentication: Multimodality and System Integration, 2005, Received the Best Paper Award.

19. M. Vatsa, R. Singh, and A. Noore, Improving biometric recognition accuracy and 
robustness using dwt and SVM watermarking, IEICE Electronics Express, Vol. 2, No. 12, pp. 362-367, 2005.

20. M. Vatsa, R. Singh, P. Mitra, and A. Noore, Digital watermarking based secure multimodal biometric system, In Proceedings of IEEE International Conference on Systems, Man and Cybernetics, Vol. 3, pp. 2983-2987, 2004.

21. M. Vatsa, R. Singh, P. Mitra, and A. Noore, Comparing robustness of watermarking algorithms on biometrics data, In Proceedings of Workshop on Biometric Challenges from Theory to Practice (ICPR Workshop), 2004. 


\section{Bibliography}

[1] J.F. Aguilar, J.O. Garcia, J.G. Rodriguez, and J. Bigün, "Kernel-based multimodal biometric verification using quality signals," In Proceedings of SPIE Biometric Technology for Human Identification, Vol. 5404, pp. 544-554, 2004.

[2] J.F. Aguilar, J.O. Garcia, J.G. Rodriguez, and J. Bigün, "Discriminative multimodal biometric authentication based on quality measures," Pattern Recognition, Vol. 38, No. 5, pp. 777-779, 2005.

[3] T. Ahonen, A. Hadid, and M. Pietikinen, "Face description with local binary patterns: application to face recognition," IEEE Transactions on Pattern Analysis and Machine Intelligence, Vol. 28, No. 12, 2006. 2037-2041.

[4] M. Antonini, M. Barlaud, P. Mathieu, I. Daubechies, "Image coding using wavelet transform," IEEE Transactions on Image Processing, Vol. 1, No. 2, pp. 205-220, 1992.

[5] M. Arif, T. Brouard, and N. Vincent, "A fusion methodology based on DempsterShafer evidence theory for two biometric applications," In Proceedings of 18th International Conference on Pattern Recognition, Vol. 4, 2006. 590-593.

[6] D.R. Ashbaugh, "Quantitative-qualitative friction ridge analysis: an introduction to basic and advanced ridgeology," CRC Press, 1999.

[7] J.J. Atick and P.S. Penev, "Local feature analysis: a general statistical theory for object representation," Network: Computation in Neural System, Vol. 7, No. 3, pp. 477-500, 1996.

[8] P. Bao, L. Zhang, and X. Wu, "Canny edge detection enhancement by scale multiplication," IEEE Transactions on Pattern Analysis and Machine Intelligence, Vol. 27, No. 9, pp. 1485-1490, 2005. 
[9] A. Bargiela and W. Pedrycz, "Granular computing: an introduction," Kluwer International Series in Engineering and Computer Science, 2002.

[10] A. Bargiela and W. Pedrycz, "The roots of granular computing," In Proceedings of IEEE International Conference on Granular Computing, pp. 806-809, 2006.

[11] G. Bebis, T. Deaconu, and M. Georgiopoulos, "Fingerprint identification using delaunay triangulation," In Proceedings of IEEE International Conference on Intelligence, Information and Systems, pp. 452-459, 1999.

[12] P.N. Belhumeur, J. P. Hespanha, and D. J. Kriegman, "Eigenfaces vs. fisherfaces: recognition using class specific linear projection," IEEE Transactions on Pattern Analysis and Machine Intelligence, Vol. 19, No. 7, pp. 711-720, 1997.

[13] E.S. Bigün, J. Bigün, B. Duc, and S. Fischer, "Expert conciliation for multi modal person authentication systems by bayesian statistics," In Proceedings of Audio Video based Biometric Person Authentication, pp. 291-300, 1997.

[14] B. Bhanu and X. Tan, "Fingerprint indexing based on novel features of minutiae triplets," IEEE Transactions on Pattern Analysis and Machine Intelligence, Vol. 25, No. 5, pp. 616-622, 2003.

[15] R.M. Bolle, N.K. Ratha, and S. Pankanti, "Performance evaluation in 1:1 biometric engines," In Proceedings of Sinobiometrics, pp. 27-46, 2004.

[16] J.-G. Cao, J. E. Fowler, and N. H. Younan, "An image-adaptive watermark based on a redundant wavelet transform," In Proceedings of International Conference on Image Processing, Vol. 2, pp. 277-280, 2001.

[17] T. Chan and L. Vese, "Active contours without edges," IEEE Transactions on Image Processing, Vol. 10, No. 2, pp. 266-277, 2001.

[18] C. Chapel, "Fingerprinting - a manual of identification," Coward McCann, New York, 1971.

[19] Y. Chen and A.K. Jain, "Dots and incipients: extended features for partial fingerprint matching," In Proceedings of Biometric Symposium, Biometric Consortium Conference, 2007.

[20] P.H. Chen, C.J. Lin, and B. Schlkopf, "A tutorial on $\nu$-Support Vector Machines," Applied Stochastic Models in Business and Industry, Vol. 21, pp. 111-136, 2005. 
[21] Y.H. Chen and Y.Y. Yao, "Multiview intelligent data analysis based on granular computing," In Proceedings of IEEE International Conference on Granular Computing, pp. 281-286, 2006.

[22] H.G. Chew, C.C. Lim, and R.E. Bogner, "An implementation of training dual$\nu$ support vector machines," Optimization and Control with Applications, Kluwer, Edited by L. Qi, K.L. Teo and X. Yang, pp. 157182, 2005.

[23] J. Cowger, "Friction ridge skin: comparison and identification of fingerprints," Elsevier, New York, 1983.

[24] I.J. Cox, J. Ghosn, and P.N. Yianilos, "Feature-based face recognition using mixturedistance," In Proceedings of International Conference on Computer Vision and Pattern Recognition, pp. 209-216, 1996.

[25] I. Daubechies, "Ten lectures on wavelets," Society for Industrial and Applied Mathematics, 1992.

[26] J. Dezert, "Foundations for a new theory of a plausible and paradoxical reasoning," Information and Security Journal, Vol. 9, pp. 13-57, 2002.

[27] J. Dezert, and F. Smarandache, "Introduction to the fusion of quantitative and qualitative beliefs," Information and Security, Vol. 20, pp. 9-49, 2006.

[28] T.G. Dietterich, "Approximate statistical tests for comparing supervised classification learning algorithms," Neural Computation, Vol. 7, No. 10, pp. 1895-1924, 1998.

[29] Y. Dodis, L. Reyzin, and A. Smith, "Fuzzy extractors: how to generate strong keys from biometrics and other noisy data," Eurocrypt, Vol. 3027, pp. 523-540, 2004.

[30] D. Dubois and H. Prade, "On the unicity of Dempster rule of combination," International Journal of Intelligent Systems, Vol. 1, pp. 133-142, 1986.

[31] R.O. Duda, P.E. Hart, and D.G. Stork, "Pattern classification," $2^{\text {nd }}$ Edition, Wiley, 2000.

[32] P.J. Flynn, K.W. Bowyer, and P.J. Phillips, "Assessment of time dependency in face recognition: an initial study," In Proceedings of Audio and Video-Based Biometric Person Authentication, pp. 44-51, 2003. 
[33] E. Fowler, "The redundant discrete wavelet transform and additive noise," Mississippi State ERC, Mississippi State University, Technical Report MSSU-COE-ERC04-04, 2004.

[34] J.E. Fowler, "The redundant discrete wavelet transform and additive noise," IEEE Signal Processing Letters, Vol. 12, No. 9, pp. 629-632, 2005.

[35] F. Galton, "Finger prints (Reprint)," Da Capo Press, New York, 1965.

[36] B. Gunsel, U. Uludag, and A.M. Tekalp, "Robust watermarking of fingerprint images," Pattern Recognition, Vol. 35, No. 12, pp. 2739-2747, 2002.

[37] E.R. Henry, "Classification and uses of fingerprints," Routledge 85 Sons, London, 1900.

[38] A. Hicklin and R. Khanna, "The role of data quality in biometric systems," Technical Report, Mitretek Systems, 2006.

[39] T.D. Hien, Z. Nakao, and Y.-W. Chen, "Robust RDWT-ICA based information hiding," Soft Computing - A Fusion of Foundations, Methodologies and Applications, Springer, Vol. 10, No. 12 pp. 1135-1144, 2006.

[40] T.D. Hien, Z. Nakao, and Y.-W. Chen, "Robust multi-logo watermarking by RDWT and ICA," Signal Processing - Fractional calculus applications in signals and systems, Vol. 86, No. 10, pp. 2981-2993, 2006.

[41] L. Hong, Y. Wan, and A.K. Jain, "Fingerprint image enhancement: algorithm and performance evaluation," IEEE Transactions on Pattern Analysis and Machine Intelligence, Vol. 20, No. 8, pp. 777-789, 1998.

[42] L. Hua and J.E. Fowler, "A performance analysis of spread-spectrum watermarking based on redundant transforms," In Proceedings of IEEE International Conference on Multimedia and Expo, Vol. 2, pp. 553-556, 2002.

[43] A.K. Jain, R. Bolle, and S. Pankanti, "BIOMETRICS: personal identification in networked society," Kluwer Academic Publishers, 1999.

[44] A.K. Jain, Y. Chen, and M. Demirkus, "Pores and ridges: fingerprint matching using level 3 features," In Proceedings of International Conference on Pattern Recognition, Vol. 4, pp. 477-480, 2006. 
[45] A.K. Jain, Y. Chen, and M. Demirkus, "Pores and ridges: high resolution fingerprint matching using level 3 features," IEEE Transactions on Pattern Analysis and Machine Intelligence, Vol. 29, No. 1, pp. 15-27, 2007.

[46] A. Jain, J. Feng, A. Nagar, and K. Nandakumar, "On matching latent fingerprints," In Proceedings of IEEE Computer Society Workshop on Biometrics, 2008.

[47] A.K. Jain, P. Flynn, and A. Ross, "Handbook of biometrics," Springer, 2007.

[48] A.K. Jain, L. Hong, and R. Bolle, "On-line fingerprint verification," IEEE Transactions on Pattern Analysis and Machine Intelligence, Vol. 19, No. 4, pp. 302-314, 1997.

[49] A.K. Jain, K. Nandakumar, and A. Ross, "Score normalization in multimodal biometric systems," Pattern Recognition, Vol. 38, No. 12, pp. 2270-2285, 2005.

[50] A. Jain, S. Prabhakar, and L. Hong, "A multichannel approach to fingerprint classification," IEEE Transactions on Pattern Analysis and Machine Intelligence, Vol. 21, No. 4, pp. 348-359, 1999.

[51] A.K. Jain and A. Ross, "Learning user-specific parameters in a multibiometric system," In Proceedings of IEEE International Conference on Image Processing pp. 57-60, 2002.

[52] A.K. Jain, A. Ross, and S. Pankanti, "Biometrics: a tool for information security," IEEE Transactions on Information Forensics and Security, Vol. 1, No. 2, pp. 125143, 2006.

[53] A.K. Jain and U. Uludag, "Hiding biometric data," IEEE Transactions on Pattern Analysis and Machine Intelligence, Vol. 25, No. 11, pp. 1494-1498, 2003.

[54] A.K. Jain, U. Uludag, and R.L. Hsu, "Hiding a face in a fingerprint image," In Proceedings of International Conference on Pattern Recognition, pp. 756-759, 2002.

[55] X.D. Jiang, W.Y. Yau, and W. Ser, "Detecting the fingerprint minutiae by adaptive tracing the gray level ridge," Pattern Recognition, Vol. 34, No. 5, pp. 999-1013, 2001.

[56] J. Kittler, M. Hatef, R.P. Duin, and J.G. Matas, "On combining classifiers," IEEE Transactions on Pattern Analysis and Machine Intelligence, Vol. 20, No. 3, pp. 226239, 1998. 
[57] P. Kovesi, "Image features from phase congruency," Videre: A Journal of Computer Vision Research, MIT Press, Vol. 1, No. 3, pp. 1-26, 1999.

[58] K. Kryszczuk, A. Drygajlo, and P. Morier, "Extraction of level 2 and level 3 features for fragmentary fingerprints," In Proceedings of 2nd COST275 Workshop, pp. 8388, 2004.

[59] K. Kryszczuk, P. Morier, and A. Drygajlo, "Study of the distinctiveness of level 2 and level 3 features in fragmentary fingerprint comparison," In Proceedings of ECCV International Workshop on Biometric Authentication, pp. 124-133, 2004.

[60] L.I. Kuncheva, "Combining pattern classifiers," Methods and Algorithms, Wiley Interscience, 2004.

[61] D. Kundur, and D. Hatzinakos, "Digital watermarking using multiresolution wavelet decomposition," In Proceedings of International Conference on Acoustic, Speech and Signal Processing, Vol. 5, pp. 2969-2972, 1998.

[62] H.C. Lee and R.E. Gaensslen, "Advances in fingerprint technology," Elsevier, New York, 1991.

[63] E.L. Lehmann and J.P. Romano, "Testing statistical hypotheses," Springer, 2005.

[64] D. Maltoni, D. Maio, A.K. Jain, and S. Prabhakar, "Handbook of fingerprint recognition," Springer Verlag, 2003.

[65] P. Meenen, A. Ashrafi, and R. Adhami, "The utilization of a Taylor series-based transformation in fingerprint verification," Pattern Recognition Letters, Vol. 27, No. 14, pp. 1606-1618, 2006.

[66] A. Moenssens, "Fingerprint techniques," Chilton Book Company, London, 1971.

[67] K. Nandakumar, Y. Chen, S.C. Dass, and A.K. Jain, "Likelihood ratio based biometric score fusion," IEEE Transactions on Pattern Analysis and Machine Intelligence, Vol. 30, No. 2, pp. 342-347, 2008.

[68] National Institute of Standards and Technology, "Guidelines for the use of advanced authentication technology alternatives," Federal Information Processing Standards Publication, pp. 190, 1994.

[69] J. Osterberg, T. Parthasarathy, T. Raghavan, and S. Sclove, "Development of a mathematical formula for the calculation of fingerprint probabilities based on indi- 
vidual characteristic," Journal American Statistic Association, Vol. 72, pp. 772-778, 1977.

[70] T. Pavlidis, "Algorithms for graphics and image processing," Computer Science Press, 1982.

[71] F. Petitcolas, R. Anderson, and M. Kuhn, "Information hiding - a survey," In Proceedings of IEEE, Vol. 87, No. 7, pp. 1062-1078, 1999.

[72] N.K. Ratha, J.H. Connell, and R.M. Bolle, "Secure data hiding in wavelet compressed fingerprint images," In Proceedings of ACM Multimedia, pp. 127-130, 2000.

[73] O. Rioul and M. Vetterli, "Wavelets and signal processing," IEEE Signal Processing Magazine, Vol. 8, No. 4, pp. 14-38, 1991.

[74] A.R. Roddy and J.D. Stosz, "Fingerprint features - statistical analysis and system performance estimates," In Proceedings of IEEE, Vol. 85, No. 9, pp. 1390-1421, 1997.

[75] A. Ross, S. Dass, and A.K. Jain, "Fingerprint warping using ridge curve correspondences," IEEE Transactions on Pattern Analysis and Machine Intelligence, Vol. 28, No. 1, pp. 19-30, 2006.

[76] A. Ross and A.K. Jain, "Information fusion in biometrics," Pattern Recognition Letters, Vol. 24, No. 13, pp. 2115-2125, 2003.

[77] A. Ross and R. Mukherjee, "Augmenting ridge curves with minutiae triplets for fingerprint indexing," In Proceedings of SPIE Conference on Biometric Technology for Human Identification IV, 2007.

[78] A. Ross, K. Nandakumar, and A.K. Jain, "Handbook of multibiometrics," Springer Publishers, 2006.

[79] T. Roxburgh, "On the evidential value of finger prints," Sankhya: Indian Journal of Statistics, Vol. 1, pp. 189-214, 1933.

[80] K. Saviers, "Friction skin characteristics: a study and comparison of proposed standards," Garden Grove: California Police Department, 1987.

[81] B. Schlkopf, A.J. Smola, R. Williams, and P. Bartlett, "New support vector algorithms," Neural Computation, Vol. 12, pp. 1083-1121, 2000.

[82] G. Shafer, "A mathematical theory of evidence," Princeton University Press, 1976. 
[83] Y. Shi and W.C. Karl, "A real-time algorithm for the approximation of level-setbased curve evolution," IEEE Transactions on Image Processing, Vol. 17, No. 5, pp. 645-656, 2008.

[84] S.K. Singh, D.S. Chauhan, M. Vatsa, and R.Singh, "A robust skin color based face detection algorithm," Tamkang Journal of Science and Engineering, Vol. 6, No. 4, pp. 227-234, 2003.

[85] R. Singh, M. Vatsa, A. Noore and S.K. Singh, "Dempster shafer theory based classifier fusion for improved fingerprint verification performance," In Proceedings of Indian Conference on Computer Vision, Graphics and Image Processing, Vol. 4338, pp. 941-949, 2006.

[86] R. Singh, M. Vatsa, A. Noore, and S.K. Singh, "DS theory classifier fusion with update rule to minimize training time," IEICE Electronics Express, Vol. 3, No. 20, pp. 429-435, 2006.

[87] R. Singh, M. Vatsa, and A. Noore, "Intelligent biometric information fusion using support vector machine," Soft Computing in Image Processing: Recent Advances, Springer, Edited by M. Nachtegael, D. Van der Weken, E.E. Kerre, and W. Philips, Chapter 12, pp. 327-350, 2006.

[88] R. Singh, M. Vatsa, and A. Noore, "Integrated multilevel image fusion and match score fusion of visible and infrared face images for robust face recognition," Pattern Recognition - Special Issue on Multimodal Biometrics, Vol. 41, No. 3, pp. 880-893, 2008.

[89] F. Smarandache and J. Dezert, "Advances and applications of DSmT for information fusion," American Research Press, 2004.

[90] F. Smarandache, "An in-depth look at quantitative information fusion rules," Advances and Applications of DSmT for Information Fusion, American Research Press, Vol. 2, Chapter 8, pp. 205-236, 2006.

[91] P. Smets and R. Kennes, "The transferable belief model," Artificial Intelligence, Vol. 66, No. 2, pp. 191-234, 1994.

[92] P. Smets, "Decision making in a context where uncertainty is represented by belief functions," Physica-Verlag, pp. 17-61, 2002.

[93] P. Smets, "Analyzing the combination of conflicting belief functions," Information Fusion, Vol. 8, No. 4, pp. 387-412, 2007. 
[94] C. Soutar, D. Roberge, A. Stoianov, R. Gilroy, and B. Kumar, "Biometric encryption," ICSA Guide to Cryptography, McGraw-Hill, 1999.

[95] C. Stanley, "Are fingerprints a genetic marker for handedness?," Behavior Genetics, Vol. 24, No. 2, pp. 141, 1994.

[96] D.A. Stoney, "A quantitative assessment of fingerprint individuality," Ph.D. Dissertation, University of California, Berkeley, 1985.

[97] G. Strang, and T. Nguyen, "Wavelet and filter banks," Wellesly-Cambridge Press, MA, 1996.

[98] Y. Sugie and T. Kobayashi, Media-integrated biometric person recognition based on the Dempster-Shafer theory, In Proceedings of 16th International Conference on Pattern Recognition, Vol. 4, pp. 40381-40384, 2002.

[99] E. Tabassi, C. Wilson, and C. Watson, "Fingerprint image quality," NIST Research Report NISTIR 7151, 2004.

[100] Y.C. Tang and Y.-Q. Zhang, "Granular support vector machines with data cleaning for fast and accurate biomedical binary classification," In Proceedings of International Conference on Granular Computing, pp. 262-265, 2005.

[101] A. Tefas, C. Kotropoulos, and I. Pitas, "Using support vector machines to enhance the performance of elastic graph matching for frontal face authentication," IEEE Transactions on Pattern Analysis and Machine Intelligence, Vol. 23, No. 7, pp. 735746, 2001.

[102] A. Tsai, A. Yezzi Jr., and A. Willsky, "Curve evolution implementation of the Mumford-Shah functional for image segmentation, denoising, interpolation, and magnification," IEEE Transactions on Image Processing, Vol. 10, No. 8, pp. 11691186, 2001.

[103] M. Tuceryan and T. Chorzempa, "Relative sensitivity of a family of closest-point graphs in computer vision applications," Pattern Recognition, Vol. 24, No. 5, pp. 361-373, 1991.

[104] M. Turk and A. Pentland, "Eigenfaces for recognition," Journal of Cognitive Neuroscience, Vol. 3, pp. 72-86, 1991.

[105] B. Ulery, A. R. Hicklin, C. Watson, W. Fellner, and P. Hallinan, "Studies of biometric fusion," NIST Technical Report IR 7346, 2006. 
[106] U. Uludag, S. Pankanti, S. Prabhakar, and A.K. Jain, "Biometric cryptosystems: issues and challenges," In Proceedings of IEEE, Vol. 92, No. 6, pp. 948-960, 2004.

[107] V. Vapnik, "The nature of statistical learning theory," Springer, 1995.

[108] V. Vapnik, S.E. Golowich, and A. Smola, "Support vector method for function approximation, regression estimation and signal processing," Advances in Neural Information Processing Systems, Vol. 9, pp. 281-287, 1997.

[109] M. Vatsa, R. Singh, P. Mitra, and A. Noore, "Comparing robustness of watermarking algorithms on biometrics data," In Proceedings of Workshop on Biometric Challenges from Theory to Practice - ICPR Workshop, pp. 5-8, 2004.

[110] K. Veeramachaneni, L.A. Osadciw, and P.K. Vasrhney, "An adaptive multimodal biometric management algorithm," IEEE Transactions on Systems Man and Cybernetics, Part C, Vol. 35, No. 3, pp. 344-356, 2005.

[111] F. Voorbraak, "On the justification of Dempsters rule of combination," Artificial Intelligence, Vol. 48, pp. 171-197, 1991.

[112] K. Woods, W.P. Kegelmeyer, and K.W. Bowyer, "Combination of multiple classifiers using local accuracy estimates," IEEE Transactions on Pattern Analysis and Machine Intelligence, Vol. 19, No. 4, pp. 405-410, 1997.

[113] Working draft of CDEFFS: the ANSI/ NIST committee to define an extended fingerprint feature set. Available at http://fingerprint.nist.gov/standard/cdeffs/index.html, 2008.

[114] R.R. Yager, J. Kacprzyk, and M. Fedrizzi, "Advances in the Dempster-Shafer theory of evidence," Wiley, 1994.

[115] Y.Y. Yao, "Perspectives of granular computing," In Proceedings of IEEE International Conference on Granular Computing, Vol. 1, pp. 85-90, 2005.

[116] L. Zadeh, "On the validity of Dempsters rule of combination," University of California, Berkeley, Memo M 79/24, 1979.

[117] L. Zadeh, "Review of mathematical theory of evidence by Glenn Shafer," AI Magazine, Vol. 5, No. 3, pp. 81-83, 1984.

[118] L. Zadeh, "A simple view of the Dempster-Shafer theory of evidence and its implication for the rule of combination," AI Magazine, Vol. 7, No. 2, 85-90, 1986. 
[119] Z. Zivkovic and F.Van Der Heijden, Recursive unsupervised learning of finite mixture models, IEEE Transactions on Pattern Analysis and Machine Intelligence, Vol. 26, No. 5, pp. 651-656, 2004. 